\title{
Needle-free Interstitial Fluid Acquisition Using a Lorentz-Force Actuated Jet Injector
}

by

\author{
Jean H. Chang
}

S.B., Massachusetts Institute of Technology (2008)

S.M., Massachusetts Institute of Technology (2010)

Submitted to the Department of Mechanical Engineering in partial fulfillment of the requirements for the degree of

Doctor of Philosophy in Mechanical Engineering at the

\section{MASSACHUSETTS INSTITUTE OF TECHNOLOGY}

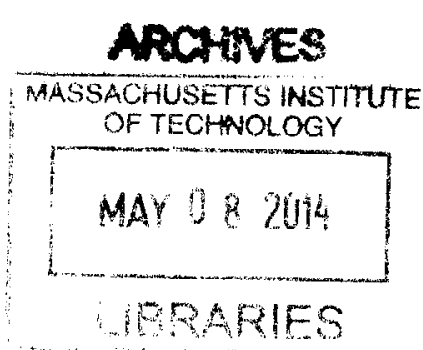

February 2014

(C) Massachusetts Institute of Technology 2014. All rights reserved.

Author

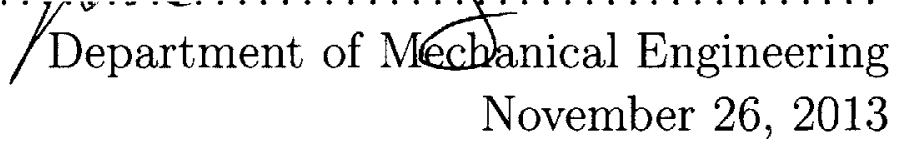

Certified by .........

Ian W. Hunter

Hatsopoulos Professor of Mechanical Engineering Thesis Supervisor

Accepted by ................... 


\title{
Needle-free Interstitial Fluid Acquisition Using a
}

\section{Lorentz-Force Actuated Jet Injector}

\author{
by \\ Jean H. Chang \\ Submitted to the Department of Mechanical Engineering \\ on November 26, 2013, in partial fulfillment of the \\ requirements for the degree of \\ Doctor of Philosophy in Mechanical Engineering
}

\begin{abstract}
Interstitial fluid (ISF) provides information on a patient's health as it contains regulatory molecules that are correlated with disease-related processes. However, current ISF acquisition techniques can be slow, resulting in patient discomfort and erroneous measurements. This thesis presents a fast $(<4 \mathrm{~s})$, minimally-invasive, needle-free technique of extracting ISF samples using a Lorentz-force actuated jet injector. The jet injector is used to first inject a small volume of physiological saline to breach the skin, and the actuator is subsequently back-driven to create a vacuum in the ampoule and collect a sample that contains a mixture of ISF and injectate.

The scope of this thesis is twofold: the first part aims to investigate the effect of jet injection on tissue, while the second part focuses on the development of the novel ISF acquisition method. A micro-CT imaging study identifies the magnitudes of injected jet speed that will influence injectate delivery to specific skin layers. A histology study highlights the differences in tissue damage between needle injections and jet injections. A new tool for quantifying the jet dynamics in tissue - a high-speed $\mathrm{X}$-ray imaging system-is built and characterized. The system, which has a capture rate of up to 2,000 fps, is used to visualize jet injections into tissue in real-time, and for the first time measurements are made of the fluid speed in tissue.

To develop the jet injector for ISF acquisition, a finite element model that describes the effect of different injection and extraction parameters on the ISF acquisition process is developed. The model is used to explain the trends seen in experimental work on post-mortem tissue, and the lessons learned from both the model and experimentation are used to identify the parameters for a live animal study. The feasibility of the acquisition process is successfully demonstrated on live rats; the process is revealed to extract samples that have been diluted by a factor of 111-125.
\end{abstract}

Thesis Supervisor: Ian W. Hunter

Title: Hatsopoulos Professor of Mechanical Engineering 


\section{Acknowledgments}

First and foremost, I would like to thank my advisor, Professor Ian Hunter. I am extremely lucky to have worked in the BioInstrumentation Lab. Ian has always been incredibly supportive, making sure that his students had access to all the resources that we needed for our projects. I am lucky to have had an advisor who has encouraged me to explore the aspects of my project that I found interesting, and who has pushed me to constantly learn new skills. I have learned so much over the past six years.

I would like to thank Sanofi-Aventis for the financial support for this thesis.

I would also like to thank my committee members, Professor Linda Griffith and Professor Rohit Karnik. I appreciate the words of advice given to me during the course of this thesis.

Dr. Cathy Hogan has taught me so much during my graduate career, both in the professional sense and in the personal sense. She has become a great friend, someone to joke around with, gossip with, and share my concerns. I will miss you.

I would like to thank Professor Evelyn Wang, my mentor through Graduate Women at MIT. She has been such an amazing role model and has provided great advice as well as thoughtful words of encouragement to me for the last couple of years. I would also like to thank the other professors in the mechanical engineering department who have indirectly served as my mentors during my time at MIT. They have encouraged me to constantly challenge myself while at the same time making sure that I was enjoying it.

Kate Melvin, thank you so much for all the work that you do in keeping the lab running. I could always count on her to have an amazingly positive attitude. Leslie Regan, Joan Kravit, and Una Sheehan also deserve a huge thank you for everything that they do to keep the department running smoothly.

Throughout my graduate career I have had the pleasure of working with some of the brightest people I have ever met. Dr. Priam Pillai taught me so much when I first entered the lab, and mentored me until I felt confident enough to stand on my own. Dr. Brian Hemond, Dr. Bryan Ruddy, and Dr. Adam Wahab all provided 
genius advice to me during my $\mathrm{PhD}$. Ellen and Eli, I have always felt a special bond with you guys because we all started together. To the other BioInstrumentation lab members, Ashley Brown, Alison Cloutier, Nick Demas, Kerri Keng, Dr. Walker Inman, John Liu, Ashin Modak, Mike Nawrot, Geehoon Park, Miguel Saez, Span, and Jamie White, thank you so much for always being there to bounce ideas and chat when I needed a distraction.

I was fortunate to have been given the opportunity to spend a few months working at our collaborator's lab in New Zealand. This was made possible by of course my generous advisor Ian, and also by Dr. Peter Hunter and Dr. Andrew Taberner. The members of the Auckland Bioengineering Institute were incredibly welcoming to me when I arrived. Dr. Jessica Jor and Adam Reeve spent time training me on new equipment, and Rhys Williams spent a good amount of time helping me set up my jet injector. Alex Anderson, Ming Cheuk, Mark Finch, Nikini Gamage, Prasad Babarenda Gamage, Callum Johnston, Tom Lintern, Matt Parker, and Paul Roberts also made me feel so welcome and made sure that I was enjoying my visit to New Zealand. I would also like to thank the MISTI Global Seed Fund for the financial support to travel to New Zealand.

I can confidently say that my friends played a major role in me finishing my $\mathrm{PhD}$. They have always been a wonderful distraction from the rigors of graduate school. I am lucky to have such amazing friends. When I look back at my time in graduate school, I know that I will remember most the incredible times I had with my friends.

Finally, I would like to thank my family who has given me so much love and has been so supportive of me in everything that I have done. My parents know exactly how much to push me, and at the end of the day they just want me to be happy. Their number one piece of advice that they gave me while I was growing up was that I should always make sure that I was happy doing what I was doing. I am lucky to have such a great relationship with my sister. Even though we have quite different personalities, we still manage to have that "sister thing," where we can read each other's minds. And I am proud of my little brother, who I have watched become an adult these past few years. 


\section{Contents}

1 Introduction $\quad 21$

1.1 Motivation . . . . . . . . . . . . . . . . . . 21

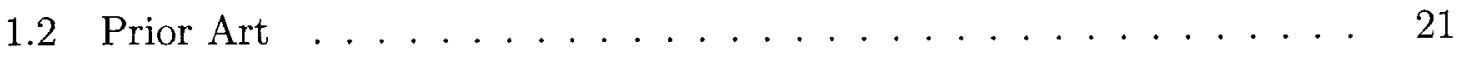

1.3 Fluid Sample Acquisition using a Needle-free Jet Injector . . . . . . . 23

1.4 Chapter Descriptions . . . . . . . . . . . . . . . . 26

2 Device Development $\quad 27$

2.1 Device description . . . . . . . . . . . . . . . . . . . 27

2.1.1 Control System Hardware . . . . . . . . . . . . . . 29

2.1 .2 Control System Software . . . . . . . . . . . . . . . . . 29

2.2 Summary . . . . . . . . . . . . . . . . . 36

$\begin{array}{lll}3 & \text { Injection Studies } & 37\end{array}$

3.1 Background: Skin Layers . . . . . . . . . . . . . . . . . . . . . 39

3.1 .1 Microstructure . . . . . . . . . . . . . . 40

3.2 Effect of Injection Parameters on Injection Depth . . . . . . . . . 44

3.2.1 Optimal Delivery Target: the Dermis . . . . . . . . . . 44

3.2 .2 Importance of contact force $\ldots \ldots \ldots \ldots . \ldots . \ldots 45$

3.2 .3 Experimental Methods . . . . . . . . . . . . . . . . 46

3.2.4 Results and Conclusions . . . . . . . . . . . . . 46

3.3 X-ray Microtomography Studies of Jet Injections _ . . . . . . . . 49

3.3.1 Experimental Methods . . . . . . . . . . . . . . . . . 49

3.3 .2 Results . . . . . . . . . . . . . . . . . . . . 50 
3.4 Histological examination of tissue damage due to jet injections . . . 58

3.4.1 Experimental Methods . . . . . . . . . . . . 58

3.4 .2 Results ....................... 59

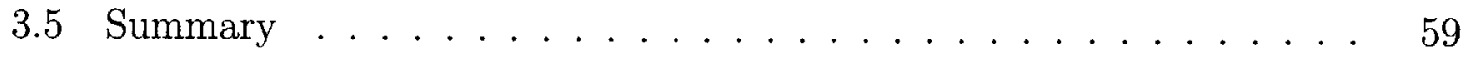

4 High-speed X-ray imaging studies $\quad 63$

4.1 Device development .................... 63

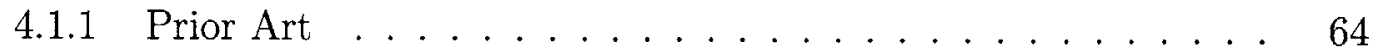

4.1.2 Functional requirements ............... 65

4.1.3 Component selection ............... 66

4.1 .4 Characterization ................... 68

4.1.5 Future improvements ................ 70

4.2 X-ray imaging of needle injections . . . . . . . . . . . . . 71

4.3 High speed X-ray imaging of jet injections . . . . . . . . . . . 73

4.4 Summary ............................ 78

5 Modeling of Interstitial Fluid Acquisition Method $\quad 79$

5.1 Model setup and assumptions . . . . . . . . . . . . . . 79

5.1 .1 Darcy's Law . . . . . . . . . . . . . . . . 80

5.1.2 Resistance to Dermal Interstitial Flow . . . . . . . . . . 81

5.1.3 Change in Tissue Permeability due to Swelling . . . . . . . 86

5.1 .4 Dermis model . . . . . . . . . . . . . . 90 90

5.2 Modeling of Vacuum Acquisition Methods . . . . . . . . . . . . 92

5.2 .1 Model setup . . . . . . . . . . . . . . . 92

5.2 .2 Results............................. 94

5.3 Modeling of JI ISF Acquisition Method . . . . . . . . . . . . 95

5.3 .1 Modeling Injection . . . . . . . . . . . . . . 96

5.3.2 Simulations of the Effect of Injection Parameters . . . . . . 98

5.3.3 Simulations of the Effect of Extraction Parameters . . . . . 101

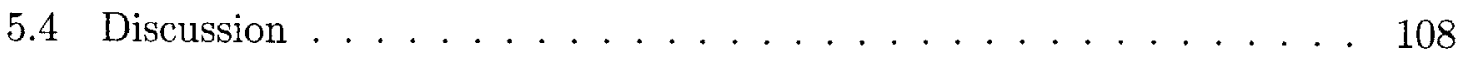


6 Extraction studies $\quad 111$

6.1 Experiments on Post-Mortem Tissue: Porcine . . . . . . . . . . . . 111

6.1.1 Experimental Methods .............. 111

6.1 .2 Dependence on Injection Parameters . . . . . . . . . . 112

6.1.3 Dependence on Extraction Parameters . . . . . . . . . . 116

6.2 Lessons learned from modeling and experimentation . . . . . . . . 120

6.2.1 Experiments with benchtop device on post-mortem rat skin . 120

6.2 .2 Experiments with Handheld Device . . . . . . . . . . . . 121

6.3 Live animal studies: rat . . . . . . . . . . . . . . 123

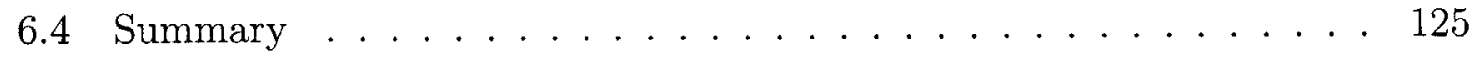

7 Conclusions $\quad 127$

7.1 Suggestions for Future Work . . . . . . . . . . . . . . 128

7.1.1 Effect of jet injection on tissue . . . . . . . . . 128

7.1 .2 ISF acqusition . . . . . . . . . . . . . . 128 


\section{List of Figures}

1-1 Interstitial fluid delivers nutrients to as well as removes waste products from cells. Figure from $[1] \ldots \ldots \ldots 22$

1-2 3D rendering of Lorenz force actuated needle-free injector. Figure courtesy of Dr. Andrew Taberner. . . . . . . . . . . . . . . . .

1-3 Schematic of extraction process. (a) Physiological saline is injected into the skin. (b) Injectate mixes with ISF. (c) Piston direction is reversed. The vacuum created in the ampoule along with the pressure increase in the tissue due to the injection allows for the sample to flow into the ampoule. . . . . . . . . . . . . . .

2-1 Jet injector benchtop device. . . . . . . . . . . . . . . 28

2-2 Flow chart illustrating the state machine for the new software. . . . 30

2-3 Magnitude and phase data used to fit a third order linear model to describe the coil and ampoule dynamics. . . . . . . . . . . 31

2-4 The FPGA first builds the acceleration waveform (top left) and then generates the corresponding velocity (top right) and position (bottom left) waveforms by integration. . . . . . . . . . . . . . 33

2-5 Results from a voltage-velocity calibration. . . . . . . . . . . 34

2-6 The injection controller used a velocity-voltage feedforward controller along with a PID feedback controller. . . . . . . . . . . 35

2-7 Average ejected volume errors. . . . . . . . . . . . . 35

2-8 Average tracking errors. . . . . . . . . . . . 35

3-1 Two-phase waveform used for jet injections. . . . . . . . . 38 
3-2 The structure of skin is layered and consists of the epidermis, the dermis, the subcutaneous fat, and the muscle. Figure modified from [2].

3-3 Scanning electron microscopy images of the fibrous tissue in the dermis. The denser papillary layer makes up approximately $10 \%$ of the dermal thickness, while the coarser reticular layer makes up the remaining $90 \%$ of the dermis. Figure adapted from [3] . . . . . . . . . .

3-4 The stress-strain curve of a collagen matrix is ' $\mathrm{J}$ '-shaped, with three different zones. Figure from $[4]$. . . . . . . . . . . . . . . .

3-5 Histological section of porcine adipose tissue at two different magnifications. Left image shows septa and right image shows the reinforced basement membrane that surrounds each adipocyte. Figure from [5].

3-6 The structure of skeletal muscle. . . . . . . . . . . . .

3-7 The dispersion depths measured for various values of $v_{j e t}$ and contact force. For this set of experiments, $v_{\text {follow }}=50 \mathrm{~m} / \mathrm{s}$ and $\mathrm{Vol}=100 \mu \mathrm{L}$. The error bars represent standard errors. . . . . . . . . . . . . .

3-8 The dispersion depths measured for various values of Vol. For this set of experiments, $v_{\text {jet }}=150 \mathrm{~m} / \mathrm{s}, t_{\text {jet }}=2 \mathrm{~ms}$, and $v_{\text {follow }}=50 \mathrm{~m} / \mathrm{s}$. The error bars represent standard errors. . . . . . . . . . . . . . . .

3-9 The dispersion depths measured for various values of Vol. For this set of experiments, $v_{j e t}=175 \mathrm{~m} / \mathrm{s}, t_{\text {jet }}=2 \mathrm{~ms}$, and $v_{\text {follow }}=50 \mathrm{~m} / \mathrm{s}$. The error bars represent standard errors. . . . . . . . . . . . . .

3-10 Micro-CT images (two-dimensional) of the dispersion pattern for a $100 \mu \mathrm{L}$ injection into the subcutaneous fat layer using a 27 gauge (410 $\mu \mathrm{m}$ nominal OD) needle. . . . . . . . . . . . . . . .

3-11 Micro-CT images (two-dimensional) of the dispersion pattern for a $100 \mu \mathrm{L}$ injection into the subcutaneous fat layer using the JI. . . . .

3-12 3D reconstructed volume of a jet injected tissue sample. There was the consistent appearance of 'streams' of injectate in the subcutaneous fat layer. . . . . . . . . . . . . . . . . . 
3-13 Section from a jet injected tissue sample $\left(v_{j e t}=175 \mathrm{~m} / \mathrm{s}, t_{j e t}=2 \mathrm{~ms}\right.$, $v_{\text {follow }}=25 \mathrm{~m} / \mathrm{s}, \mathrm{Vol}=100 \mu \mathrm{L}$ ) stained with picrosirius red. . . . . . 54

3-14 Top-down view of 3D reconstructed micro-CT scan of a porcine tissue sample jet injected with $\mathrm{Vol}=100 \mu \mathrm{L}$, at $v_{j e t}=175 \mathrm{~m} / \mathrm{s}, t_{j e t}=2 \mathrm{~ms}$, and $v_{\text {follow }}=25 \mathrm{~m} / \mathrm{s}$. The location of the section shown in Figure 3-13 is indicated. The yellow arrow notes the 'stream.' . . . . . . . . .

3-15 3D reconstructed volume of a micro-CT scan of a $100 \mu \mathrm{L}$ intramuscular needle injection. A 27 gauge (410 $\mu \mathrm{m}$ nominal OD) needle was used. The contrast was injected after the needle was retracted $2 \mathrm{~mm}$. . . . .

3-16 3D reconstructed volume of a micro-CT scan of a $100 \mu \mathrm{L}$ intramuscular needle injection. A 27 gauge $(410 \mu \mathrm{m}$ nominal OD) needle was used. In this case, the needle was not retracted before the contrast was injected. 56

3-17 3D reconstructed volume of a micro-CT scan of an intramuscular jet injection, where $v_{\text {follow }}=25 \mathrm{~m} / \mathrm{s}$. The white arrow denotes the injection

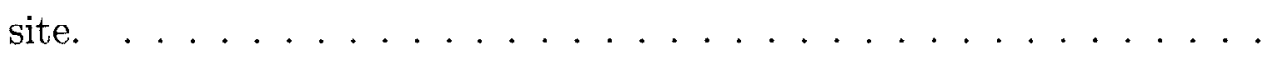

3-18 3D reconstructed volume of a micro-CT scan of an intramuscular jet injection, where $v_{\text {follow }}=63 \mathrm{~m} / \mathrm{s}$. The white arrow denotes the injection site. . . . . . . . . . . . . . . . .

3-19 Percentage of injectate delivered to each skin layer for different values of $v_{\text {follow. }}$. Injections were performed with $v_{\text {jet }}=175 \mathrm{~m} / \mathrm{s}, t_{\text {jet }}=2 \mathrm{~ms}$, and $V o l=100 \mu \mathrm{L}$. In these cases, the injectate did not reach the muscle layer. . . . . . . . . . . . . . . . . . . .

3-20 Percentage of injectate delivered to each skin layer for different values of $v_{\text {follow }}$ for intramuscular injections. Injections were performed with $v_{j e t}=175 \mathrm{~m} / \mathrm{s}, t_{j e t}=2 \mathrm{~ms}$, and $V o l=100 \mu \mathrm{L} \ldots \ldots$.

3-21 Micrographs of $20 \mu \mathrm{m}$ thick cryotomed tissue sections stained with picrosirius red. The sections show the damage resulting from the puncture of the tissue by the jet. The tissue sample was jet injected with the following parameters: $v_{\text {jet }}=175 \mathrm{~m} / \mathrm{s}, t_{\text {jet }}=2 \mathrm{~ms}, v_{\text {follow }}=50 \mathrm{~m} / \mathrm{s}$, and $V o l=100 \mu \mathrm{L} . \ldots \ldots \ldots \ldots$ 
3-22 Micrographs of $20 \mu \mathrm{m}$ thick cryotomed tissue sections stained with picrosirius red. The sections show the damage resulting from the dispersion of the injectate volume into the tissue, from injections performed with a 27 gauge $(410 \mu \mathrm{m}$ nominal OD) needle and the JI. . . . . . . 61

4-1 Previous X-ray imaging setup. Photo by Dr. Adam Wahab. . . . . 65

4-2 The high-speed X-ray imaging setup. The light-blocking tube is not shown . . . . . . . . . . . . . . . . . . . . .

4-3 Geometric unsharpness, defined by the size of the penumbra $U g$, is dependent on the X-ray source focal-spot size, the distance from the $\mathrm{x}$-ray source to the front surface of the object, and the distance from the object to the detector. Figure from $[6] \ldots \ldots \ldots$. . . . . .

4-4 The needle injection system used to inject a set volume of fluid into post-mortem porcine skin. . . . . . . . . . . . . . .

4-5 Frames from X-ray video taken of a $100 \mu \mathrm{L}$ intramuscular needle injection into post-mortem porcine skin. The injections were performed without retracting the needle. . . . . . . . . . . . . . . .

4-6 Frames from X-ray video taken of a $100 \mu \mathrm{L}$ intramuscular needle injection into post-mortem porcine skin. The injection was performed after retracting the needle approximately $2 \mathrm{~mm} \ldots \ldots \ldots \ldots$

4-7 High-speed X-ray video was taken of a needle-free jet injection at a frame rate of $2,000 \mathrm{fps}$ and an exposure time of $490 \mu \mathrm{s}$. . . . . . . .

5-1 The permeability changes by several orders of magnitude with a change in porosity.

5-2 Box-like pore 'element' used to model the effect of swelling on dermis permeability.

5-3 Calculated dermal permeability, $k_{I S}$, as a function of volumetric strain. 90

5-4 When fluid flows through porous media, the fluid must flow around solid particles, resulting in local variations in pore fluid velocities and additional mixing. . . . . . . . . . . . . . . . . . . . . . 91 
5-5 The geometry and boundary conditions used to simulate a vacuum-only acquisition. An axisymmetric geometry with side $e$ as the centerline was used. SM stands for the solid mechanics interface, ST stands for the solute transport module, and FD stands for the fluid dynamics interface. . . . . . . . . . . . . . . . .

5-6 Fluid velocity at the center of the orifice when a vacuum of $-66.1 \mathrm{kPa}$ was applied at the orifice. . . . . . . . . . . . . . . . 94

5-7 Tissue permeability during a simple vacuum acquisition method. Color bar represents tissue permeability in $\left[\mathrm{m}^{2}\right] \ldots \ldots \ldots \ldots$

5-8 Pressure distribution in the tissue during a vacuum acquisition (color bar represents pressure in $[\mathrm{Pa}])$. The arrows depict fluid velocitics (direction and magnitude) . . . . . . . . . . . . . . .

5-9 The geometry and boundary conditions used to simulate an injection prior to extraction. An axisymmetric geometry with side $f$ as the centerline was used. SM stands for the solid mechanics interface, ST stands for the solute transport module, and FD stands for the fluid dynamics interface. . . . . . . . . . . . . .

5-10 Pressure distribution in the tissue during an acquisition, after being injected with $25 \mu \mathrm{L}$ of fluid (color bar represents pressure in [Pa]). The arrows depict fluid velocities (direction and magnitude). . . . . .

5-11 Maximum pressure in the tissue for an injected volume of $25 \mu \mathrm{L}$ (injection pressure of $29 \mathrm{MPa}$, injection time of $1.8 \mathrm{~s}$ ). . . . . . 100

5-12 Depth of the maximum pressure in the tissue for an injected volume of $25 \mu \mathrm{L}$ (injection pressure of $29 \mathrm{MPa}$, injection time of $1.8 \mathrm{~s}$ ). . . 100

5-13 Results showing the effect of injection velocity on extracted volume and concentration, for an injected volume of $50 \mu \mathrm{L} \ldots \ldots \ldots . . .102$

5-14 Results showing the effect of injection volume ( $\mathrm{Vol}$ ) on extracted volume and concentration. . . . . . . . . . . . . . . . . 103

5-15 As the injected volume increases, the concentration of fluid at the orifice decreases. . . . . . . . . . . . . . . . . . . . . . . . 104 
5-16 Results showing the effect of wait time $\left(t_{\text {wait }}\right)$ on extracted volume and concentration. . . . . . . . . . . . . 106

5-17 The effect of extraction speed $\left(v_{\text {piston }}\right)$ on extracted volume and concentration. ..................... 107

5-18 Tissue permeability at the orifice as a function of time and extraction

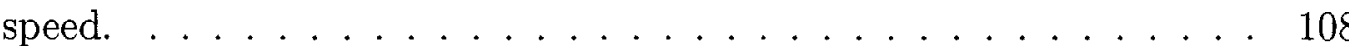

6-1 (a) Undiluted ISF obtained by centrifuging fluid out of a tissue sample; (b) Surface contamination sample collected by placing $20 \mu \mathrm{L}$ of sterile saline on the skin surface; (c) Extracted sample was a mixture of the injectate and ISF . . . . . . . . . . . . . . . . . 112

6-2 Extraction waveforms tested. . . . . . . . . . . . . . . 113

6-3 Results of trials testing different injection parameters. (a) Extracted protein and glucose concentrations (normalized by concentration in undiluted ISF) of samples. (b) Extracted volumes. Error bars in both plots represent standard errors. . . . . . . . . . . . . . . . . . 115

6-4 Results of trials testing different single-phase extraction parameters. (a) Extracted protein and glucose concentrations (normalized by concentration in undiluted ISF) of samples. (b) Extracted volumes. Error bars in both plots represent standard errors. . . . . . . . . . . . . . 117

6-5 Results of trials testing extraction waveform shapes. (a) Extracted protein and glucose concentrations (normalized by concentration in undiluted ISF) of samples. (b) Extracted volumes. Error bars in both plots represent standard errors. . . . . . . . . . . . . . . . . . . . 119

6-6 Results of experiments testing different values of $v_{1}$ on post-mortem rat skin. (a) Extracted glucose concentrations (normalized by concentration in undiluted ISF). (b) Extracted volumes. Error bars in both plots represent standard errors. . . . . . . . . . . . . . . . . . . . 122

6-7 Handheld version of JI for live animal testing. . . . . . . . . . . 123

6-8 Interstitial fluid acquisition sites. . . . . . . . . . . . . . . 124 
6-9 Appearance of rat skin after interstitial fluid acquisition. . . . . . . . 124

6-10 Average dimensions of the blisters formed due to ISF acquisition. . . 125 


\section{List of Tables}

4.1 Resolution of high-speed X-ray imaging system . . . . . . . . . 70

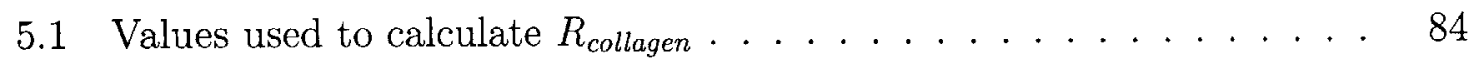

5.2 Values used to calculate $\kappa_{P} \ldots \ldots \ldots$. . . . . . . . 85

6.1 Injection parameters . . . . . . . . . . . . . . 114

6.2 Single-phase extraction parameters . . . . . . . . . . . . 117

6.3 Extraction waveform parameters . . . . . . . . . . 118

6.4 Results from live rat study (samples extracted from the abdomen) . . 125 


\section{Chapter 1}

\section{Introduction}

\subsection{Motivation}

Interstitial fluid (ISF) is the fluid that bathes cells and allows for the exchange of materials between cells and capillaries (Figure 1-1). It contains regulatory molecules such as ions, glucose, amino acids, proteins, and other nutrients which can provide information about the health of the subject. In particular, it has been shown that glucose levels in dermal interstitial fluid are highly correlated with blood glucose levels [7]. Studies have also shown that the presence of certain signaling substances, metabolized products, and proteins are correlated with disease-related processes $[8,9]$. For example, there has been recent interest in proteomic characterization of tumor interstitial fluid, the fluid that perfuses the tumor microenvironment. Studies on the protein composition of tumor interstitial fluid could lead to a new resource for diagnostic biomarker discovery and the ability to identify more selective targets for cancer treatments [10]. Determining the composition of an ISF sample can thus aid in early disease detection and in the evaluation of treatment efficacy.

\subsection{Prior Art}

There is currently a lack of minimally invasive ISF acquisition devices that are able to quickly collect samples (in a matter of seconds). ISF acquisition devices are slow 


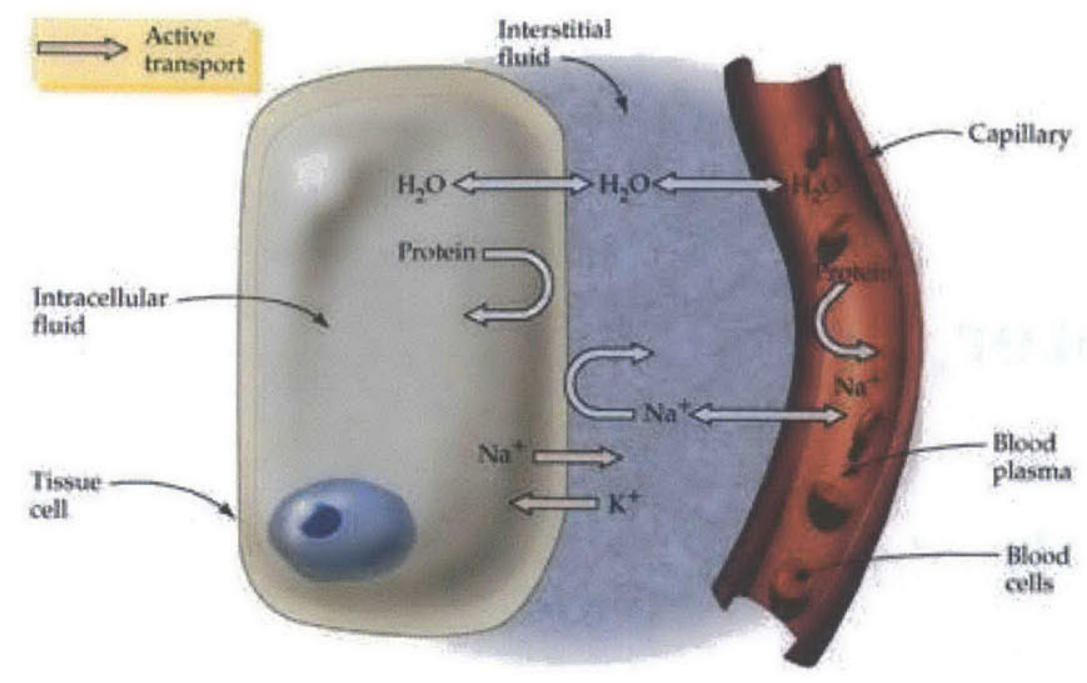

Figure 1-1: Interstitial fluid delivers nutrients to as well as removes waste products from cells. Figure from [1].

(require vacuum application for tens of minutes) due to the low permeability of the tissue matrix. Slow acquisition methods not only can cause more pain and discomfort for the patient, but also suffer from erroneous measurements since the application of a vacuum for several minutes may increase net capillary filtration resulting in a falsely low interstitial fluid concentration [11].

Additionally, while improvements have been made in the past 15 years on older methods of sample acquisition (e.g. tissue excision [11], implantable wicks [12], or suction blister devices [13]), most devices are still invasive and involve the use of needles. The drawbacks of needle-based methods include needle phobia, accidental needle-stick injury, and needle reuse in developing countries [14]. Typical ISF extraction devices involve breaching the skin using a lancet or laser followed by application of a vacuum for at least 10-20 minutes to collect the sample [15, 16].

Newer devices such as microneedle arrays which employ capillary action to draw ISF claim to reduce the amount of pain as the microneedles have small radii (tens of $\mu \mathrm{m}$ ) and penetrate shallow depths (less than $1.5 \mathrm{~mm}$ ). Relying on capillary action alone, however, results in very long acquisition times (20-30 $\mathrm{min})$ [17]. Some researchers have combined microneedle arrays with the application of a vacuum to speed up the process, but the user must still wait at least 10 minutes to collect a 1-10 
$\mu \mathrm{L}$ sample [18]. ISF acquisition using microneedle arrays is therefore a slow process, and can also suffer from other problems such as tissue clogging [19] and inflammatory responses at the insertion site [17].

A recent advancement in ISF acquisition is the development of sonophoretic devices. Sonophoretic devices apply low frequency ultrasound to the skin to induce the formation of cavitation bubbles. The formation and collapse of cavitation bub-

bles increases skin permeability by increasing skin porosity [20], and when combined with the application of a vacuum for 5-15 minutes results in a noninvasive extraction method. Mitragotri et al. demonstrated the enhanced extraction of glucose, protein, and calcium using sonophoresis when compared to passive diffusion methods $[21,22]$. Sonophoresis eliminates the requirement to breach the stratum corneum, but the method is only capable of obtaining ISF from shallow depths (epidermal ISF) [23], and is unable to collect larger items such as cells.

A promising class of interstitial fluid monitors are implantable devices, which continuously monitor analyte levels $[24,25]$. However, such devices have also been known to yield inaccurate measurements due to inflammatory responses at the implantation site [26].

\subsection{Fluid Sample Acquisition using a Needle-free Jet Injector}

The jet injector (JI) developed at the BioInstrumentation Lab is a novel Lorentz-force actuated needle-free device that allows for precise control over penetration depth and injected volume (Figure 1-2). A software-controlled linear Lorentz-force motor propels the piston of a drug ampoule, ejecting the fluid contained in the ampoule out of a small ( $\sim 200 \mu \mathrm{m}$ diameter) orifice at a pressure high enough to penetrate tissue. The jet injector offers advantages over other commercially available needle-free injectors because the Lorentz force actuator allows for real-time servo control of parameters such as jet velocity and injection volume. Commercially available needle-free injectors 
are driven by pressure sources generated by a spring mechanism or compressed gas, which do not allow for closed-loop control.

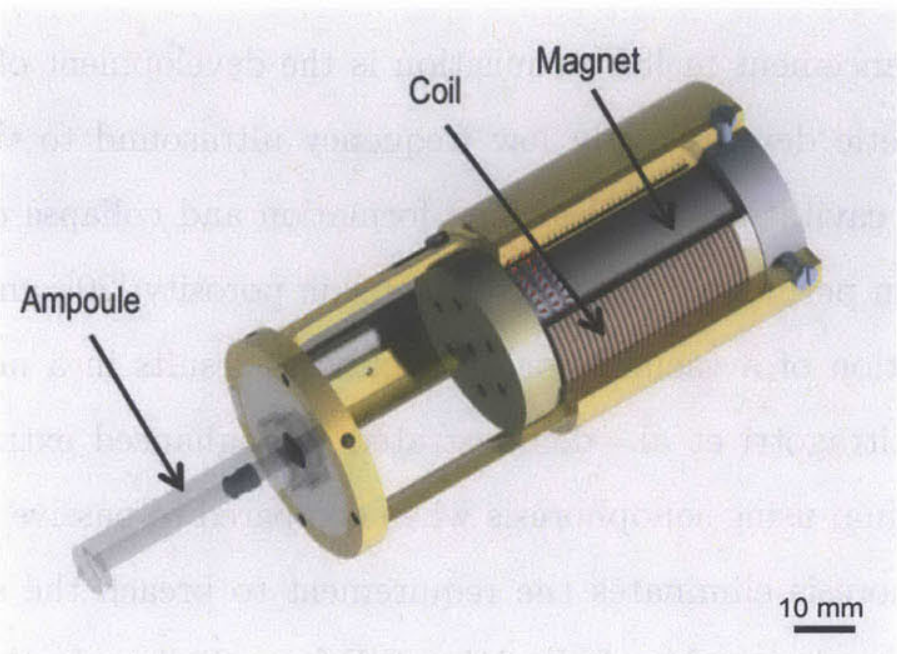

Figure 1-2: 3D rendering of Lorenz force actuated needle-free injector. Figure courtesy of Dr. Andrew Taberner.

The work presented in this thesis seeks to utilize the bi-directionality of the jet injector's Lorentz-force motor to develop a minimally invasive needle-free technique for obtaining ISF samples. The jet injector will be used to deliver a high-pressure jet of physiological saline to puncture the tissue (Figure 1-3a). The high speed of the injectate allows for mixing with the interstitial fluid in the tissue (Figure 1$3 \mathrm{~b})$. The bi-directionality of the Lorentz-force actuator is then utilized to reverse the piston direction, creating a vacuum inside the ampoule. The injection causes an instantaneous burst of pressure within the tissue, and if the piston is reversed before the injectate fully disperses into the surrounding tissue, the pressure difference allows for a mixture of the injectate and interstitial fluid to flow into the ampoule (Figure 1-3c) in a much shorter period of time than with the simple application of a vacuum.

The lack of needles in this technique can result in a smaller puncture hole (i.e. less tissue damage) at the treatment site (a 27 gauge needle is $410 \mu \mathrm{m}$ in diameter, while the fluid jet created by the jet injector can be designed to be 50-200 $\mu \mathrm{m}$ in diameter). More notably, this technique offers advantages over other ISF acquisition devices in that it is able to obtain a fluid sample in a fraction of the time that other 

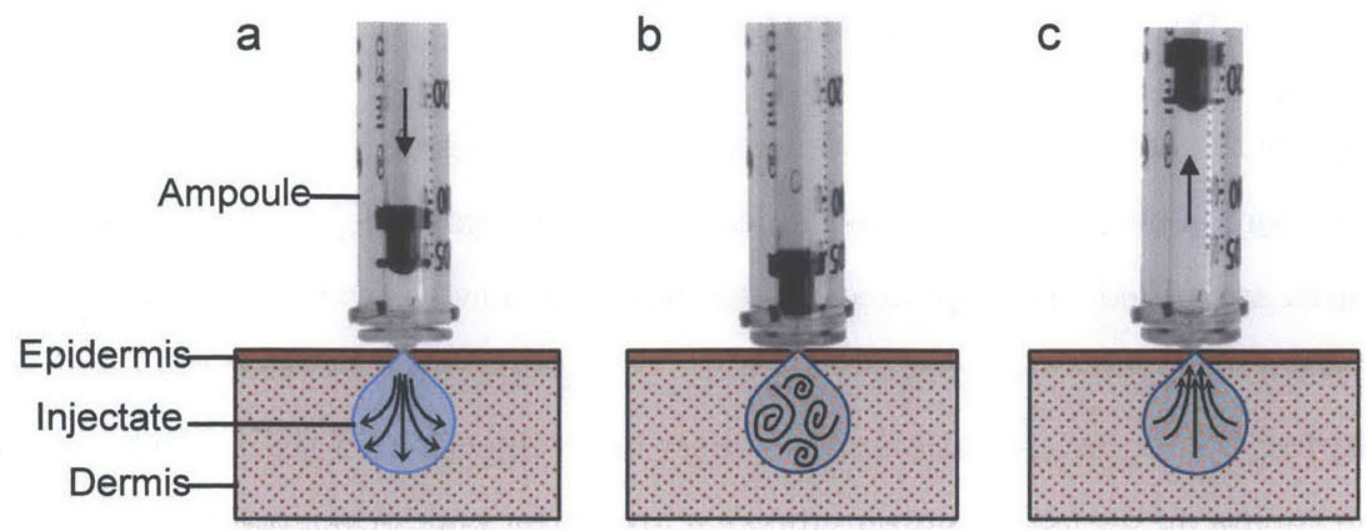

Figure 1-3: Schematic of extraction process. (a) Physiological saline is injected into the skin. (b) Injectate mixes with ISF. (c) Piston direction is reversed. The vacuum created in the ampoule along with the pressure increase in the tissue due to the injection allows for the sample to flow into the ampoule.

devices require, which can lead to less pain and discomfort and increased patient compliance. The short duration of the acquisition process allows for a measurement that is representative of a moment in time, rather than averaged over tens of minutes. Since the time constant of diffusion is on the order of minutes, there is not enough time for the body to equalize the imbalance caused by the injection of saline, nor is there enough time to produce an inflammatory response.

However, to develop the jet injector for interstitial fluid acquisition, one must first understand the effect of the injection process on tissue. The majority of work thus far have been on gel analogs, and we are just beginning to see studies on the effect of jet injections on tissue. Because skin is anisotropic and layered (the layers are the epidermis, the dermis, the subcutaneous fat, and the underlying muscle), it is important to understand how jet injections affect each of the layers and how injections should be tailored for specific depths. The scope of this thesis is therefore twofold: the first part seeks to characterize the injection process to understand how the different injection parameters affect injectate distribution and tissue damage in layered skin. The second part of this thesis focuses on the development of the jet injector for needle-less interstitial fluid acquisition. 


\subsection{Chapter Descriptions}

Chapter 2 describes the development of the jet injector device for interstitial fluid acquisition. The software is overhauled as a deterministic state machine and safety features are added. The updated system with the new software is characterized.

Chapter 3 is an investigation of the effect of jet injection on tissue. Injection depth experiments on post-mortem porcine skin are performed. A micro-CT imaging study presents the effect of injection parameters on injectate delivery to different skin layers, and a histological study examines the tissue damage caused by jet injection.

Chapter 4 describes the development of a high-speed X-ray imaging system for the real-time visualization of jet injections in tissue. High-speed videos of both needle and jet injections into tissue are recorded, and the dynamics of the injection are quantified.

Chapter 5 presents a finite element model that simulates the effect of different injection and extraction parameters on the ISF extraction process. The model accounts for the change in tissue permeability due to swelling and compression, and bases the model of solute transport on flow through soils.

Chapter 6 presents experimental work evaluating the effect of different injection and extraction parameters on the ISF acquisition process. Experiments are done on post-mortem porcine skin, and results are compared to the modeling results. The lessons learned from both the simulations and the post-mortem experiments are then applied to a different animal model: the rat. A feasibility study demonstrating the ISF acquisition method on live rats is presented.

Chapter 7 summarizes the contributions of this thesis and gives suggestions for possible areas of future work. 


\section{Chapter 2}

\section{Device Development}

Several modifications were made to the jet injector developed in the BioInstrumentation Lab, most notably to the software. The software both controls the position of the Lorentz-force motor and manages the state of the JI. This chapter describes the changes made to the software to enhance reliability and safety, and presents the characterization of the updated system.

\subsection{Device description}

The jet injector device, shown in Figure 2-1, was similar to a previously described BioInstrumentation JI [27], and consisted of a custom-made linear Lorentz-force motor (using an N52 neodymium magnet in a custom-built housing), a linear potentiometer used as a position sensor, and a commercially available drug ampoule (Injex Ampoule, part \#100100). The Lorentz-force motor coil was wound from 28 gauge wire, and had a resistance of $12.25 \Omega$ and an inductance of $8.07 \mathrm{mH}$. The housing was fixed onto an adjustable vertical linear stage. A tissue mounting stage included a load cell to measure the contact force that the JI applied to the tissue. The $B l$ force constant of the motor measured in a vertical configuration was $11.8 \mathrm{~N} / \mathrm{A}$ downward, and $8.2 \mathrm{~N} / \mathrm{A}$ upward. The force constant was lower when the motor was moving upward (against gravity) due to the weight of the coil. 


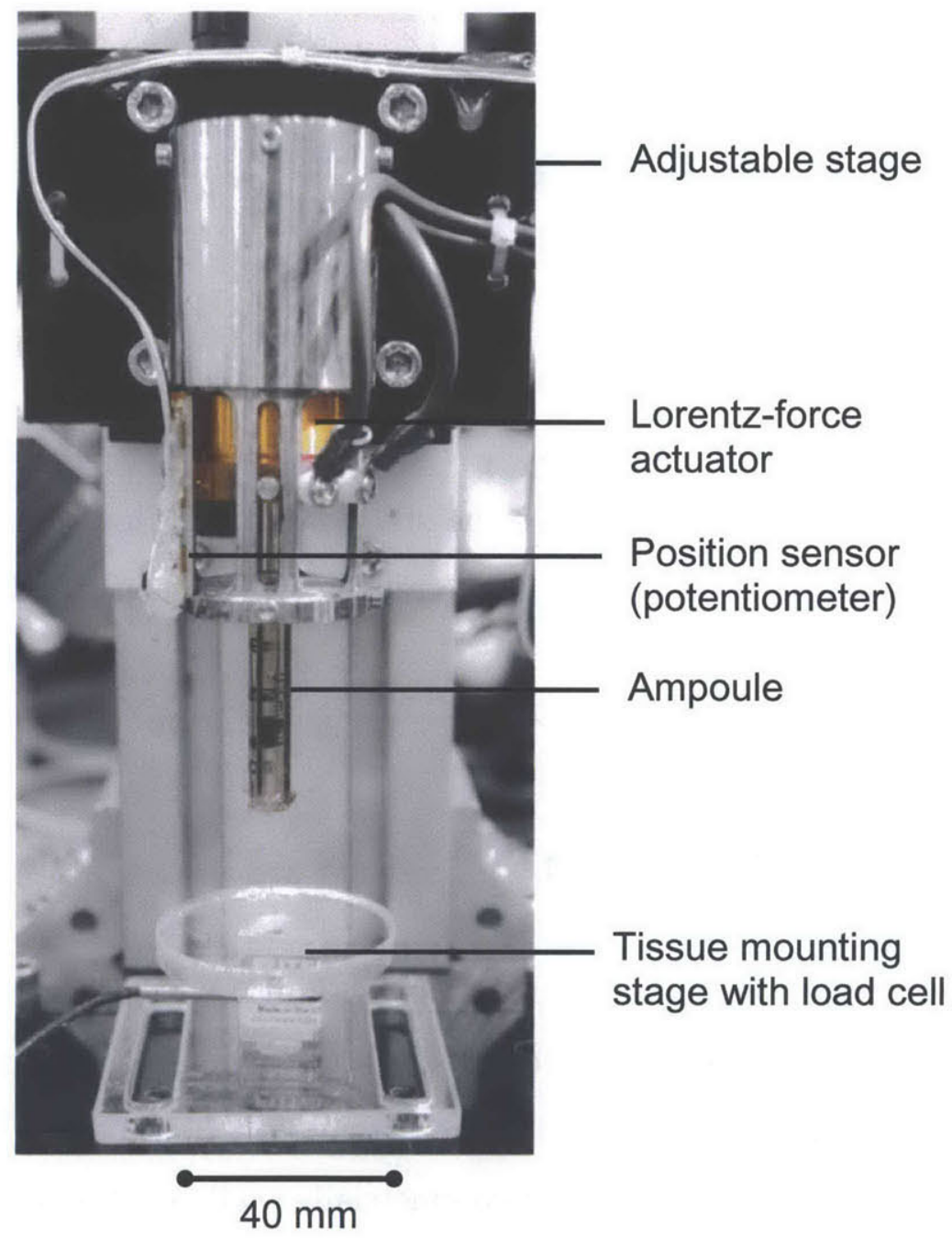

Figure 2-1: Jet injector benchtop device. 


\subsubsection{Control System Hardware}

The control system hardware was updated with a faster controller and support for fixed-point data (the legacy hardware was only able to support 32-bit integers). The updated control system used a compact reconfigurable input-output (cRIO) system comprising a real-time controller (cRIO-9024, National Instruments) embedded in a reconfigurable field-programmable gate-array (FPGA) chassis (cRIO-9113). The modularity and customizability of the cRIO system allowed for quick prototyping of the benchtop apparatus.

Replaceable I/O modules in the cRIO chassis provided analog input and output channels as well as digital input-output channels. An NI 9215 analog input module (with four 16-bit channels), an NI 9263 analog output module (with four 16-bit channels) and an NI 9403 bidirectional digital input/output module (with $32 \mathrm{I} / \mathrm{O}$ channels) were used. Two linear power amplifiers (AE Techron 7224) configured in series (bridge mono mode) were used to amplify the analog control signal and drive the actuator.

\subsubsection{Control System Software}

The legacy software was completely overhauled as a number of improvements were needed to increase reliability and make the software easily modifiable for different applications. The new software was written as a deterministic state machine and was designed to provide a common framework for several different JI applications, as certain functions (e.g. safety features, mode and function selection, data recording) are the same across all applications. The modularity of the new software allowed for easy incorporation of different control schemes, which could be selected depending on the application.

The updated software was written in National Instruments Laboratory Virtual Instrumentation Engineering Workbench (LabVIEW) 2011 and consists of a real-time "host" application which interacts with the FPGA "target" application, interprets

user commands, and provides feedback. The target application consists of an analog 
supervisor which monitors the analog channels, a digital supervisor which monitors the digital channels and arbitrates the state of the system, and a state executor which contains the code to implement each state. A flow chart illustrating the state machine is shown in Figure 2-2.

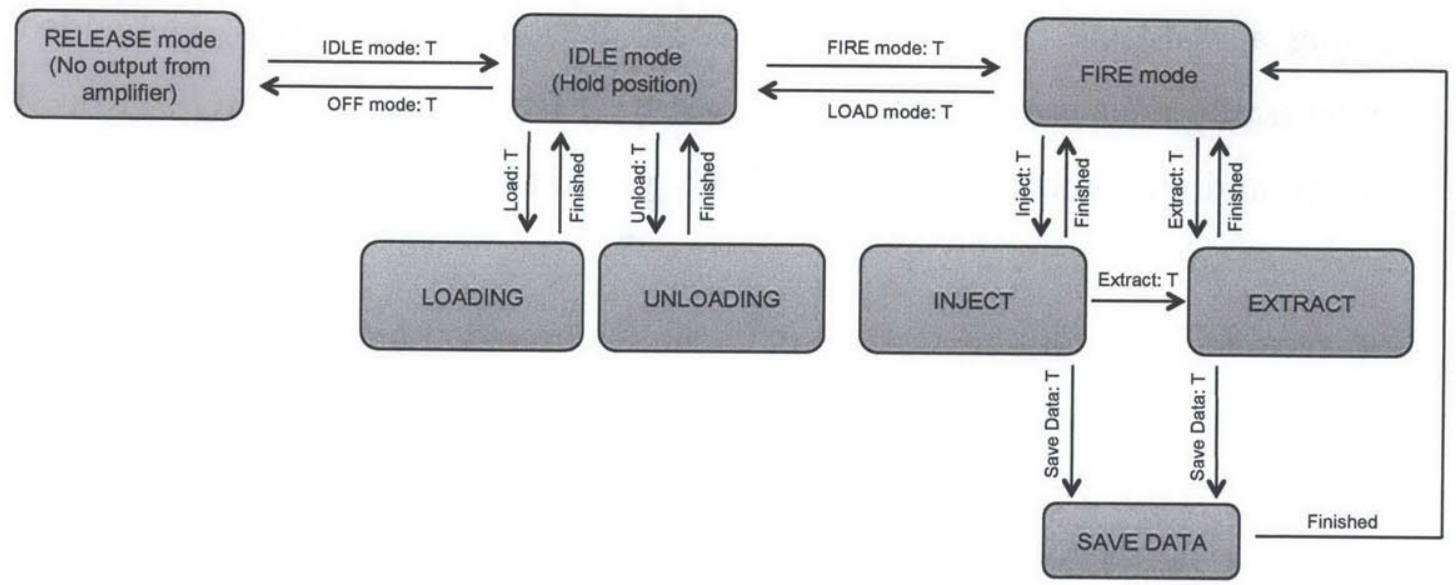

Figure 2-2: Flow chart illustrating the state machine for the new software.

The host application interprets user commands such as which type of controller to use (e.g. feedforward or bang-bang control). It also generates the injection and extraction waveform based on user input. To send a waveform to the FPGA, the host application fills a first in, first out (FIFO) buffer, which is then read by the target application. To save data collected from the position transducer and the amplifier voltage and current monitors, the target application fills a Target-to-Host FIFO while the host simultaneously downloads the data and stores it on the FPGA.

The controller for the loading/unloading of the ampoule and the extraction function was a proportional-integral-derivative (PID) controller. The controller for the injection function was a feedforward controller for improved trajectory following, along with a feedback controller for stability and disturbance rejection. System identification of the motor, piston, and ampoule system was performed to determine the proper gains for the PID controller. A chirp signal containing frequencies ranging from $5 \mathrm{~Hz}$ to $600 \mathrm{~Hz}$ was used as the input and the potentiometer output was measured. The data were used to estimate the parameters of a third order linear model using an iterative prediction-error minimization (PEM) method (Figure 2-3). The data show 
that potentiometer noise dominates the system at frequencies above $1000 \mathrm{rad} / \mathrm{s}$. The system model was calculated to be:

$$
G(s)=\frac{\text { Potentiometer output }(\mathrm{V})}{\text { Amplifier output }(\mathrm{V})}=\frac{2.1964}{s(0.0054 s+1)(0.00123 s+1)} .
$$
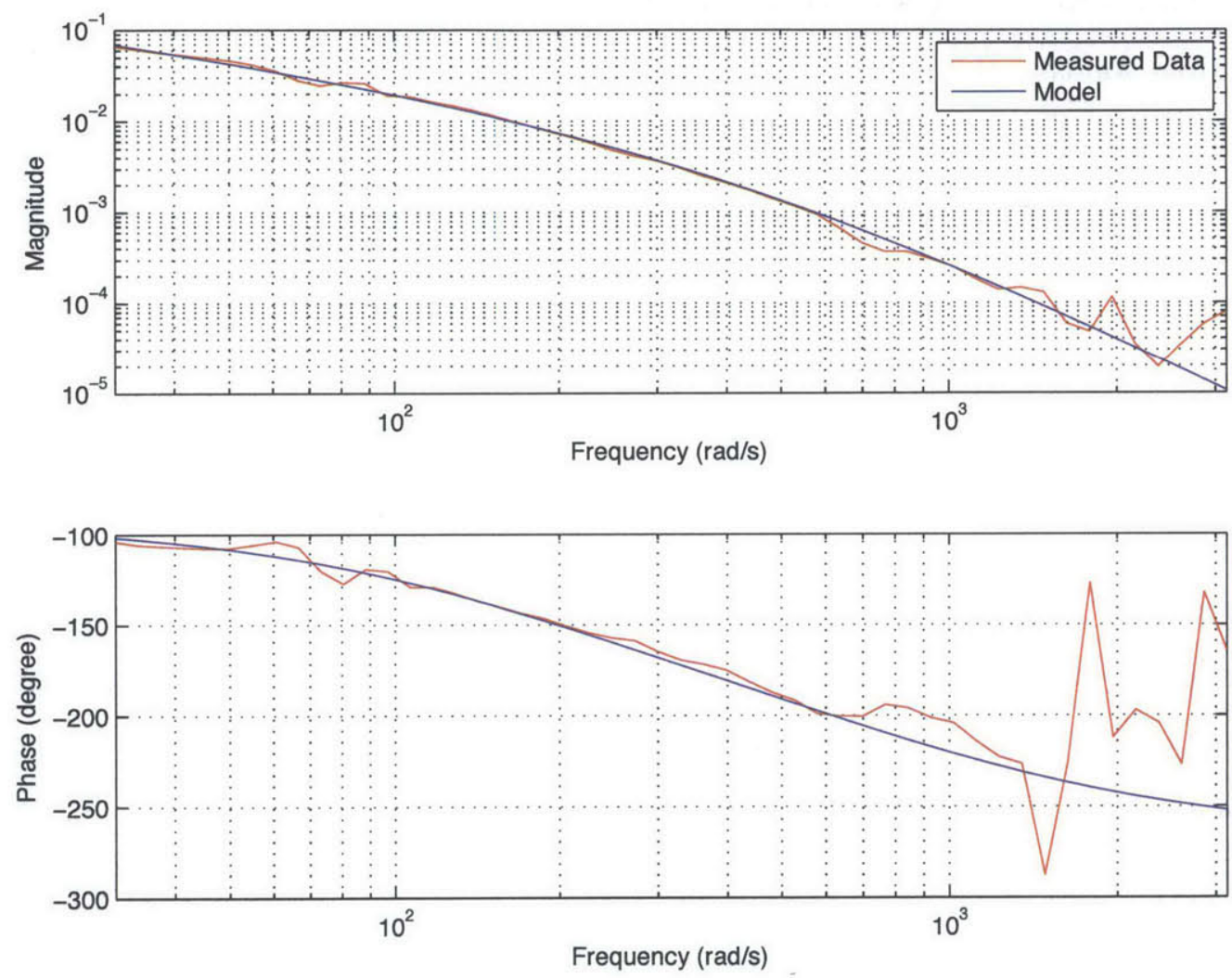

Figure 2-3: Magnitude and phase data used to fit a third order linear model to describe the coil and ampoule dynamics.

The gains for the PID controller were then determined using the system model and loop-shaping methods.

\section{Safety Features}

A major problem with the legacy software was the lack of safety features to prevent coil burnout, as the coil would overheat if the controller went unstable or if the motor stalled. A number of safety features were written into the software to prevent such situations. The amplifier will only output the maximum power during the time course 
of the injection after which the gain is automatically reduced from 40 to 1 , even if the piston has not reached its desired position at the end of the injection. Upper and lower travel limits (defined by the user) were programmed into the software so that it was impossible to build a waveform that caused the piston to travel outside of these limits. The software also automatically cut the signal to the amplifier and shut itself down if the piston travelled outside the user-defined travel limits. Since it is possible for the JI to fire twice for injections that lasted less than $100 \mathrm{~ms}$, if the injection finished before the user removed his/her finger from the trigger button, the software automatically set a $500 \mathrm{~ms}$ wait time between injections to prevent this from occurring.

\section{Injection control software}

The legacy software computed the entire injection waveform on the real-time controller and sent the resultant waveform to the FPGA target. The new software was written such that the real-time controller computed the values of relevant parameters required to generate the piecewise function and sent the parameters to the FPGA. The FPGA then computed one setpoint per timed cycle. In this thesis, the coil position waveform was formed by sending the FPGA the accelerations and timepoints of acceleration, and integrating the acceleration twice (Figure 2-4). The velocity waveform generated was used to calculate the amplifier output using a feedforward model.

The bulk velocity of the jet at the instant it exits the ampoule orifice was calculated from the coil velocity by the continuity equation. It was assumed that the piston tip velocity was equal to the coil velocity (i.e. piston tip compression is negligible), which was determined by differentiating the output of the position sensor. The continuity equation gives:

$$
\rho_{1} A_{1} v_{1}=\rho_{2} A_{2} v_{2}
$$

where $\rho$ is the density of water, $A$ is the cross-sectional area, and $v$ is the fluid velocity. 

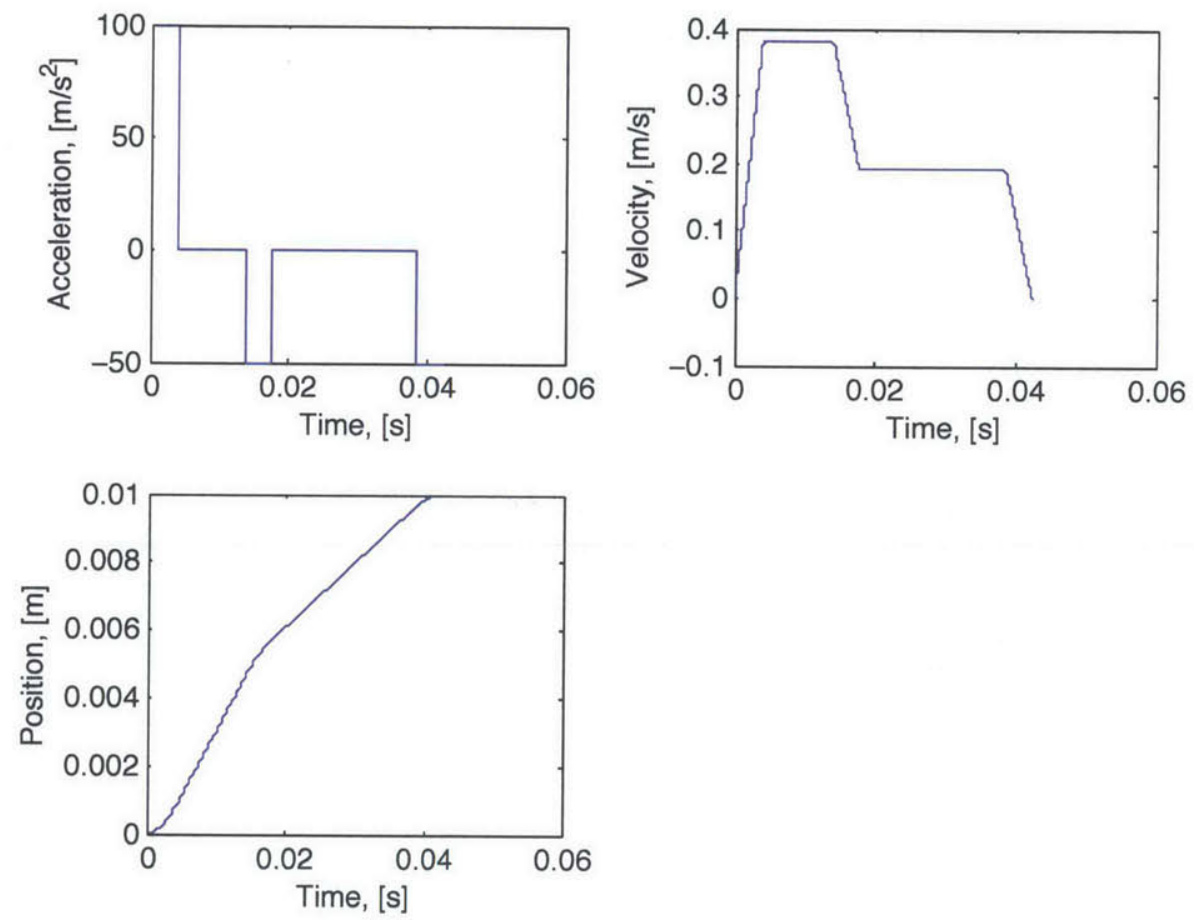

Figure 2-4: The FPGA first builds the acceleration waveform (top left) and then generates the corresponding velocity (top right) and position (bottom left) waveforms by integration. 
This gives:

$$
v_{\text {orifice }}=\left(\frac{D_{\text {piston }}}{D_{\text {orifice }}}\right)^{2} v_{\text {piston }}
$$

where $v_{\text {orifice }}$ is the bulk velocity of the jet at the instant it exits the ampoule orifice, $D_{\text {piston }}$ is the diameter of the piston, $D_{\text {orifice }}$ is the diameter of the orifice, and $v_{\text {piston }}$ is the velocity of the piston.

The feedforward controller was similar to the legacy controller. A voltage to velocity relationship was determined experimentally by a calibration procedure that delivered a set voltage to the motor and measured the resulting steady-state velocity. The results from an example calibration are shown in Figure 2-5. A second order polynomial was fit to the data, and the FPGA used this relationship to calculate the voltage to deliver to the motor based on the desired velocity. A block diagram for the injection controller is shown in Figure 2-6.

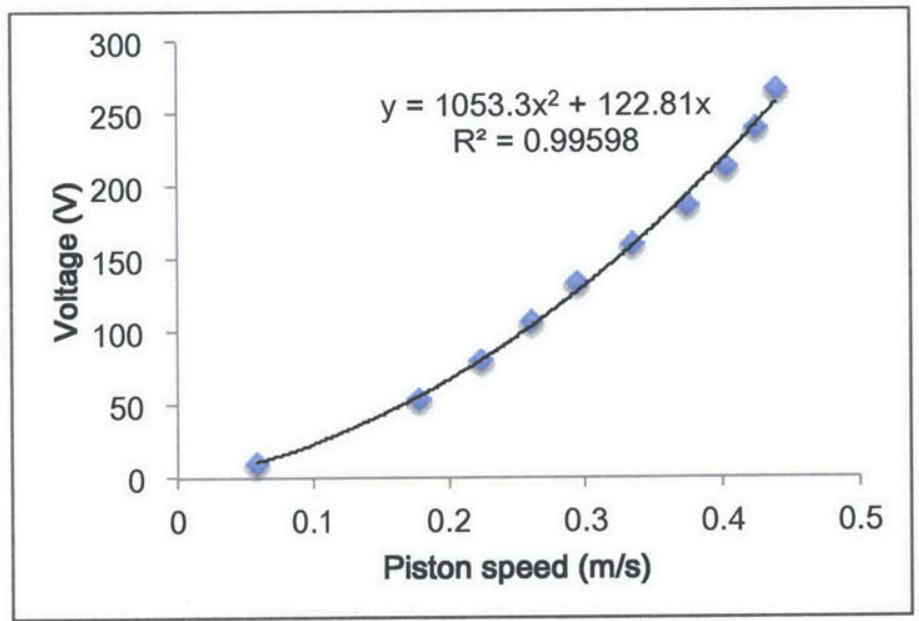

Figure 2-5: Results from a voltage-velocity calibration.

\section{Characterization}

The accuracy of delivering a desired volume of fluid at a set velocity was tested. The volumes of ejected water for set jet velocities ranging from 50 to $150 \mathrm{~m} / \mathrm{s}$ were measured. The average delivered volume errors are shown in Figure 2-7 and the average tracking errors are shown in Figure 2-8. The average volume errors were less than $2 \%$ and the average tracking errors remained less than $3 \%$. 


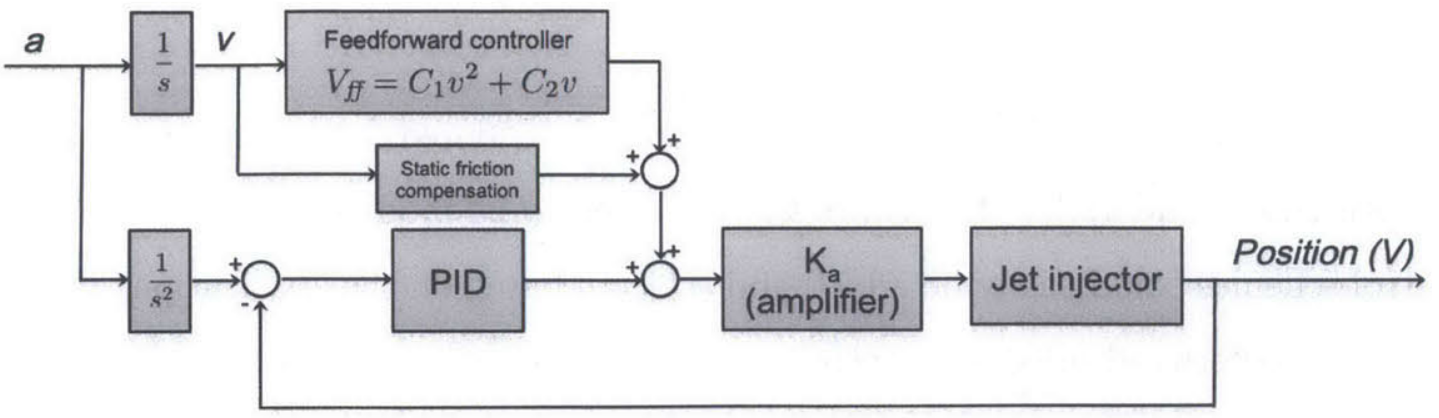

Figure 2-6: The injection controller used a velocity-voltage feedforward controller along with a PID feedback controller.

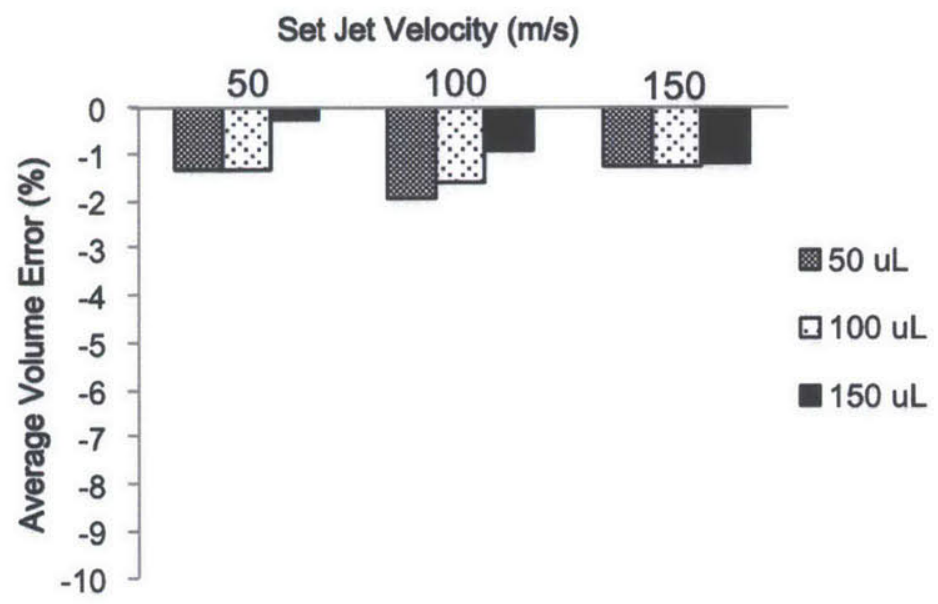

Figure 2-7: Average ejected volume errors.

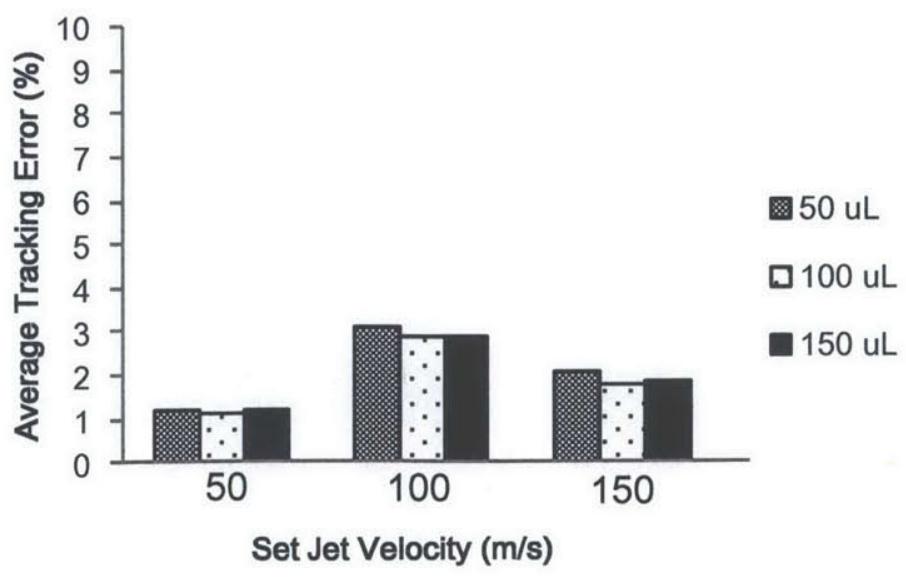

Figure 2-8: Average tracking errors. 


\subsection{Summary}

The jet injector hardware and software were both updated for increased reliability and precision. The updated software was designed to be modular such that different control schemes could easily be added and selected based on the application. For this thesis, a feedforward injection controller was used, resulting in less than $3 \%$ average tracking error. 


\section{Chapter 3}

\section{Injection Studies}

The salient advantage of the Lorentz-force actuated jet injector over other commercially available JIs is that the Lorentz-force actuated JI is servo-controlled. This allows the user to define a custom injection velocity waveform. The unique ability to define a custom waveform allows for the same JI design to be used for a number of different applications. For example, the JI is being investigated for intraocular jet injections, which require a high initial jet velocity to pierce the sclera and a slow following velocity to avoid retinal damage [28]. The injection waveform required to deliver drugs to the eye with minimal damage is markedly different from that of skin injections, so the ability to easily adjust the injection parameters is critical.

Moreover, users can tailor the injection waveform to target specific depths and possibly enhance drug uptake by controlling the dispersion of the injectate in the tissue. The device described in this thesis uses a two-phase injection waveform shown in Figure 3-1. The first phase defines the initial jet velocity $\left(v_{j e t}\right)$ and time at this velocity $\left(t_{j e t}\right)$ required to puncture the tissue. The second phase defines a slower follow-through velocity $\left(v_{\text {follow }}\right)$ that defines the total delivered fluid volume ( Vol) and allows for delivery at a lower pressure to reduce tissue damage and associated pain. It is widely believed that the first high velocity phase is largely responsible for the injection depth, while the slower follow-through phase affects injection dispersion. It should be noted that the new software described in Chapter 2 defines $t_{j e t}$ slightly differently than the legacy software (used in [27]). The legacy software defined $t_{j e t}$ as 
the time of the high-velocity phase, and included the time spent accelerating to $v_{j e t}$ and decelerating to $v_{\text {follow. }}$. The new software defines $t_{j e t}$ as the amount of time spent at peak velocity $v_{j e t}$ (does not include acceleration and deceleration time).

The use of a two-phase waveform was first reported in 2006 when the waveform was tested using a previous pressure-controlled version of the JI device [29, 30]. Earlier work done by others had suggested that the jet injection process occurs in distinct phases: an initial puncture phase where an erosion hole is formed, and a filling phase where the injectate is dispersed into the medium [31]. Studies done with the pressurecontrolled JI device found that injection depth was highly correlated to peak pressure in polyacrylamide gels and post-mortem tissue: higher peak pressure resulted in deeper injection depths $[29,30,32]$.

The JI device described in this thesis calculates the injection waveform by the user-defined parameters $v_{j e t}, t_{j e t}, v_{\text {follow }}$, and Vol. The user can also define the piston acceleration (acc) and deceleration (dec). As $v_{j e t}$ and $v_{\text {follow }}$ are analogous to peak pressure and follow-through pressure, injection depth is highly dependent on the magnitude of $v_{j e t}[27]$. High-speed video imaging of jet injections into clear polyacrylamide gels confirmed the roles of $v_{j e t}$ and $v_{\text {follow }}$ [32]. Stachowiak et al. and Taberner et al. also reported the dependence of injection depth on $t_{j e t}$ : longer $t_{j e t}$ will result in deeper injection depths $[33,27]$.

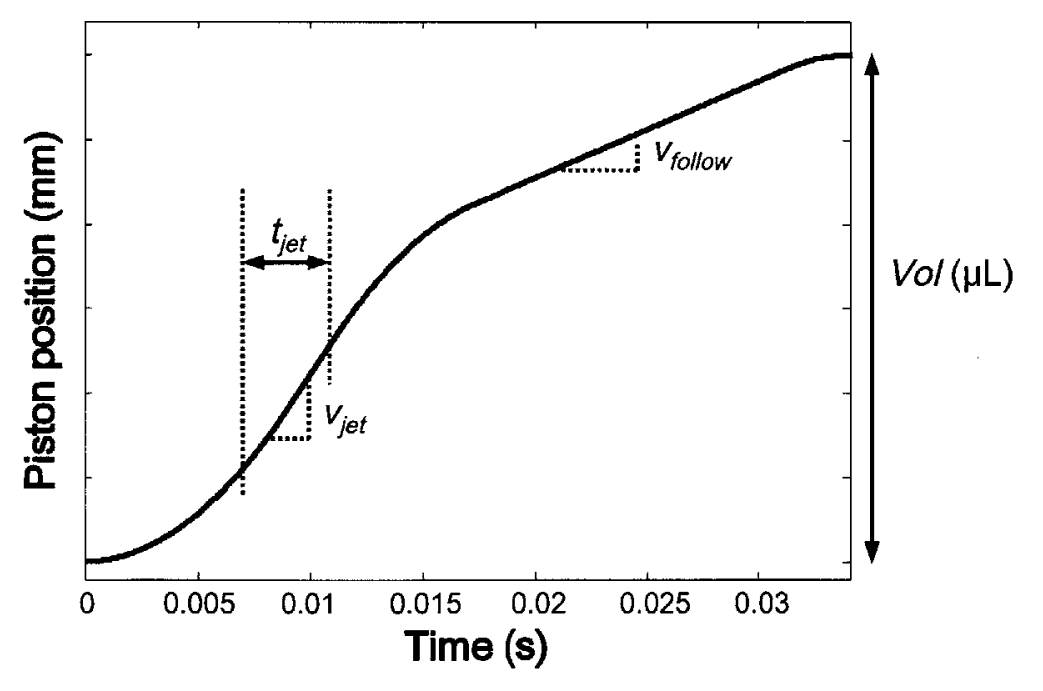

Figure 3-1: Two-phase waveform used for jet injections. 
The majority of jet injection studies have to this date been on clear gel analogs, and most studies so far have focused on the effects of $v_{j e t}$ and $t_{j e t}$ on injection depth. There is limited data on how factors such as contact force will affect delivery. Additionally, due to the inhomogeneous layered structure of skin it is unclear how injection parameters such as $\mathrm{Vol}$ and $v_{\text {follow }}$ will affect delivery. It is also uncertain how the different anisotropic material properties of each skin layer will influence the formation of the penetration hole. The injection process into tissue must be characterized before the jet injector can be developed for ISF acquisition.

\subsection{Background: Skin Layers}

The skin consists of several layers: the outermost layer being the epidermis, followed by the dermis, the subcutaneous fat, and the muscle (Figure 3-2). In this thesis, the subcutaneous fat and the muscle layer will be included in the general description of "skin."

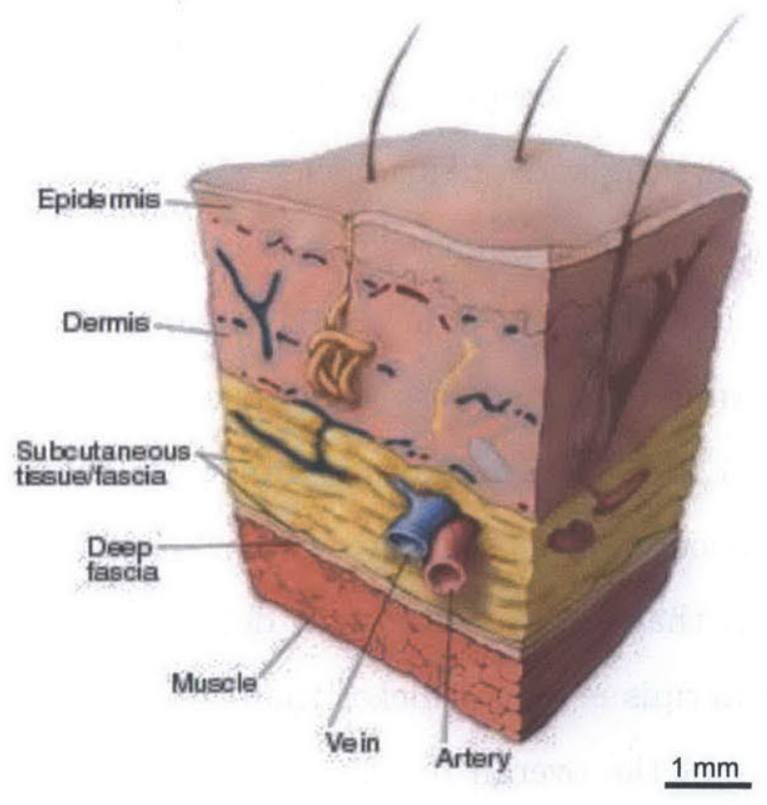

Figure 3-2: The structure of skin is layered and consists of the epidermis, the dermis, the subcutaneous fat, and the muscle. Figure modified from [2]. 


\subsubsection{Microstructure}

Because a controllable jet injector has the potential to target specific skin layers, jet injection waveforms can be tailored for specific drugs, as different drugs are generally delivered by needle to specific sites. Additionally, the jet injection waveform can be optimized for dispersion patterns that enhance drug uptake. To understand jet injection dispersion patterns, it is important to understand the microstructure of the skin.

The epidermis is $50-100 \mu \mathrm{m}$ thick, and contains Langerhans cells, which process antigen fragments and produce an immune response. The primary function of the epidermis is to protect the body from infection and dehydration.

The dermis is typically 1-4 mm thick and consists of a dense network of collagen fibers as well as elastin, glycosaminoglycans (GAGs, which are long unbranched polysaccharides), and proteoglycan core proteins (PGPs, which are GAGs covalently bonded to proteins). A major component of the GAGs contained in the dermis is hyaluronate, a hydrophilic molecule that binds most of the tissue fluid, thus rendering the dermis as a gel-like material. There is a small amount of free movable fluid (i.e. interstitial fluid) that is not bound in the gel or cells. The dermis may also contain blood vessels, sweat glands, hair follicles, and mechanoreceptors. The dermis is divided into two layers: the papillary layer that lies directly beneath the epidermis, and the deeper reticular layer (Figure 3-3). The boundary between the papillary dermis and the epidermis is shaped into numerous papillae, which contain densely interwoven, fine $(0.3-3 \mu \mathrm{m}$ thick) bundles of randomly oriented collagen fibers [3]. The reticular dermis consists of loosely interwoven, thick collagen bundles, typically $10-40 \mu \mathrm{m}$ in diameter, that form a strong but deformable three-dimensional lattice $[3,34]$. The reticular dermis is much thicker than the papillary dermis; the reticular dermis comprises $90 \%$ of the overall dermis thickness. The boundary between the two layers is indistinct.

The collagenous structure of the dermis causes its mechanical behavior to be

nonlinear. Specifically, the stress-strain curve of skin is known to be ' $J$ '-shaped (Figure 


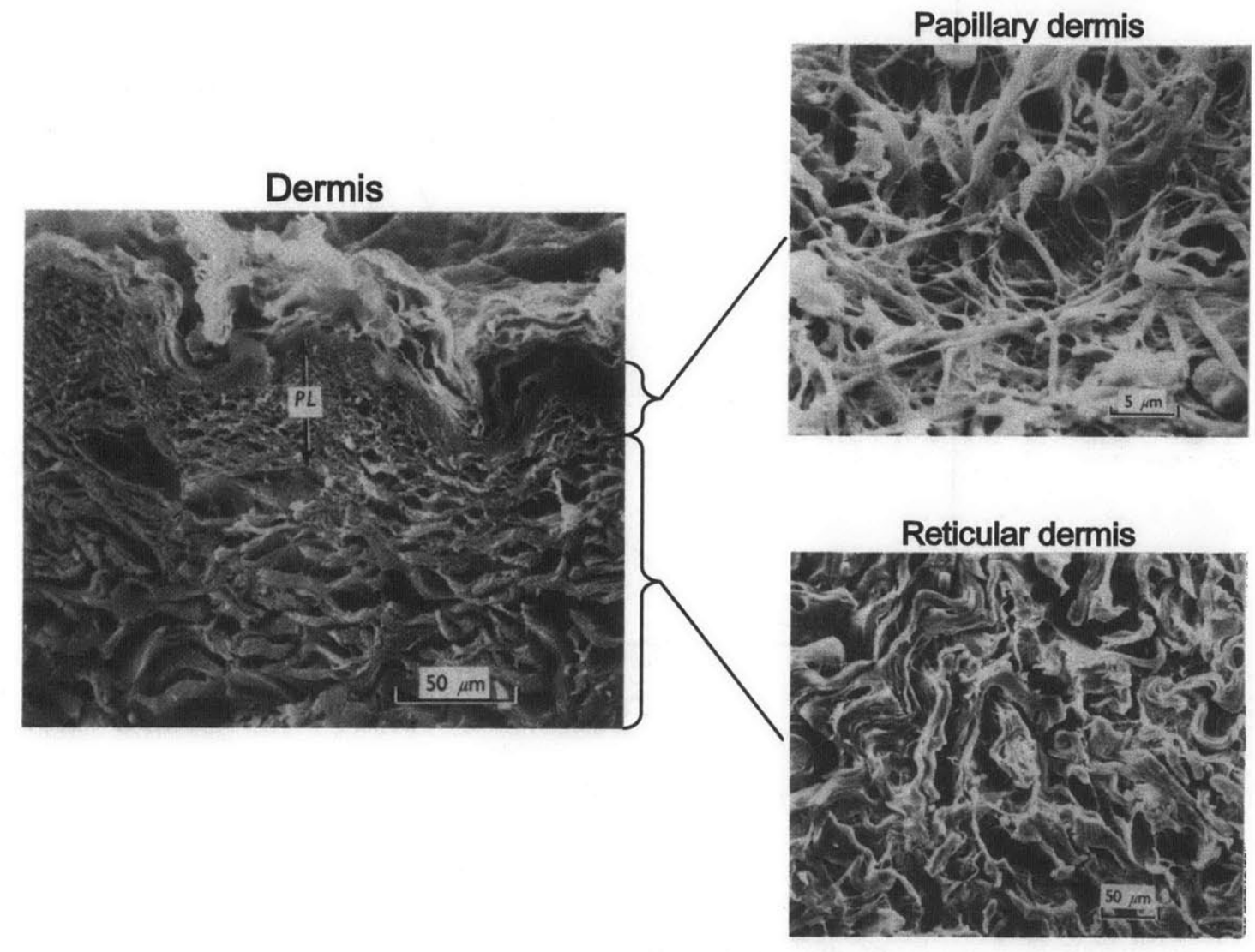

Figure 3-3: Scanning electron microscopy images of the fibrous tissue in the dermis. The denser papillary layer makes up approximately $10 \%$ of the dermal thickness, while the coarser reticular layer makes up the remaining $90 \%$ of the dermis. Figure adapted from [3]. 
$3-4)$. At low strains $(\epsilon<0.3)$, the deformation is due to the elastin in the skin. At this point, the collagen fibrils are beginning to straighten out. For strains between 0.3 and 0.6 , the elastic modulus is much higher as the stiff collagen fibrils start to carry load. At high strains above 0.6, the fibrils have completely straightened out and are carrying all the load.

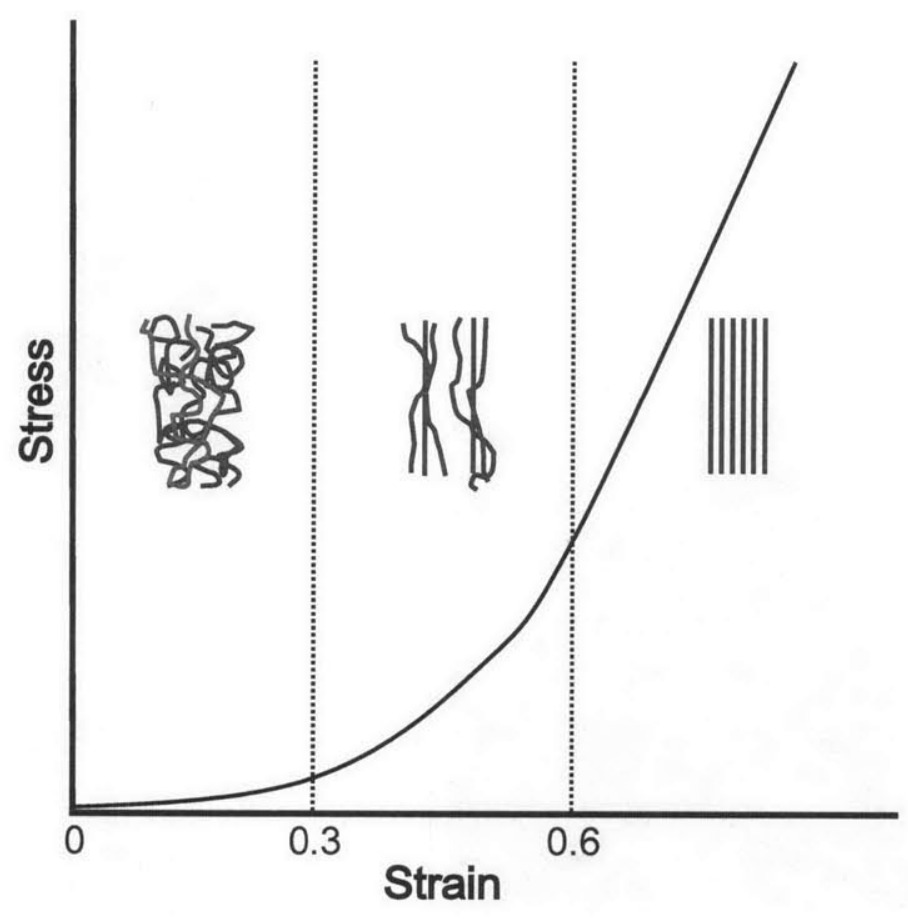

Figure 3-4: The stress-strain curve of a collagen matrix is ' $\mathrm{J}$ '-shaped, with three different zones. Figure from [4].

The subcutaneous layer consists mainly of subcutaneous adipose tissue but also contains blood vessels, lymphatics, and nerves. Subcutaneous tissue is composed of lipid-filled cells called adipocytes organized in an extracellular matrix that has two distinct collagen-based structures (Figure 3-5). The first is the reinforced basement membrane, which consists of $100 \mathrm{~nm}$ thick collagen fibers that surround each adipocyte and resemble a closed-cell foam. The second is the interlobular septa, which act as reinforcement fibers (approximately $2 \mu \mathrm{m}$ thick, spaced $1 \mathrm{~mm}$ apart) of the reinforced basement membrane and resembles an open-cell foam. The reinforced basement membrane is composed of various types of fibrous collagen, while the interlobular septa is composed mainly of Type I collagen. 

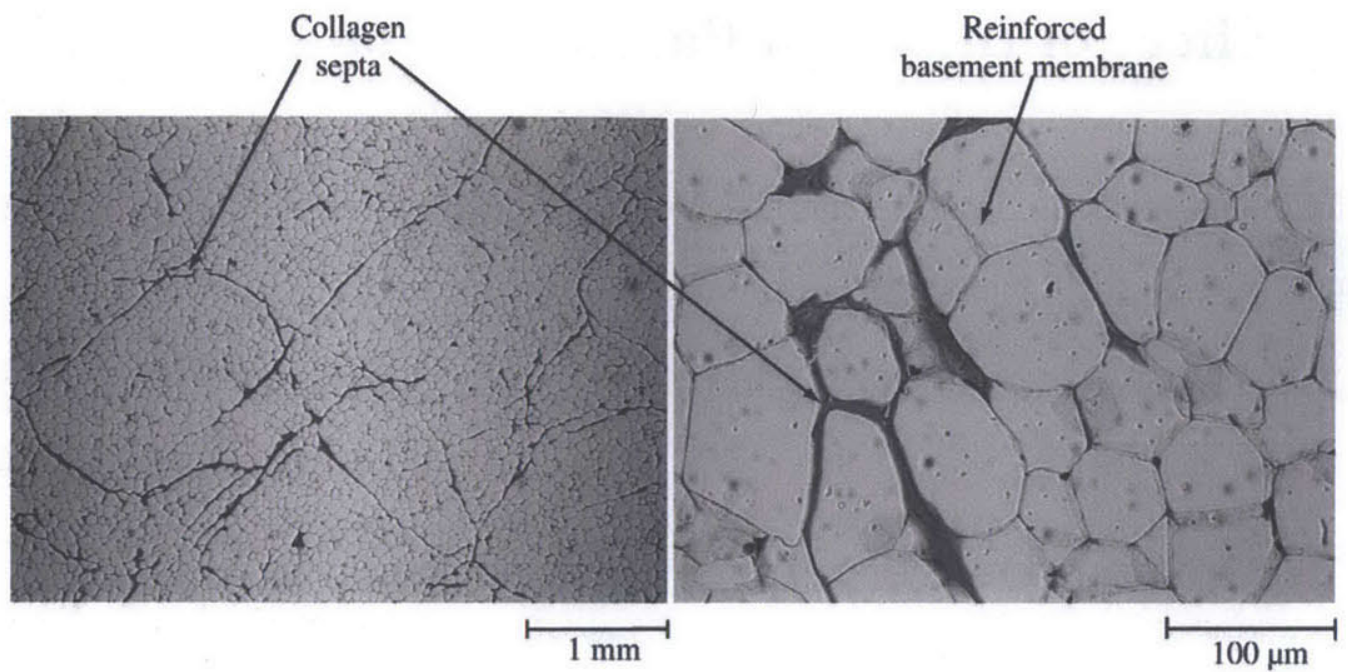

Figure 3-5: Histological section of porcine adipose tissue at two different magnifications. Left image shows septa and right image shows the reinforced basement membrane that surrounds each adipocyte. Figure from [5].

The muscle lies underneath the subcutaneous tissue. Connective tissue organizes the muscle in a hierarchal fashion (Figure 3-6). At the lowest level, muscle fibers are organized by a network of connective tissue called the endomysium, analogous to the connective tissue that surrounds adipocytes in the subcutaneous tissue. A sheath of connective tissue called the perimysium surrounds muscle fibers (and the endomysium), organizing them into bundles, or fascicles. The epimysium is connective tissue that surrounds the entire muscle. Nerves travel through the epimysial and perimysial septa.

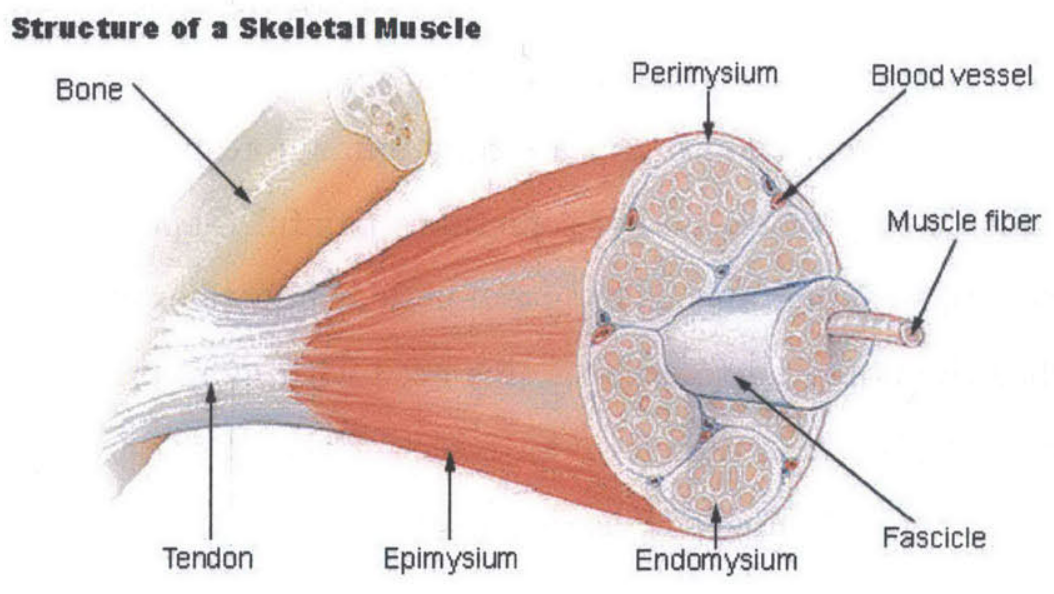

Figure 3-6: The structure of skeletal muscle. 


\subsection{Effect of Injection Parameters on Injection Depth}

The following sections describe the effect of nozzle contact force and total injection volume ( $\mathrm{Vol}$ ) on dispersion depth.

\subsubsection{Optimal Delivery Target: the Dermis}

For the application of using the needle-free jet injector for interstitial fluid acquisition, the target injection site is the dermis, as studies have indicated that the level of analytes in dermal interstitial fluid are closely correlated to their corresponding levels in blood. For the wider application of needle-free jet injection of drugs, studies have shown that the dermis is also the optimal delivery site for vaccines such as influenza and Hepatitis B. Holland et al. found that intradermal administration of the influenza vaccine resulted in higher seroprotection and seroconversion rates than with conventional intramuscular administration [35]. Henderson et al. found that administering only $1 / 6$ of the standard dose of Hepatitis B vaccine intradermally resulted in similar seroconversion and seropositivity rates when compared to administering the full dose intramuscularly [36].

Immune responses are strong in the dermis due to the presence of a dense network of dendritic cells and an extensive lymphatic system. These both allow for the efficient migration of antigen, resulting in a strong immune response. However, dermal injection is difficult to perform and is sometimes painful (depending on the skill of the administrator), so subcutaneous or intramuscular injections are usually preferred [37]. The JI has the potential to address this need with more precise and controlled delivery to the dermis. It is therefore desirable for the JI to target the dermis for many JI applications, including vaccine delivery and interstitial fluid acquisition. The depth of injection delivery has been shown to be dependent on the values of $v_{j e t}$ and $t_{j e t}$, but the dependence on $\mathrm{Vol}$ also needs to be investigated. 


\subsubsection{Importance of contact force}

A limitation of previous studies on the relationship between injection parameters and injection depth is the lack of data on the role of contact force. Intuition suggests that contact force or pre-stretching of the skin will have a noticeable effect on the resultant injection, but to the best of our knowledge the contact force has not been recorded in previous jet injection studies. Taberner et al. performed their injection depth experiments "by placing the tip of the ampoule in contact with the gel or tissue until completion of the injection" [27]. On the other hand, Stachowiak et al. maintained a separation distance of $1 \mathrm{~mm}$ between the injector nozzle and polyacrylamide gel tissue analogs $[38,33]$. Schramm-Baxter et al. also maintained a separation distance of 1 $\mathrm{mm}$ for their early experiments [31,39], and later varied the separation distance between 1 and $5.8 \mathrm{~mm}[40]$. However, they reported significant backflow when injecting into tissue [40].

While there have been studies that have recorded the level of pre-stretch for needle injections and knife insertions into skin, the results are conflicting. Shergold and Fleck suggested that the conflicting results may be due to the differences in constraint on the skin [41]. They postulated that when a needle is pressed into loose skin, the skin is compressed against the underlying muscle and the penetration force increases, as reported by Ankerson et al. [42]. On the other hand, if the skin is pre-stretched the skin is unsupported by the underlying muscle and the penetration force is low, as reported by Figge and Barnet [43].

Past experience have shown that a higher $v_{j e t}$ is required to penetrate skin when there is a gap between the JI nozzle and the skin. Additionally, for ISF acquisition, the JI must be in contact with the skin to create a seal between the ampoule and the injection site such that a vacuum can be created in the ampoule. Thus, the effect of contact force on dispersion depth was investigated and quantified in the following section. 


\subsubsection{Experimental Methods}

\section{Tissue Preparation}

Post-mortem porcine tissue was obtained through the MIT Tissue Harvest Program using procedures approved by the MIT Committee on Animal Care and in accordance with the NIH Guide for the Use and Care of Laboratory Animals. Tissue was harvested from the abdomen of Yorkshire pigs approximately six months in age immediately after euthanasia and included muscle, subcutaneous fat, dermis, and epidermis. The samples were vacuum-sealed and stored at $-80^{\circ} \mathrm{C}$. Prior to experimentation, the samples were thawed to room temperature, and the skin surfaces were carefully cleaned with water.

\section{Dispersion depth measurements}

The tissue was placed onto a tissue mount stage that contained a load cell. The JI was then lowered such that the tip of the ampoule was in contact with the surface of the skin, and the contact force was measured. An injection was performed, and the tissue was medially sectioned and the dispersion depth was measured. The dispersion depth was defined as the maximum depth the injectate had spread into the tissue. Data sets were compared using the two tail $t$-test for unequal variances.

\subsubsection{Results and Conclusions}

The effect of contact force on dispersion depth for various values of $v_{j e t}$ is shown in Figure 3-7. The dispersion depths for contact forces of $200 \mathrm{mN}$ and $800 \mathrm{mN}$, and $v_{j e t}$ of $150 \mathrm{~m} / \mathrm{s}$ and $175 \mathrm{~m} / \mathrm{s}$ were measured. The dispersion depth for $v_{j e t}=200 \mathrm{~m} / \mathrm{s}$ with $800 \mathrm{mN}$ contact force was not measured, since these parameters resulted in dispersion depths that were larger than the tissue sample thicknesses. It is clear that a contact force of $800 \mathrm{mN}$ resulted in deeper dispersion than a contact force of $200 \mathrm{mN}$. Additionally, as expected, higher values of $v_{j e t}$ resulted in deeper dispersion depths. Qualitatively, the tip of the ampoule was in gentle contact with the skin surface for a contact force of $200 \mathrm{mN}$, while the ampoule was firmly pressed into the 
skin with a contact force of $800 \mathrm{mN}$.

For interstitial fluid acquisition, it is important to maintain an airtight seal between the skin surface and the ampoule. Initial, quick feasibility experiments showed that a better seal was maintained with the larger contact force of $800 \mathrm{mN}$. Thus, for all subsequent post-mortem tissue experiments, a contact force of $800 \mathrm{mN}$ was used.

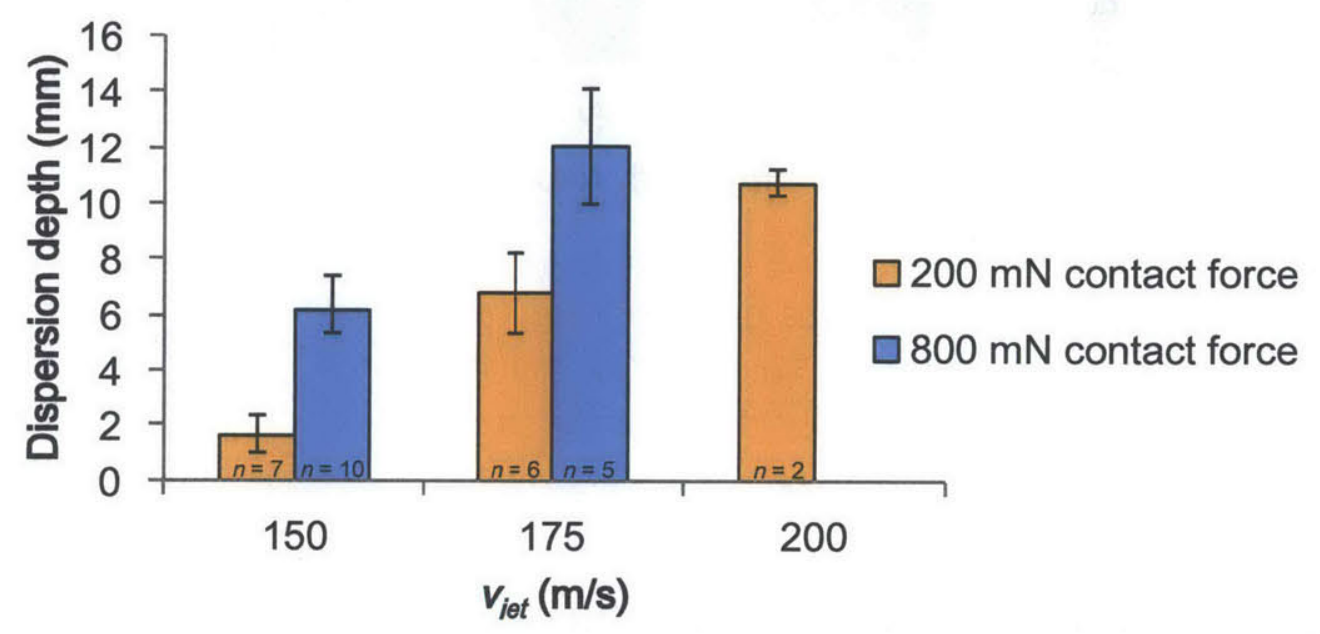

Figure 3-7: The dispersion depths measured for various values of $v_{j e t}$ and contact force. For this set of experiments, $v_{\text {follow }}=50 \mathrm{~m} / \mathrm{s}$ and $\mathrm{Vol}=100 \mu \mathrm{L}$. The error bars represent standard errors.

The dependence of dispersion depth on $\mathrm{Vol}$ for $v_{\text {jet }}=150 \mathrm{~m} / \mathrm{s}$ is shown in Figure 3-8. Delivery volumes of $30 \mu \mathrm{L}, 50 \mu \mathrm{L}$, and $100 \mu \mathrm{L}$ were tested with $v_{j e t}=150 \mathrm{~m} / \mathrm{s}$, $t_{\text {jet }}=2 \mathrm{~ms}$, and $v_{\text {follow }}=50 \mathrm{~m} / \mathrm{s}$. A delivery volume of $30 \mu \mathrm{L}$ resulted in inconsistent penetration. The difference in dispersion depth for $V o l=50 \mu \mathrm{L}$ versus $\mathrm{Vol}=$ $100 \mu \mathrm{L}$ was not statistically significant. This can be attributed to the injectate following the path of least resistance, spreading along the plane between the subcutaneous fat layer and the muscle layer rather than spreading deeper into the muscle. For these particular tissue samples, the boundary between the subcutaneous fat and the muscle layers was located at a depth of approximately $6 \mathrm{~mm}$.

Similarly, the dispersion depth dependence on $V o l$ for $v_{j e t}=175 \mathrm{~m} / \mathrm{s}$ is shown in Figure 3-9. The results in Figure 3-9 show that a higher injection volume of 100 $\mu \mathrm{L}$ results in deeper dispersion $(p=0.05)$. Once the injectate has reached the muscle layer, a higher injected volume will result in deeper dispersion since there is more 


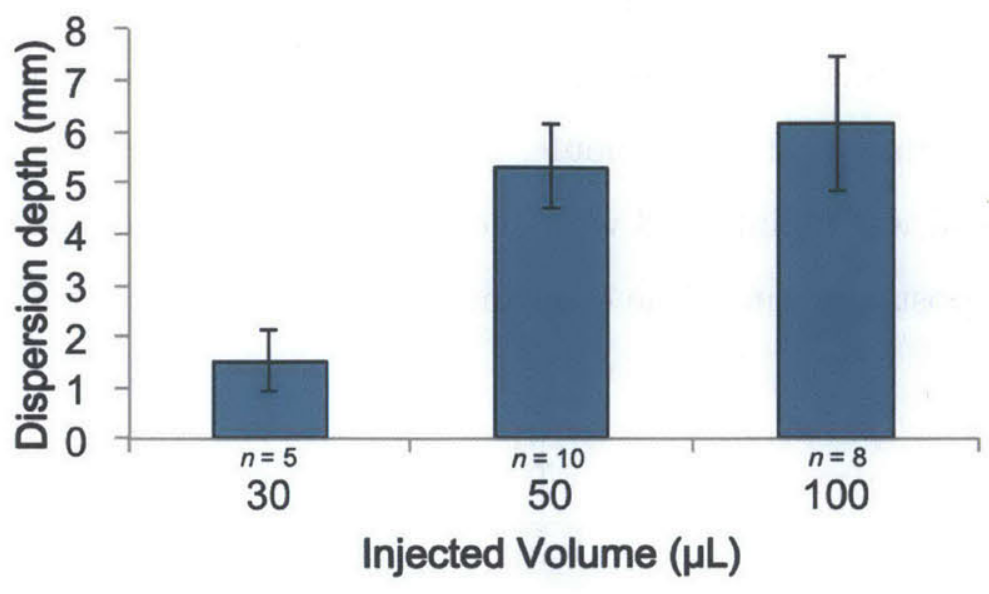

Figure 3-8: The dispersion depths measured for various values of Vol. For this set of experiments, $v_{\text {jet }}=150 \mathrm{~m} / \mathrm{s}, t_{\text {jet }}=2 \mathrm{~ms}$, and $v_{\text {follow }}=50 \mathrm{~m} / \mathrm{s}$. The error bars represent standard errors.

volume to spread out from the penetration hole.

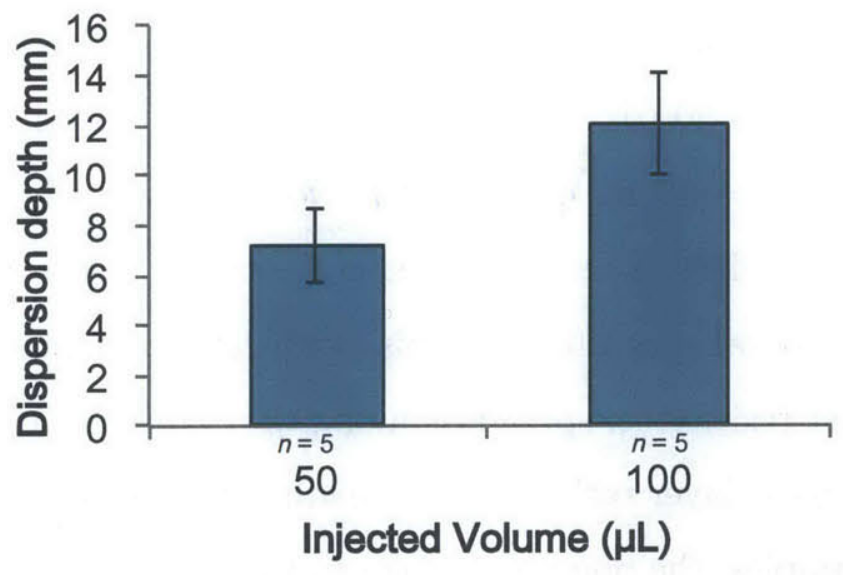

Figure 3-9: The dispersion depths measured for various values of Vol. For this set of experiments, $v_{\text {jet }}=175 \mathrm{~m} / \mathrm{s}, t_{\text {jet }}=2 \mathrm{~ms}$, and $v_{\text {follow }}=50 \mathrm{~m} / \mathrm{s}$. The error bars represent standard errors. 


\subsection{X-ray Microtomography Studies of Jet Injec- tions}

Along with dispersion depth measurements, it is important to know the post-injection dispersion pattern in tissue. The dispersion pattern informs us on where the injectate has traveled and how much injectate has been delivered to certain skin layers. Jet injection dispersion patterns in tissue have typically been examined by injecting dye and either medially sectioning the tissue or by using a camera to image 15-30 $\mu \mathrm{m}$ sections cut on a cryotome. The latter method can be used to reconstruct threedimensional volumes using image recognition software. However, both methods are destructive and depending on the dye used, can be inaccurate due to dye smearing during the cutting process.

$\mathrm{X}$-ray microtomography (commonly known as micro-CT imaging) is a useful tool for nondestructive imaging of biological and nonbiological specimens. A micro-CT scanner consists of a stationary X-ray source and detector, and a stage that rotates the specimen. The images obtained by a micro-CT scanner are typically reconstructed into two-dimensional cross-sectional slices, which can then be stacked to create threedimensional volumes. An advantage of using micro-CT imaging to visualize dispersion patterns in tissue is that the intensity value of each voxel is directly correlated to the density of the material at that location. This information can then be used to deter-

mine the extent of dilution of the injectate in the tissue. Additionally, the intensity value can be used to distinguish between the different layers of the skin without staining. The dermis appears as the densest layer while the subcutaneous fat appears as the least dense layer. This section explores the dispersion patterns resulting from needle and jet injections, and investigates the effect of $v_{\text {follow }}$ on injectate delivery.

\subsubsection{Experimental Methods}

Post-mortem porcine tissue was obtained and prepared by the methods described in Section 3.2.3. For each injection, the JI applied a contact force of $0.8 \mathrm{~N}$ onto 
the skin. The JI injected the tissue with a preset waveform with AuroVist ${ }^{\top M} \mathrm{X}$-ray contrast agent (Nanoprobes, \#1115A). AuroVist ${ }^{\top M}$ was selected as the radiopaque dye since it had a similar viscosity to water. Injected tissue samples were scanned using a Skyscan-1172 micro-CT scanner. Cross-sectional images were reconstructed using NRecon software package. Three-dimensional volumes were reconstructed using ImageJ software.

\subsubsection{Results}

\section{Dispersion in subcutaneous fat}

Porcine tissue samples were first injected with a 27 gauge (410 $\mu \mathrm{m}$ nominal OD) needle to compare dispersion patterns with injections performed with the jet injector. Injections of various volumes (e.g. $35 \mu \mathrm{L}, 80 \mu \mathrm{L}$, and $100 \mu \mathrm{L}$ ) into the subcutaneous fat all resulted in the formation of a spherical bolus. However, the dispersion pattern was non-uniform indicating the fluid does not disperse symmetrically from a point source. Instead, the sponge-like dispersion pattern (Figure 3-10a) indicates that the injectate follows weak paths in the fat during injection. Since fat is organized as groups of weak cells surrounded by stronger sheaths of collagen (described in Section 3.1.1), the dispersion pattern indicates that the injectate will preferentially flow through the weak cells. The injectate will then diffuse into the adjacent tissue. The fluid flow path suggested by the dispersion pattern is shown in Figure 3-10b.

Jet injection dispersion differs from needle injection patterns in that the injectate must travel through the superficial layers of skin before it reaches the desired target. For example, for a subcutaneous fat delivery, the injectate will travel through the dermis, resulting in some dispersion across the dermis. Despite this difference, the dispersion pattern in the subcutaneous fat layer had a sponge-like shape similar to needle injections (Figure 3-11). Changing the value of $v_{\text {follow }}$ did not change the appearance of the dispersion pattern; $v_{\text {follow }}=25 \mathrm{~m} / \mathrm{s}$ and $v_{\text {follow }}=65 \mathrm{~m} / \mathrm{s}$ exhibited no apparent difference in dispersion patterns in the fat layer.

It should be noted that while the bulk of the injectate in the subcutaneous fat 
Side view

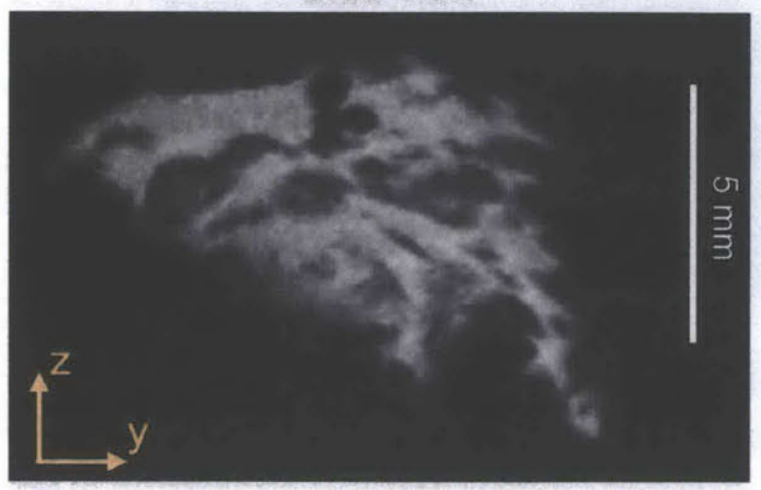

\section{Top view}

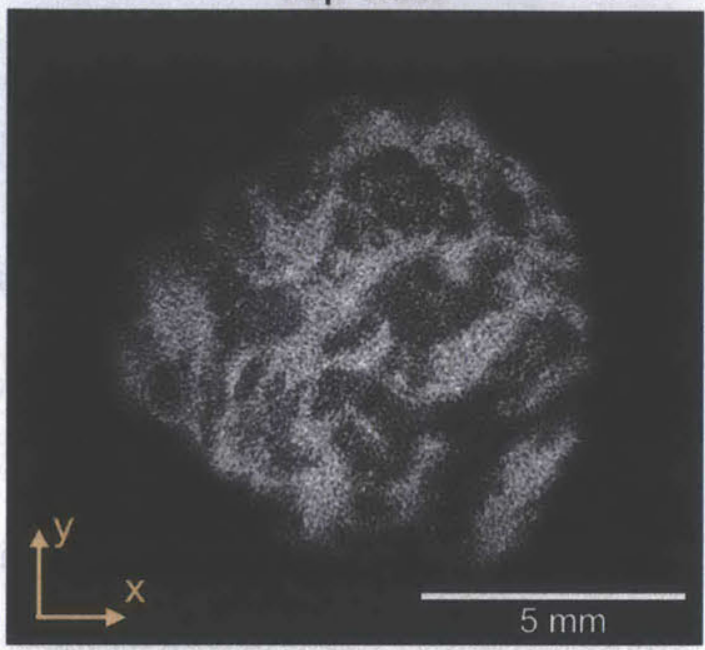

(a) Non-uniform sponge-like dispersion pattern in subcutaneous fat, indicating that the injectate follows weak paths during injection.

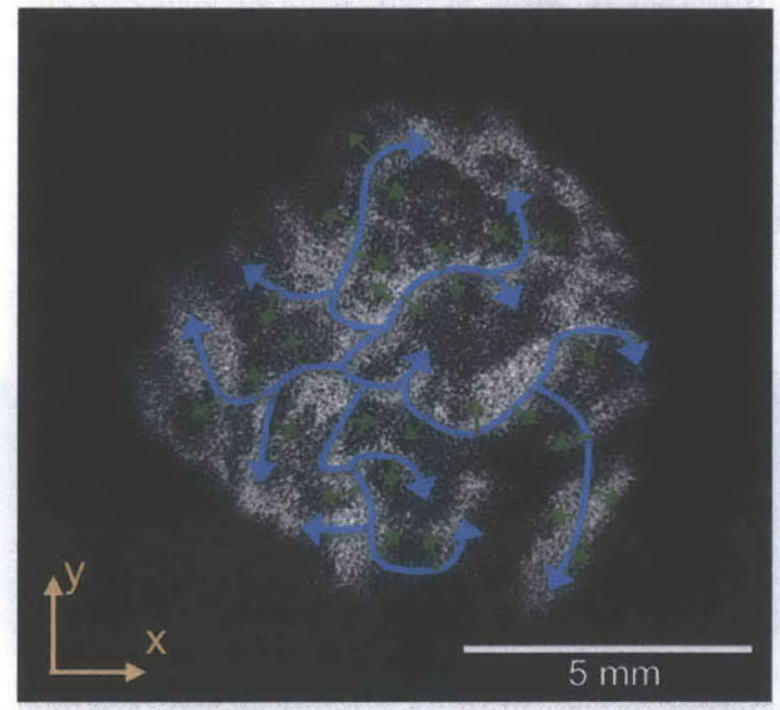

(b) Fluid flow path in subcutaneous fat suggested by the dispersion pattern.

Figure 3-10: Micro-CT images (two-dimensional) of the dispersion pattern for a $100 \mu \mathrm{L}$ injection into the subcutaneous fat layer using a 27 gauge $(410 \mu \mathrm{m}$ nominal OD) needle. 

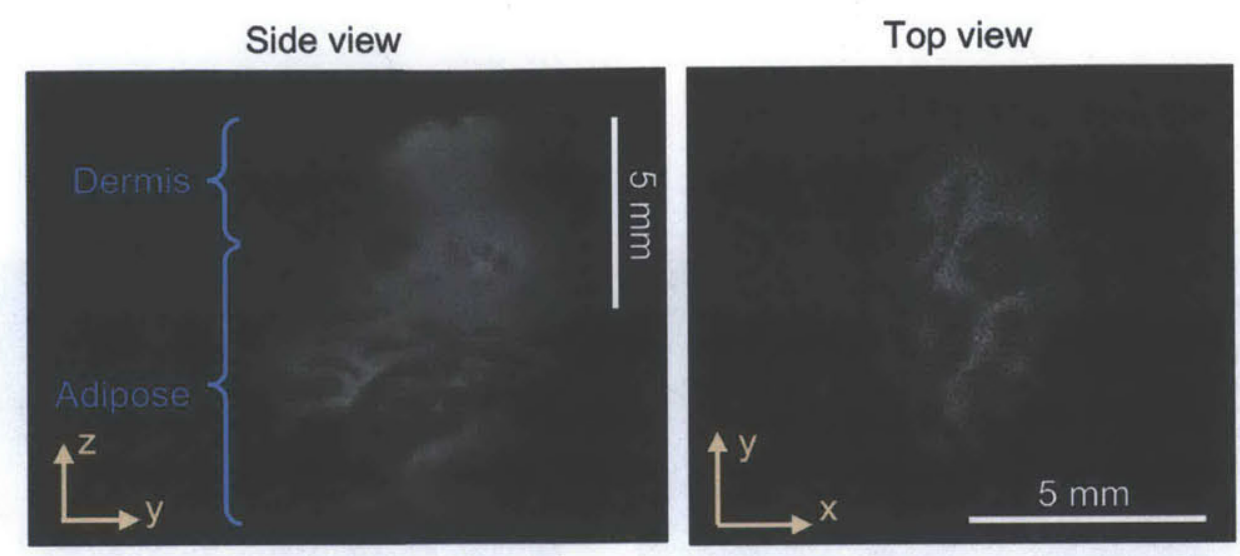

Figure 3-11: Micro-CT images (two-dimensional) of the dispersion pattern for a $100 \mu \mathrm{L}$ injection into the subcutaneous fat layer using the JI.

layer exhibited the sponge-like dispersion pattern for jet injected samples, there was one noticeable difference between jet injected and needle injected samples. In the jet injected samples, there was consistently the appearance of 'streams' of injectate that were branching out of the bulk of the injectate, an example of which is shown in Figure 3-12. These streams have not been visualized before using other imaging techniques. The streams appeared in the subcutaneous fat layer, and it was unclear from the scans whether they were due to the injectate piercing and following a blood vessel. The diameters of the streams ranged from 50 to $150 \mu \mathrm{m}$.

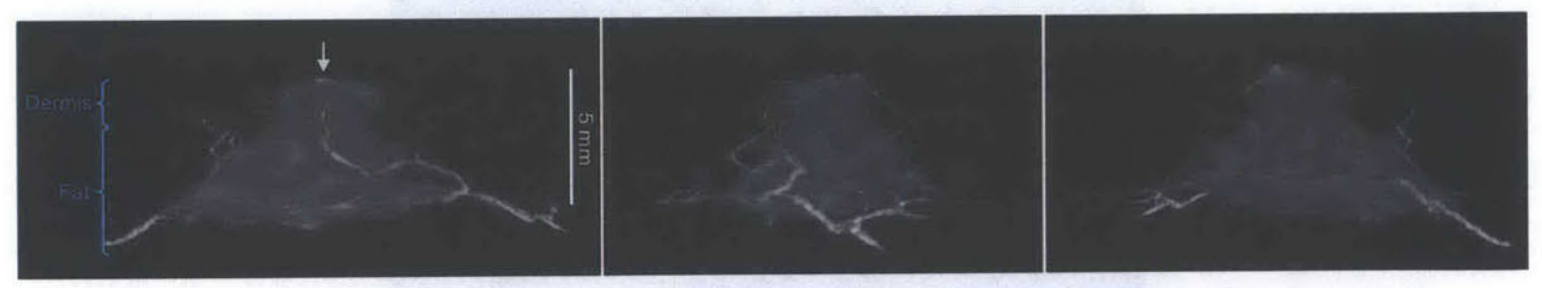

Figure 3-12: 3D reconstructed volume of a jet injected tissue sample. There was the consistent appearance of 'streams' of injectate in the subcutaneous fat layer.

To determine whether the appearance of the injectate streams was due to the injectate following a blood vessel, histology slides were prepared. After micro-CT imaging, the injected samples were frozen and histology slides of $20 \mu \mathrm{m}$ sections were prepared using a cryostat. The slides were fixed in Bouin's solution overnight and then stained with picrosirius red, a collagen-specific dye, for 1 hour. After staining, 
excess dye was washed out with Bouin's solution. Bright field micrographs of the stained sections were taken using a Nikon TE-2000E inverted microscope. An example of a section containing the cross-section of a stream is shown in Figure 3-13. The approximate location of this section with respect to the CT scan is shown in Figure 3-14. The yellow arrow in the figure indicates the stream that was visualized. Figure 3-13 shows that the injectate stream does not follow a vessel; instead it has found weak paths in the septa of the adipose tissue to follow.

\section{Intramuscular injections}

Intramuscular jet injections resulted in the appearance of a dispersion pattern that resembled a 'branched tree.' The histology section in Figure 3-13 shows that during jet injection, the injectate preferentially travels along the perimysium that sheathes the muscle fibers. Micro-CT imaging of intramuscular needle injections showed that the injectate also travelled along the perimysium in needle injections, but the dis-

persion pattern was different depending on the injection technique. For example, if the needle was retracted slightly ( $\mathrm{a}$ few $\mathrm{mm}$ ) after insertion but before injection, the dispersion pattern resembled that of jet injections, with the injectate dispersing into both the subcutaneous fat and the muscle (Figure 3-15). However, if the needle was not retracted before injection, the injectate remained in the muscle, and followed the paths set by the perimysium (Figure 3-16).

\section{Effect of following velocity on injectate distribution}

It is well known from studies done on gel analogs that the reduced following velocity, $v_{\text {follow, }}$, will deliver the remaining injectate without further gel damage. To investigate the effect that the magnitude of $v_{\text {follow }}$ will have on the delivery of fluid into layered tissue, micro-CT scans were done of injections performed with $v_{j e t}=175 \mathrm{~m} / \mathrm{s}, t_{j e t}=$ $2 \mathrm{~ms}, \mathrm{Vol}=100 \mu \mathrm{L}$, and $v_{\text {follow }}$ values of $65( \pm 2) \mathrm{m} / \mathrm{s}, 25 \mathrm{~m} / \mathrm{s}$, and $5 \mathrm{~m} / \mathrm{s}$. When $v_{\text {follow }}=5 \mathrm{~m} / \mathrm{s}$, there were $20-30 \mu \mathrm{L}$ of injectate left on the surface of the skin, indicating that $v_{\text {follow }}$ was too slow to deliver the remaining injectate. A MATLAB script was written to calculate the percentage of total injectate delivered to each 


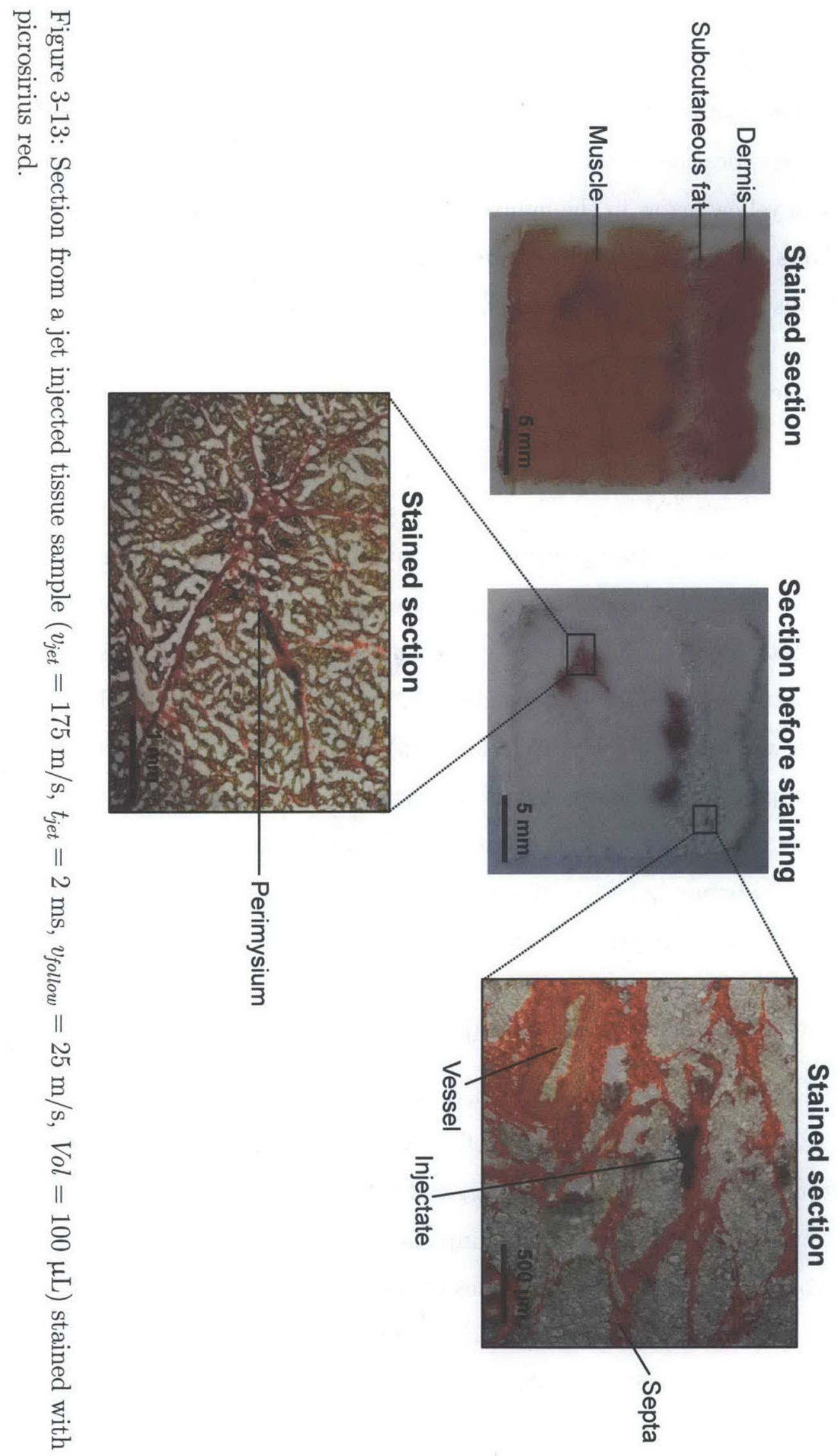




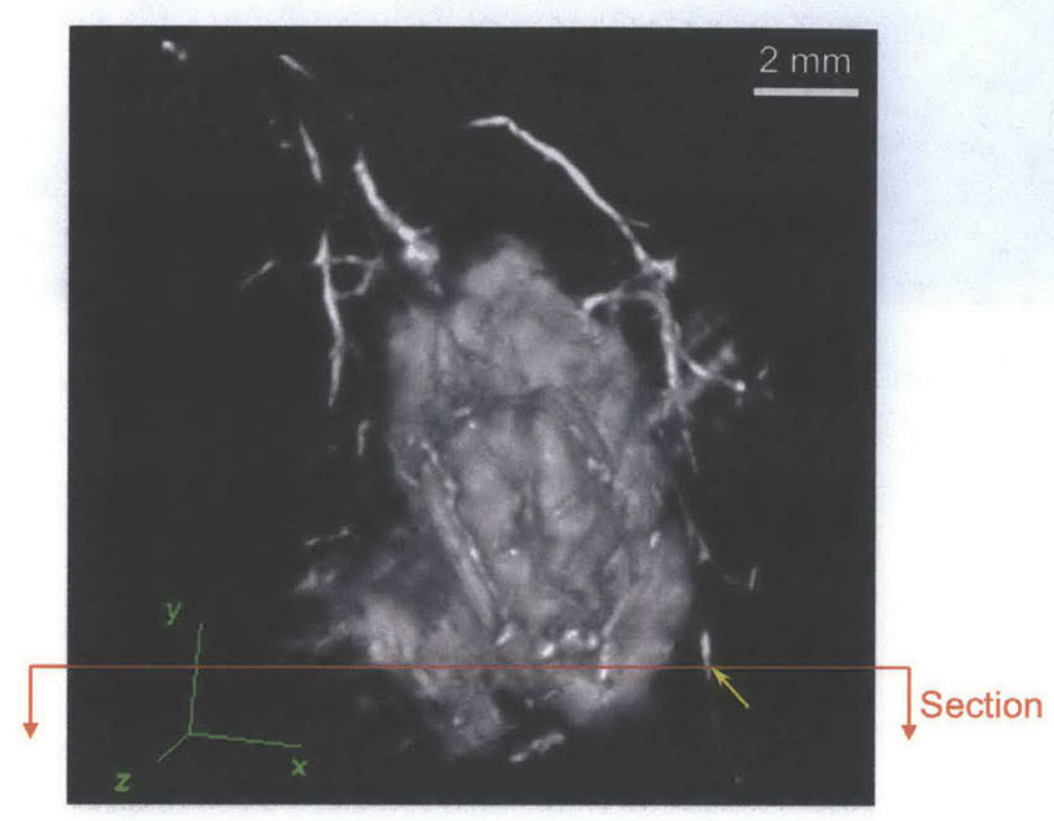

Figure 3-14: Top-down view of 3D reconstructed micro-CT scan of a porcine tissue sample jet injected with $\mathrm{Vol}=100 \mu \mathrm{L}$, at $v_{\text {jet }}=175 \mathrm{~m} / \mathrm{s}, t_{\text {jet }}=2 \mathrm{~ms}$, and $v_{\text {follow }}=25$ $\mathrm{m} / \mathrm{s}$. The location of the section shown in Figure 3-13 is indicated. The yellow arrow notes the 'stream.'

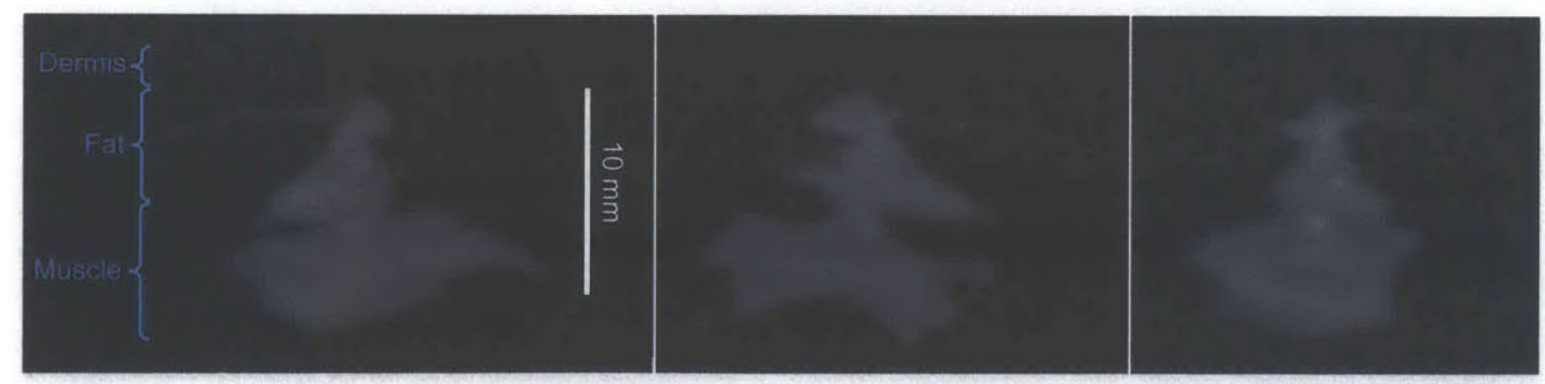

Figure 3-15: 3D reconstructed volume of a micro-CT scan of a $100 \mu \mathrm{L}$ intramuscular needle injection. A 27 gauge $(410 \mu \mathrm{m}$ nominal OD) needle was used. The contrast was injected after the needle was retracted $2 \mathrm{~mm}$. 


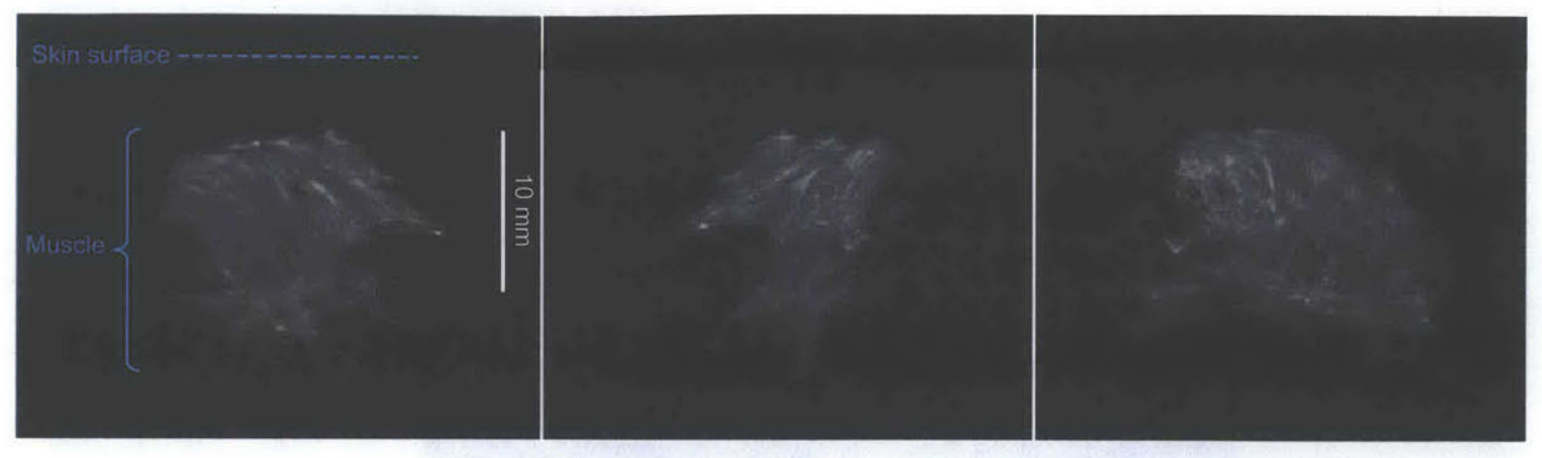

Figure 3-16: 3D reconstructed volume of a micro-CT scan of a $100 \mu \mathrm{L}$ intramuscular needle injection. A 27 gauge $(410 \mu \mathrm{m}$ nominal OD) needle was used. In this case, the needle was not retracted before the contrast was injected.

skin layer from the intensity pixel data outputted by the micro-CT. For skin samples with a thick enough layer of fat such that the injection did not reach the muscle, a higher $v_{\text {follow }}$ resulted in more injectate being delivered to the fat layer. For a typical injection, shown in Figure 3-19, $90 \%$ of the injectate was delivered to the fat when $v_{\text {follow }}=67 \mathrm{~m} / \mathrm{s}$, while only $77 \%$ was delivered to the fat when $v_{\text {follow }}=25 \mathrm{~m} / \mathrm{s}$. If the injectate reached the muscle layer, the slower $v_{\text {follow }}=25 \mathrm{~m} / \mathrm{s}$ resulted in more of the injectate delivered to the fat than the muscle. A typical injection, shown in Figure 3-20, had $67 \%$ delivered to the fat. On the other hand, injecting with the faster $v_{\text {follow }}=63 \mathrm{~m} / \mathrm{s}$ resulted in more of the injectate delivered to the muscle. A typical injection, shown in Figure 3-20, had $59 \%$ of the injectate delivered to the muscle. The slower $v_{\text {follow }}$ resulted in more injectate delivered to the fat because the velocity was not high enough to drive the injectate deep into the muscle. Instead, the injectate was delivered to the fat which is less resistant to fluid flow than the muscle. Thus, the following velocity will determine to which layer the bulk of the injectate will be delivered. 


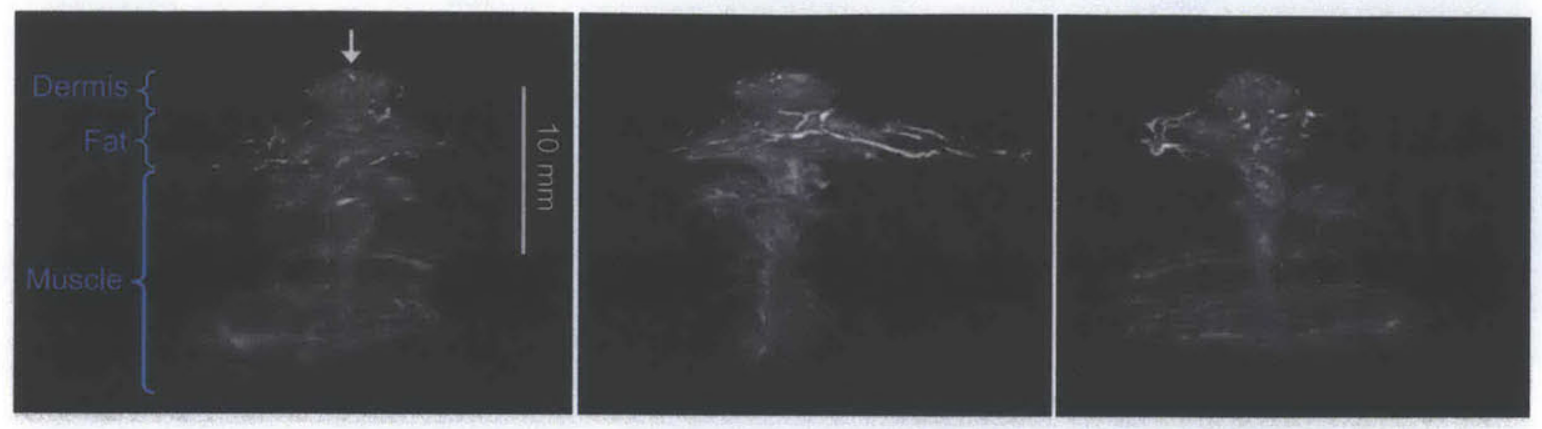

Figure 3-17: 3D reconstructed volume of a micro-CT scan of an intramuscular jet injection, where $v_{\text {follow }}=25 \mathrm{~m} / \mathrm{s}$. The white arrow denotes the injection site.

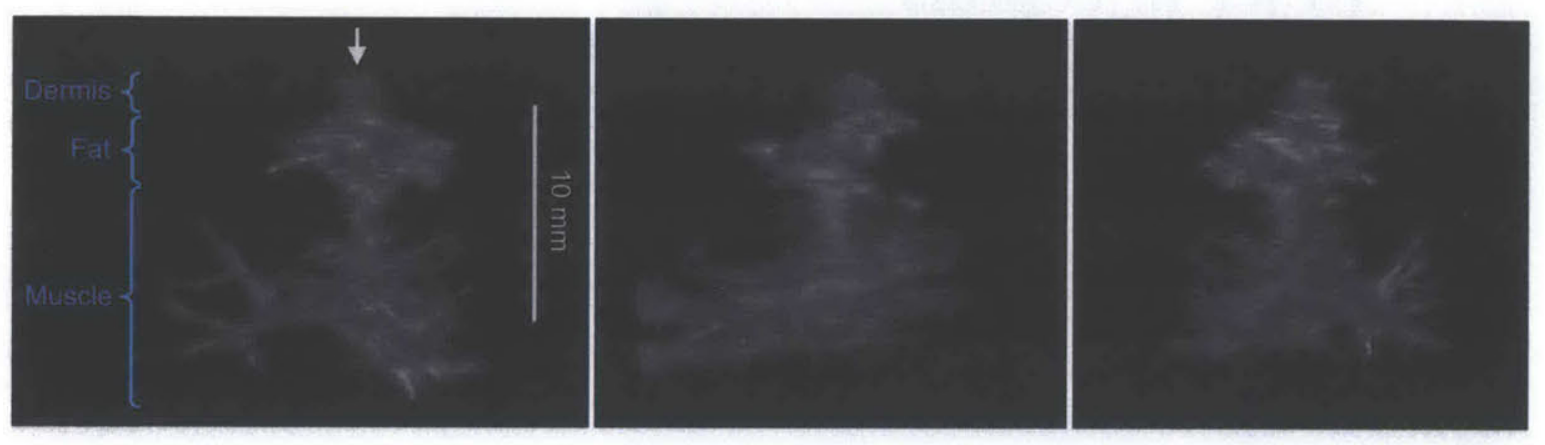

Figure 3-18: 3D reconstructed volume of a micro-CT scan of an intramuscular jet injection, where $v_{\text {follow }}=63 \mathrm{~m} / \mathrm{s}$. The white arrow denotes the injection site.
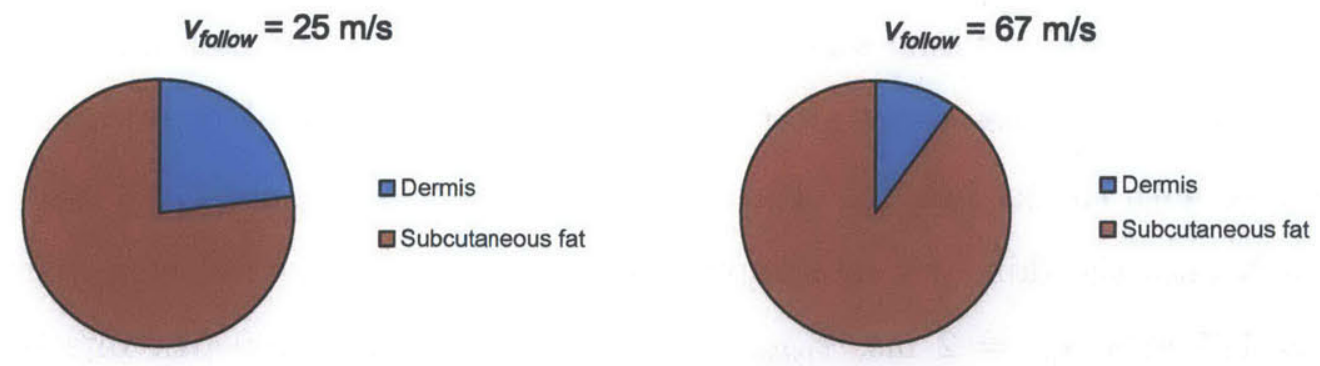

Figure 3-19: Percentage of injectate delivered to each skin layer for different values of $v_{\text {follow }}$. Injections were performed with $v_{\text {jet }}=175 \mathrm{~m} / \mathrm{s}, t_{\text {jet }}=2 \mathrm{~ms}$, and $\mathrm{Vol}=100$ $\mu \mathrm{L}$. In these cases, the injectate did not reach the muscle layer. 

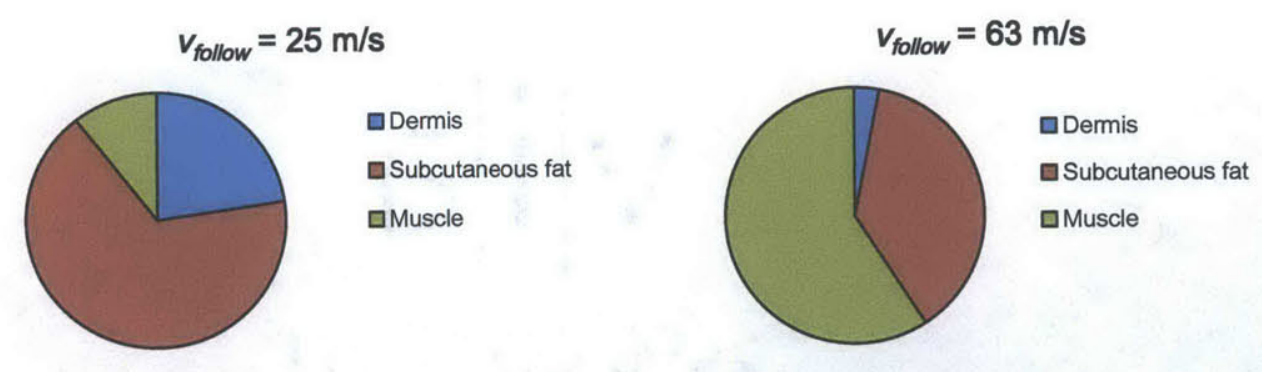

Figure 3-20: Percentage of injectate delivered to each skin layer for different values of $v_{\text {follow }}$ for intramuscular injections. Injections were performed with $v_{\text {jet }}=175 \mathrm{~m} / \mathrm{s}$, $t_{j e t}=2 \mathrm{~ms}$, and $\mathrm{Vol}=100 \mu \mathrm{L}$.

\subsection{Histological examination of tissue damage due to jet injections}

The micro-CT study investigated the injectate dispersion patterns in tissue. To investigate the tissue damage resulting from jet injections, a histology study was conducted. From studies done on gel analogs, it was hypothesized that during the high velocity phase of the injection, a puncture hole with a diameter comparable to the size of the ampoule orifice $(\sim 200 \mu \mathrm{m})$ is created in the tissue. The purpose of this study was to compare the size of the puncture hole created by a 27 gauge ( $410 \mu \mathrm{m}$ nominal OD) needle and that created by the jet injector.

\subsubsection{Experimental Methods}

Post-mortem porcine tissue was obtained and prepared by the methods described in Section 3.2.3. Samples were injected with either a 27 gauge (410 $\mu \mathrm{m}$ nominal OD) needle or with the jet injector. For each jet injection, the JI applied a contact force of $0.8 \mathrm{~N}$ onto the skin. Tissue samples were injected with the following parameters: $v_{\text {jet }}=175 \mathrm{~m} / \mathrm{s}, t_{\text {jet }}=2 \mathrm{~ms}, v_{\text {follow }}=50 \mathrm{~m} / \mathrm{s}$, and $V o l=100 \mu \mathrm{L}$. Injected tissue samples were flash frozen and histology slides containing $20 \mu \mathrm{m}$ sections stained with picrosirius red were prepared with the procedure described in Section 3.3.2. The sections were cut parallel to the skin surface. Bright field micrographs of the stained sections were taken using a light microscope. 


\subsubsection{Results}

Injections performed with a 27 gauge $(410 \mu \mathrm{m}$ nominal OD) needle resulted in puncture holes that were on average $564 \mu \mathrm{m}$ in diameter (with a standard deviation of 94 $\mu \mathrm{m})$. On the other hand, puncture holes created by jet injections were quite different. It was originally expected that there would be a single puncture hole on the order of $200 \mu \mathrm{m}$ (the approximate diameter of the ampoule orifice) to the muscle. However, there was a shallow puncture hole $100 \mu \mathrm{m}$ in diameter within the first $500 \mu \mathrm{m}$ of depth (Figure 3-21a). Deeper in the dermis, there was no single erosion hole but several small penetration holes (Figure $3-21 \mathrm{c}, \mathrm{d}$ ). This is because the jet diverges as the fluid maneuvers around the tough collagen fibers in the dermis, sometimes breaking fibers in the process. Once the jet reached the muscle, since muscle fibers are easier to break than the collagen in the dermis, a large $(400-600 \mu \mathrm{m})$ penetration hole was formed (Figure 3-21d).

When comparing the collagen damage in the dermis caused by the dispersion of the injectate in both needle and jet injections, the collagen fibers had been stretched out and broken (Figure 3-22). This damage may correlate to pain, and is a topic to be investigated in the future. The damage done to the collagen during jet injection results in effectively creating larger pores in the dermis. This information will be used in the FEM model developed in Chapter 5.

\subsection{Summary}

This chapter explored the effect of different injection parameters $\left(v_{j e t}, V o l\right.$, and contact force) on dispersion depth in post-mortem porcine skin. Along with dispersion depth measurements, a micro-CT imaging study was performed to investigate the difference in dispersion patterns when injecting with a needle or with the jet injector. The micro-CT imaging study found that the injectate spread in the subcutaneous fat is similar for a needle and jet injection. It also found that $v_{\text {follow }}$ will determine to which layers the remaining injectate will be delivered. A histological examination of post-mortem skin after needle and jet injections found that while a 27 gauge (410 

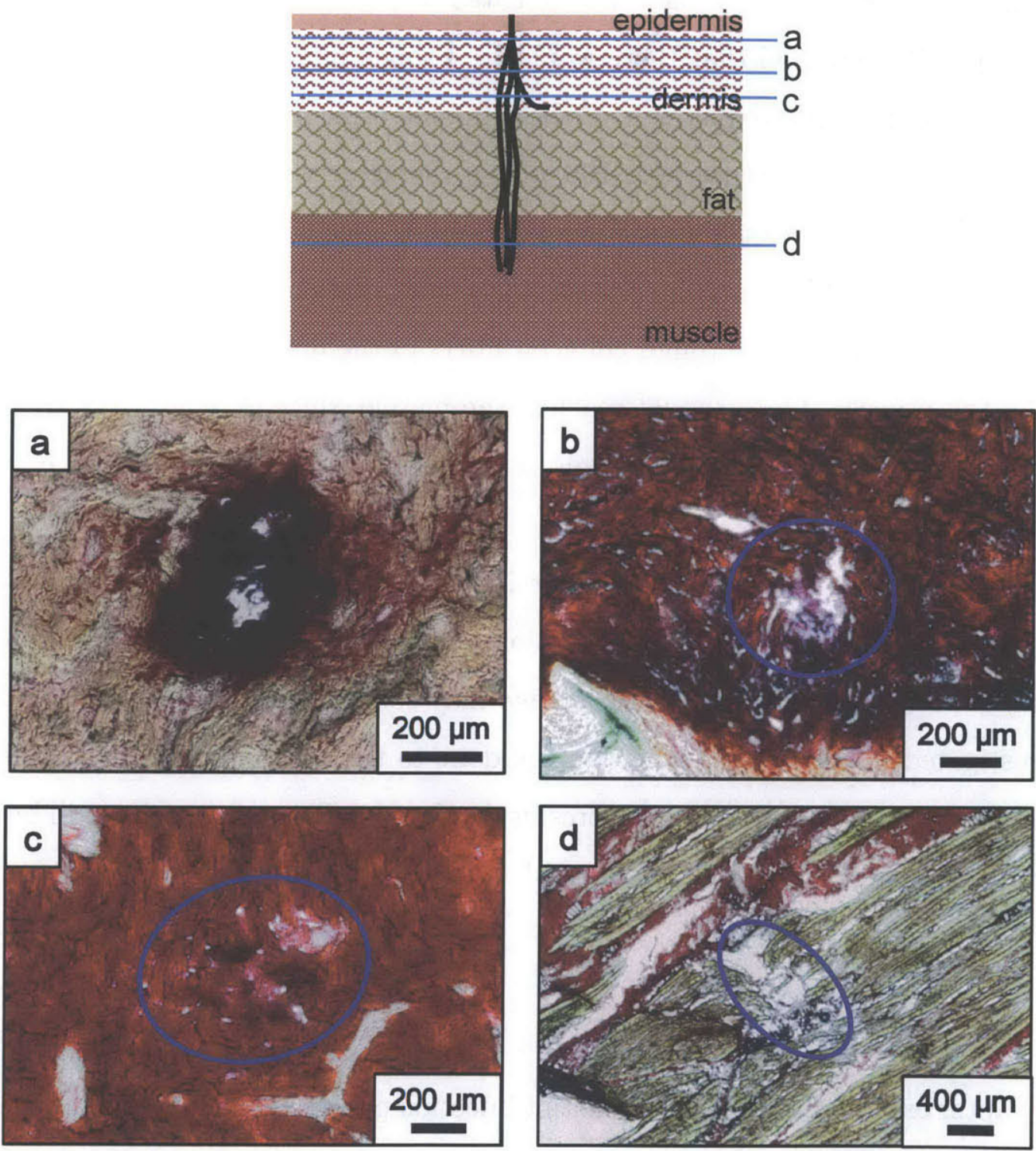

Figure 3-21: Micrographs of $20 \mu \mathrm{m}$ thick cryotomed tissue sections stained with picrosirius red. The sections show the damage resulting from the puncture of the tissue by the jet. The tissue sample was jet injected with the following parameters: $v_{\text {jet }}=175 \mathrm{~m} / \mathrm{s}, t_{\text {jet }}=2 \mathrm{~ms}, v_{\text {follow }}=50 \mathrm{~m} / \mathrm{s}$, and $\mathrm{Vol}=100 \mu \mathrm{L}$. 


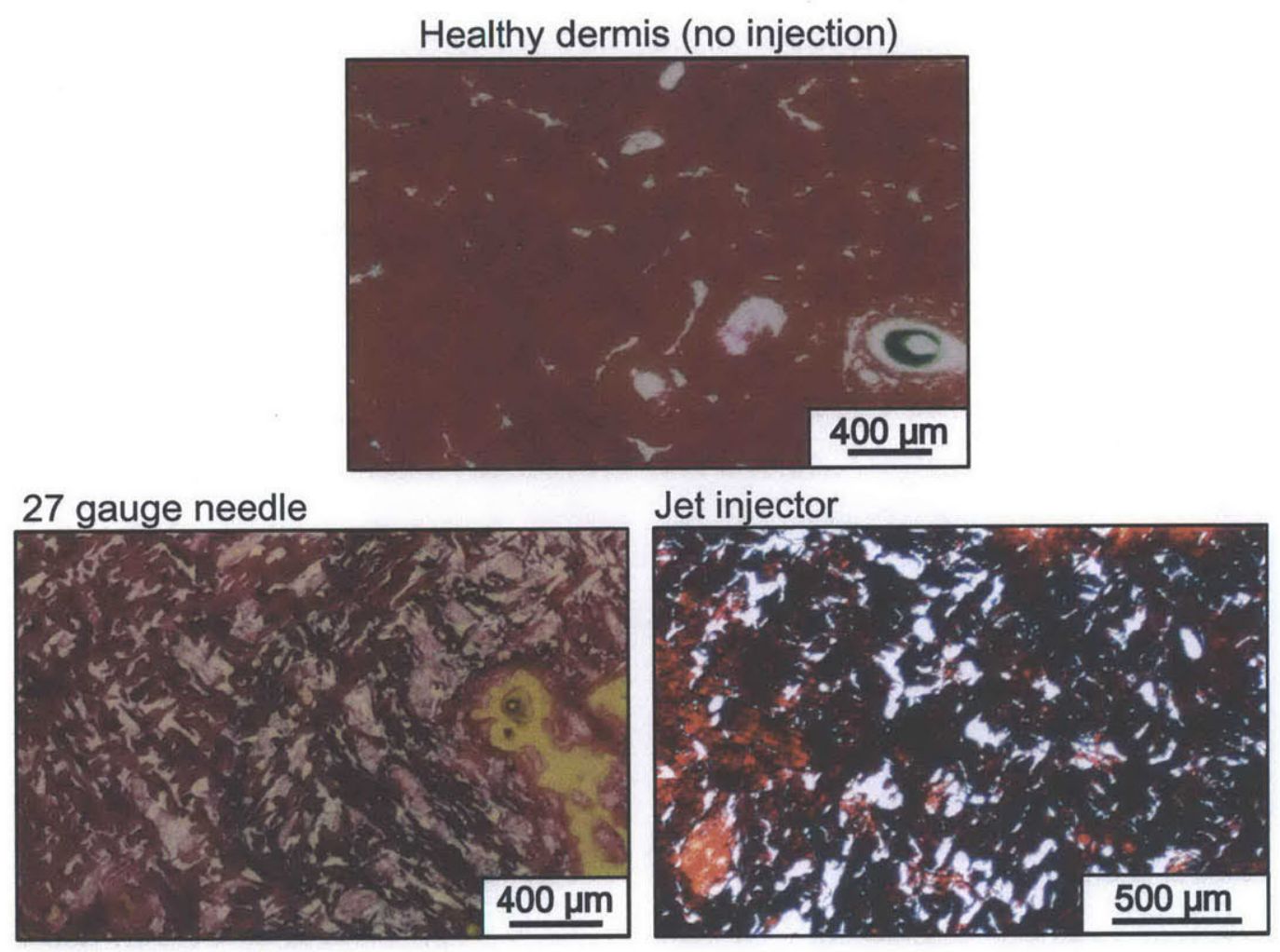

Figure 3-22: Micrographs of $20 \mu \mathrm{m}$ thick cryotomed tissue sections stained with picrosirius red. The sections show the damage resulting from the dispersion of the injectate volume into the tissue, from injections performed with a 27 gauge (410 $\mu \mathrm{m}$ nominal OD) needle and the JI. 
$\mu \mathrm{m}$ nominal OD) needle will create a puncture hole that is $440-700 \mu \mathrm{m}$ in diameter, jet injection will not create a single puncture hole. Instead, jet injection will create multiple smaller holes in the dermis, as the jet maneuvers around the collagenous network. 


\section{Chapter 4}

\section{High-speed X-ray imaging studies}

All of the previous work investigating the effect of jet injections on tissue have been based on observations of the dispersion patterns and tissue damage post injection. High speed images taken of jet injections done into clear tissue analogs (consisting of either polyacrylamide or agarose gel) have elucidated the roles of jet parameters such as $v_{j e t}$ and $t_{j e t}$, but gel analogs are a rather simplistic tissue model. To validate our inferences about the roles of the different jet parameters, we must image the penetration and subsequent dispersion of the liquid jet into tissue at frame rates high enough to distinguish between the penetration phase and the dispersion phase. The opacity of tissue, however, renders visualization of the injection to be challenging. While there are numerous chemical treatments in existence that improve the transmission of light through tissue for enhanced imaging, these treatments cross link the collagen in tissue, thereby changing the mechanical properties [44, 45]. High-speed X-ray imaging offers up a promising method of studying the dispersion of a radiopaque dye through tissue in real-time.

\subsection{Device development}

A typical X-ray imaging system consists of an X-ray source that produces the X-rays, a scintillator plate which then converts the incident X-rays to visible light, and a CMOS or CCD image sensor. The object of interest is placed between the X-ray 
source and the scintillator, and X-rays are either absorbed or pass through depending on the material. The imaging system may include an image intensifier tube between the scintillator plate and the image sensor to increase the intensity of light hitting the image sensor. The scintillator can be directly coupled to the image intensifier tube via a fiber optic coupling or can be optically coupled with a lens. Similarly, the image intensifier can be coupled to the image sensor with a fiber optic coupling or lens.

\subsubsection{Prior Art}

The X-ray Reconstruction of Moving Morphology (XROMM) project at Brown University developed two high-speed X-ray imaging systems [46, 47]. The first consists of two refurbished mobile C-arm fluoroscopes retrofitted with image intensifiers and high-speed video cameras and is capable of imaging volumes about the size of a standard soccer ball. The second system is contained in a custom-built X-ray room and consists of two Varian model G-1086 x-ray tubes, two pulsed X-ray generators, two image intensifiers, and two high-speed cameras, and was built to image larger subjects. Both systems are able to image at up to $1000 \mathrm{fps}$. Higher frame rates are not possible since the decay time of the scintillator (P43) is $1 \mathrm{~ms}$.

Radiation Monitoring Devices (RMD), Inc., manufactures a custom high-speed Xray camera that is capable of imaging up to 2,000 fps at a resolution of $1024 \times 1024$ pixels. The camera consists of a scintillation screen, an image intensifier, a fiber optic taper, and a high speed camera. The cost of the camera is approximately $\$ 500,000$.

The existing X-ray imaging system in the BioInstrumentation Lab, shown in Figure 4-1, was assembled by a graduate student in 2006 [48]. The system consisted of a source (Source-Ray Inc., SB-80-500) that was capable of outputting a maximum of 80 $\mathrm{kVp}$ with a current of $500 \mu \mathrm{A}$, and an X-ray digital camera (Hamamatsu, C4742-56) capable of recording video at $9 \mathrm{fps}$ at full $1344 \times 1024$ resolution $(30 \mathrm{fps}$ at reduced $1280 \times 1024$ resolution). The source and camera were placed inside a lead enclosure, and the entire system was approved for use by the MIT Environment Health

and Safety (EHS) office. To modify the existing system for high-speed imaging, the Hamamatsu camera needed to be replaced. Because a high-speed X-ray camera from 
RMD, Inc. would cost around $\$ 500,000$, we opted to build our own in the BioInstrumentation Lab.

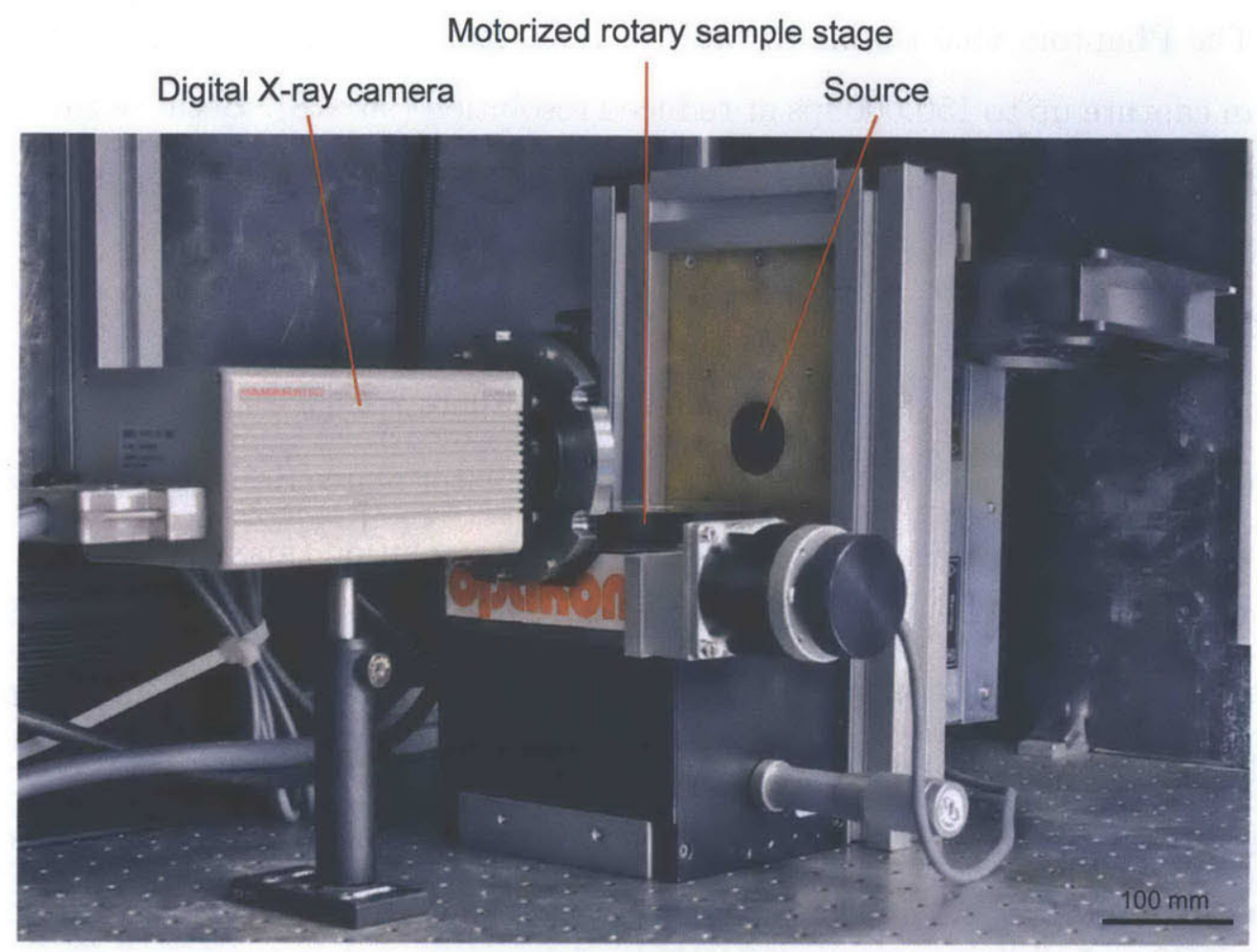

Figure 4-1: Previous X-ray imaging setup. Photo by Dr. Adam Wahab.

\subsubsection{Functional requirements}

Because the primary purpose of the high-speed X-ray imaging system was to image jet injections that occurred in $50 \mathrm{~ms}$ or less, and because the puncture phase is estimated to occur in the first $10 \mathrm{~ms}$, the system needed to have a capture rate of at least 500 fps. The active imaging area needed to be at least $10 \mathrm{~mm} \times 10 \mathrm{~mm}$ to capture the formation of the puncture hole (Section 3.2.4 showed that an intramuscular injection with $v_{\text {jet }}=175 \mathrm{~m} / \mathrm{s}, t_{\text {jet }}=2 \mathrm{~ms}, v_{\text {follow }}=50 \mathrm{~m} / \mathrm{s}$, and $\mathrm{Vol}=100 \mu \mathrm{L}$ will result in dispersion depths of $12 \pm 2 \mathrm{~mm}$ ). The resolution needed to be $10 \mu \mathrm{m}$ to image the penetrating jet with sufficient pixels. 


\subsubsection{Component selection}

The BioInstrumentation Lab already owned a high-speed digital camera, a Phantom v9.0. The Phantom v9.0 is able to capture $1,000 \mathrm{fps}$ at full resolution $(1632 \times 1200)$ and can capture up to $150,000 \mathrm{fps}$ at reduced resolution $(96 \times 8)$. Because the capture rate at full resolution was enough to satisfy the functional requirements, and because the cost of a new high-speed camera was well over $\$ 100,000$, it was decided to use the Phantom v9.0 in the high-speed X-ray setup.

The high-speed setup required an X-ray scintillator with a fast decay time. A cesium iodide doped with thallium (CsI(Tl)) scintillator plate (Hamamatsu, J6671-01) was selected due to its fast decay time $(1 \mu \mathrm{s})$, high resolution (maximum resolution of $35 \mathrm{lp} / \mathrm{mm}$ ), and bright light output (54 photons $/ \mathrm{keV}$ with the maximum of the broad emission at $550 \mathrm{~nm}$ ). However, although CsI(TI) scintillators are one of the brightest scintillators known, the light output was still not high enough to be detected by the Phantom v9.0 at the short exposure times required to capture images at frame rates higher than $100 \mathrm{fps}$. An image intensifier was deemed to be the most cost-effective solution for amplifying the light output of the scintillator. Image intensifiers are commonly used in low-light applications, such as night vision, fluorescence imaging, and astronomy.

A custom-built image intensifier was purchased from Photek Limited (part number MCP125/F/S20/P46/GL). The image intensifier had a Generation II S20 photocathode (which has a wide spectral response from the ultraviolet to near infrared region), a single microchannel plate (with a maximum gain of 4,800), and a P46 phosphor screen (with a decay time of $300 \mathrm{~ns}$ ). The resolution of the output was $40-45 \mathrm{lp} / \mathrm{mm}$.

A lens to optically couple the high-speed camera to the output of the image intensifier was selected rather than using a fiber optic coupling. The primary reason for choosing a lens over a fiber optic coupling was that a fiber optic coupling would need to be permanently bonded to the CMOS, rendering the camera unusable for other projects. Lenses also provide several advantages over using fiber optics: the optical resolution is maintained, there is no distortion of the image from the fiber 
bundle, and there are no honeycomb patterns in the image. The main disadvantage of using a lens is the loss of emitted light from the image intensifier due to the mounting distance between the lens and the image intensifier output. Using a fiber optic coupling would result in much more efficient light transmission to the Phantom v9.0 CMOS detector, and would require a lower gain from the image intensifier.

The scintillator plate was coupled to the input window of the image intensifier with an index-matching silicone optical grease, Visilox V-788. The scintillator was mated to the image intensifier using a custom mount machined out of aluminum. To focus the high-speed camera, the camera was mounted onto a linear stepper motor stage which was controlled by a Compumotor Zeta micro-stepping drive (Parker Motion). Pulse signals were sent to the micro-stepping drive via a Labview program and a USB-6216 data acquisition module (National Instruments). A light-blocking tube was placed between the lens and the image intensifier to keep out unwanted light. The complete high-speed X-ray imaging setup is shown in Figure 4-2.

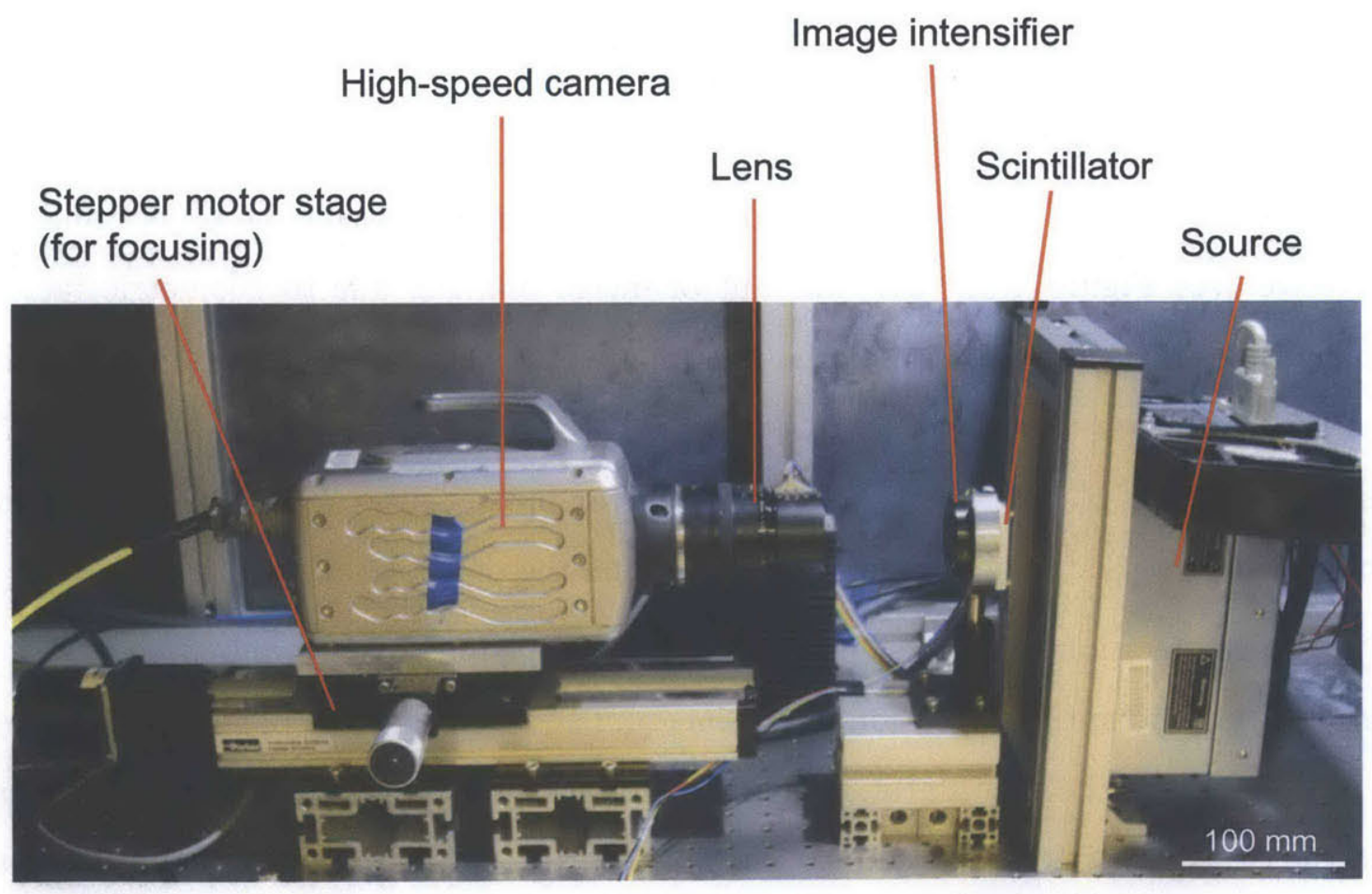

Figure 4-2: The high-speed X-ray imaging setup. The light-blocking tube is not shown. 


\subsubsection{Characterization}

There are three main factors that can cause loss in resolution and image blurring in an X-ray imaging system: motion unsharpness, system unsharpness, and geometric unsharpness. Motion unsharpness is caused by the movement of the object (such as a patient), and can be minimized by keeping the exposure time short. System unsharpness is caused by the finite resolution of the combination of imaging components, such as the scintillator ( 35 line pairs $/ \mathrm{mm}$ ), the lens, and the high-speed camera (maximum resolution of $1632 \times 1200$ pixels). Geometric unsharpness is caused by the geometric factors of the X-ray setup. Since the X-rays are generated over an area rather than a single point, the different paths of the X-rays from the edges of the source to the object will result in less defined edges. The area of varying density at the edges due to geometric unsharpness is called the penumbra. The size of the penumbra, $U g$, is calculated by the following formula:

$$
U g=f \cdot \frac{b}{a}
$$

where $f$ is the focal spot size of the X-ray source, $a$ is the distance from the source to the front surface of the object, and $b$ is the distance between the object and the detector (see Figure 4-3). The amount of image blurring due to geometric factors (or more specifically, the size of the penumbra, $U g$ ) can therefore be minimized by selecting an X-ray source with a smaller focal spot size (the SB-80-500 has a 50 $\mu \mathrm{m}$ focal spot), placing the object and detector further away from the source, and minimizing the distance between the object and the detector.

A high-precision X-ray test pattern (Fluke Biomedical, part number 07-539) was used to determine the resolution of the imaging system. The test pattern was placed $2 \mathrm{~mm}$ in front of the scintillator. The resolution of the imaging system was dependent on the exposure time of the camera, as small patterns were drowned out by noise as the exposure time was decreased. The camera resolution was set at $1280 \times 720$ pixels to allow for imaging at up to 2,000 fps, and the X-ray source was set at a voltage of $40 \mathrm{kVp}$ and a current of $500 \mu \mathrm{A}$. The resolution of the system for different exposure 


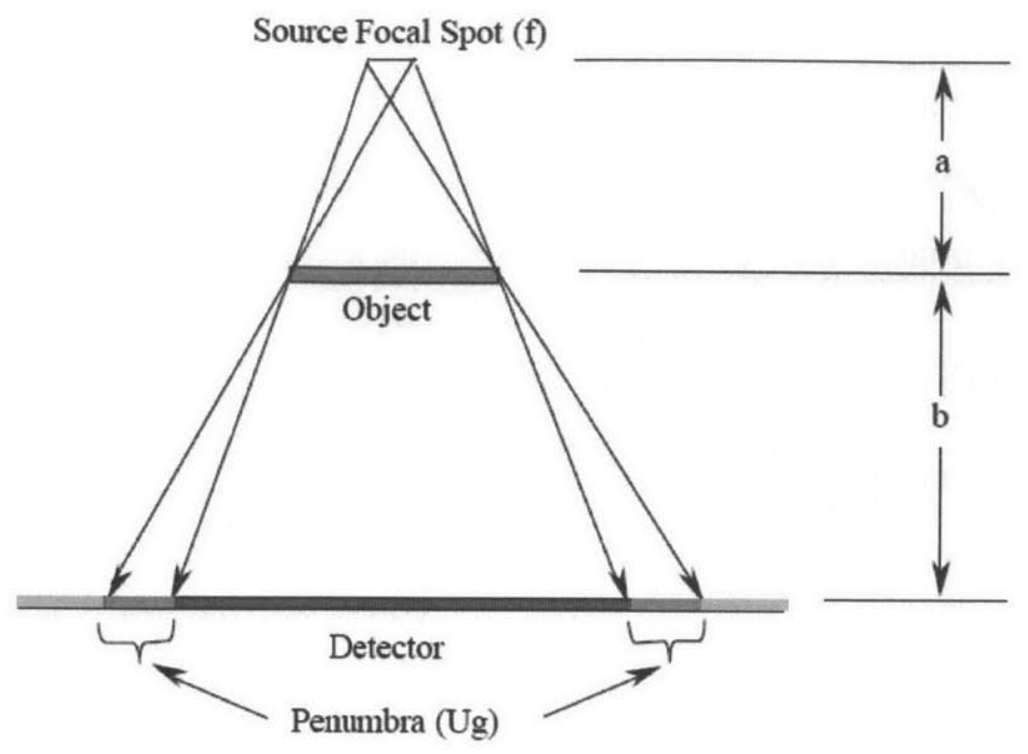

Figure 4-3: Geometric unsharpness, defined by the size of the penumbra $U g$, is dependent on the X-ray source focal-spot size, the distance from the x-ray source to the front surface of the object, and the distance from the object to the detector. Figure from [6].

times and different source to target distances $(a+b)$ is shown in Table 4.1. These resolutions were measured after image processing in MATLAB, which adjusted the brightness and contrast of the image, and then low-pass filtered the noise out of the image using pixelwise adaptive Wiener filtering. At high exposure times (greater than $4900 \mu \mathrm{s})$ the imaging resolution was limited by the resolution of the camera and lens combination. At low exposure times, the imaging resolution was limited by the amount of noise in the image. Placing the detector closer to the source actually resulted in increased resolution, due to the increased light output from the scintillator (from the increased amount of X-rays hitting the scintillator) and subsequently lower amount of noise.

At exposure times less than $490 \mu \mathrm{s}$, the light output from the image intensifier was too low to be detected by the Phantom v9.0. The maximum frame rate of the system was therefore determined to be 2,000 fps (with an exposure time of $490 \mu \mathrm{s}$ ) which exceeded the original functional requirement of $500 \mathrm{fps}$. The active imaging area with the selected lens and resolution combination was 21.5 to $28 \mathrm{~mm} \times 19 \mathrm{~mm}$, which also 
exceeded the original functional requirement. The only specification that was not met was the resolution, since the resolution at the maximum 2,000 fps was found to be $143 \mu \mathrm{m}$. However, the resolution can be greatly improved and the specification can be met by upgrading the components of the imaging system Section 4.1.5.

Table 4.1: Resolution of high-speed X-ray imaging system

\begin{tabular}{|l|l|l|l|l|}
\hline $\begin{array}{l}\text { Exposure } \\
\text { time }(\mu \mathrm{s})\end{array}$ & $\begin{array}{l}\text { Max frame } \\
\text { rate }(\mathrm{fps})\end{array}$ & $\begin{array}{l}\text { Resolution }(\mu \mathrm{m}) \\
(a+b=45 \mathrm{~mm})\end{array}$ & $\begin{array}{l}\text { Resolution }(\mu \mathrm{m}) \\
(a+b=35 \mathrm{~mm})\end{array}$ & $\begin{array}{l}\text { Resolution }(\mu \mathrm{m}) \\
(a+b=30 \mathrm{~mm})\end{array}$ \\
\hline \hline 9,900 & 100 & 42 & 42 & 42 \\
4,900 & 200 & 42 & 42 & 42 \\
1,900 & 500 & 71 & 71 & 71 \\
990 & 1,000 & 100 & 100 & 100 \\
660 & 1,500 & 143 & 125 & 125 \\
580 & 1,700 & 143 & 143 & 143 \\
490 & 2,000 & 167 & 143 & 143 \\
\hline
\end{tabular}

\subsubsection{Future improvements}

The maximum frame rate, the resolution, and the signal to noise ratio can be greatly improved by upgrading the components of the imaging system. A more powerful source that is capable of outputting higher currents will result in brighter images, leading to shorter required exposure times and therefore higher frame rates. The Powermax 1260 manufactured by Source Ray has a maximum power output of $3 \mathrm{~kW}$ and is capable of outputting a voltage range of $40-120 \mathrm{kV}$ with a current range of 0-60 $\mathrm{mA}$ (120 times the maximum current of the SB-80-500). Replacing the source will require modifications to and recertification by the MIT EHS Office of the lead enclosure for the X-ray system. An image intensifier with multiple microchannel plates will also lead to brighter images. An image intensifier can be purchased with up to three microchannel plates, for a maximum gain of $10^{7}$. Additionally, using fiber optic channels in a permanent imaging setup will reduce the amount of light lost by using lens couplings. Finally, upgrading the high speed camera to a peltier-cooled CMOS camera will reduce the amount of noise in the image. The Phantom v1610 
has a sensitivity rating of $64,000 \mathrm{~T}$ for the monochrome sensor (using the ISO 12232 SAT method), which is 122 times that of the Phantom v9.0 (ISO 525T). Upgrading the high speed camera will also enable imaging at higher resolutions at high frame rates.

\subsection{X-ray imaging of needle injections}

Flow patterns for needle injections were imaged with the X-ray imaging system. Two different techniques were examined: injection after needle retraction and injection after no needle retraction. A needle injection system that injected a set volume of fluid at a constant speed into tissue using a 27 gauge $(410 \mu \mathrm{m}$ nominal OD) needle was constructed. The system consisted of two linear stepper motors (Haydon Switch and Instrument 20000 series): one lowered the needle to pierce the tissue, and the other pushed the plunger to inject the tissue. A Geckodrive G251X Digital Step Drive controlled each motor. A cRIO system instead of the USB-6216 data acquisition module was used to send pulse signals fast enough to drive the motors at speeds up to $10 \mathrm{~mm} / \mathrm{s}$. The needle injection system is shown in Figure 4-4.

The needle injection system was used to inject $100 \mu \mathrm{L}$ of radiopaque dye (AuroVist$15 \mathrm{~nm}$, Nanoprobes, Inc.) at a flow rate of $100 \mu \mathrm{L} / \mathrm{s}$ into the muscle of post-mortem

porcine skin. High-speed X-ray videos were taken with a source voltage of $60 \mathrm{kVp}$, tube current of $500 \mathrm{~mA}$, at $500 \mathrm{fps}$ with an exposure time of $1900 \mu \mathrm{s}$. Videos were taken of injections performed without retracting the needle (frames from typical videos are shown in Figure 4-5a and 4-5b), and of injections performed after retracting the needle approximately $2 \mathrm{~mm}$ (frames from a typical video are shown in Figure 4-6).

Figure 4-5a shows a typical flow pattern of the radiopaque dye when an injection was performed without retracting the needle. The tissue was oriented such that the muscle fibers were parallel to the lateral plane. It is evident from the upward flow at the beginning of the injection (for $t<400 \mathrm{~ms}$, where $t$ is time after the start of injection) that the tissue underneath the needle had been compressed and impeded 


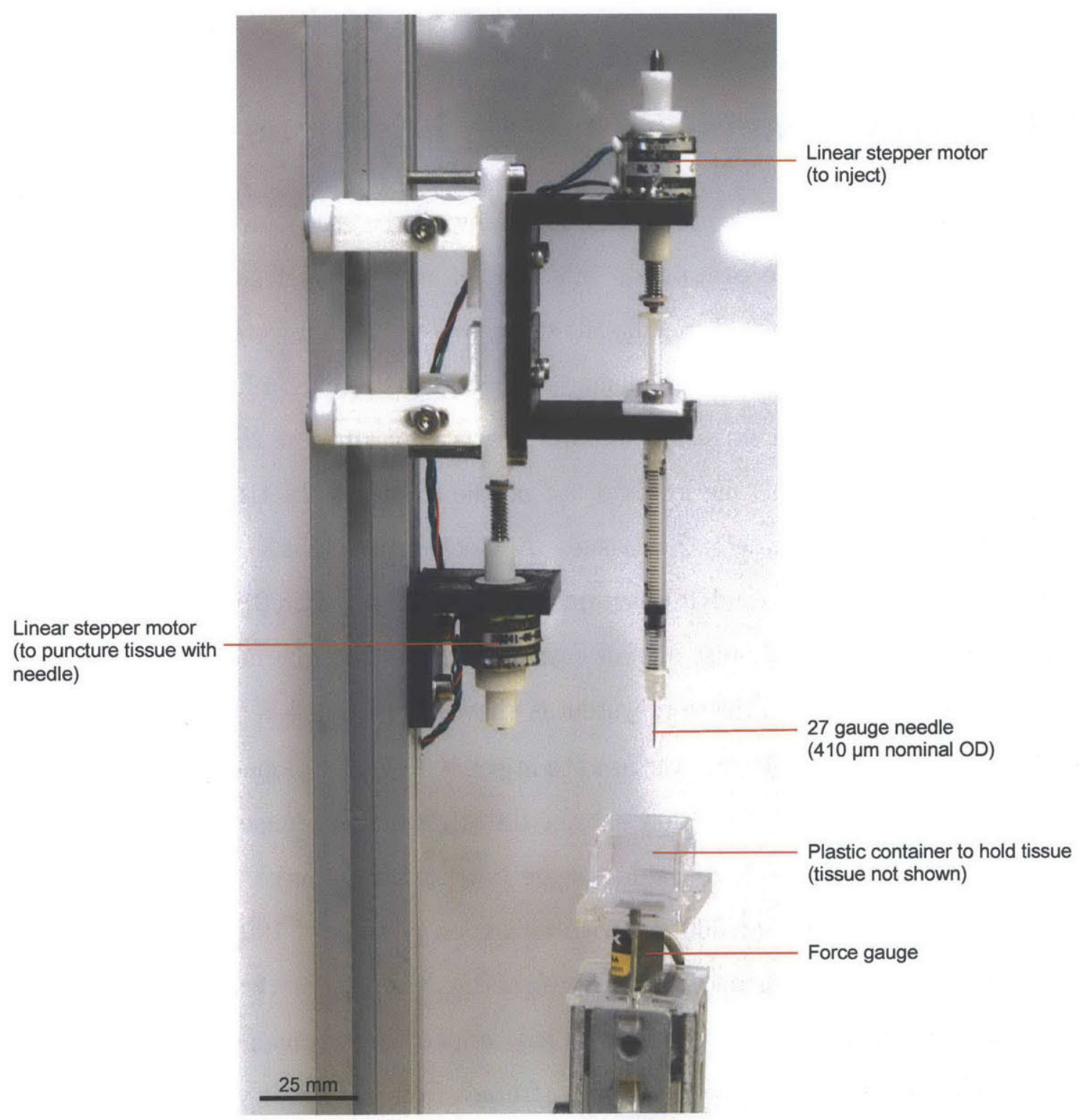

Figure 4-4: The needle injection system used to inject a set volume of fluid into post-mortem porcine skin. 
downward flow, with all of the injectate ending up above the end of the needle. Towards the end of the injection $(t>400 \mathrm{~ms}$ ), the injectate followed the paths set by the muscle fibers and most of the fluid flow occurred parallel to the muscle fibers. In a separate injection, the tissue was oriented such that the muscle fibers were parallel to the sagittal plane (Figure 4-5b). Again, the injection was performed without retracting the needle and all of the injectate ended up above the end of the needle. The majority of the flow was upward at the beginning of the injection $(t<400 \mathrm{~ms})$. Towards the end of the injection $(t>400 \mathrm{~ms})$, fluid flow was parallel to the muscle fibers, indicated by the increasing intensity of the bolus. A 3D volume of an injection performed without retracting the needle can be seen in Figure 3-16.

Figure 4-6 shows a typical flow pattern of the radiopaque dye when an injection was performed after retracting the needle approximately $2 \mathrm{~mm}$. In the beginning of the injection $(t<400 \mathrm{~ms}$ ), the majority of the flow was vertical (both upwards and downwards), as the injectate filled the puncture hole created by the needle. Towards the end of the injection $(t>400 \mathrm{~ms}$ ), the fluid flow was horizontal, as the injection hole became saturated with the injectate. A $3 \mathrm{D}$ volume reconstruction of an injection performed after the needle was retracted can be seen in Figure 3-15.

\subsection{High speed X-ray imaging of jet injections}

To demonstrate that the X-ray imaging system could be used to image the dynamics of injections into tissue, a jet injector device was built and placed inside the X-ray chamber. High-speed videos were taken at 2,000 fps with an exposure time of $490 \mu \mathrm{s}$, with a source voltage of $40 \mathrm{kVp}$ and a tube current of $500 \mu \mathrm{A}$. Frames from a typical jet injection are shown in Figure 4-7b. The corresponding piston velocity for this particular injection is shown in Figure 4-7a. The muscle layer can be distinguished from the dermis/subcutaneous fat layer since it appears darker (more dense). The dermis layer is indistinguishable from the fat layer.

The fluid flow due to needle-free jet injection is remarkably different than that of needle injections. Previous studies have reported that a typical jet injection into 


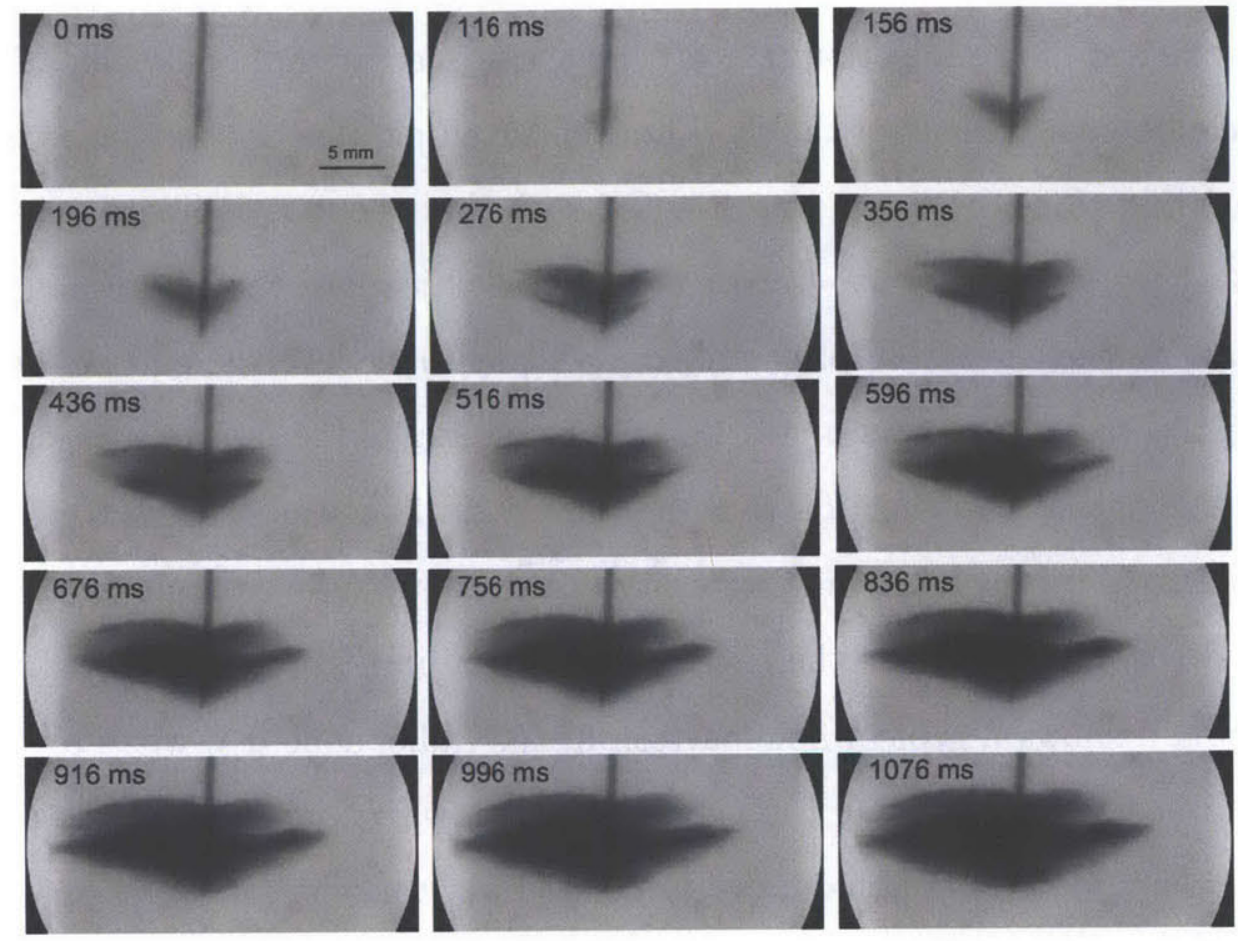

(a) Frames from video where the muscle fibers were oriented parallel to the lateral plane

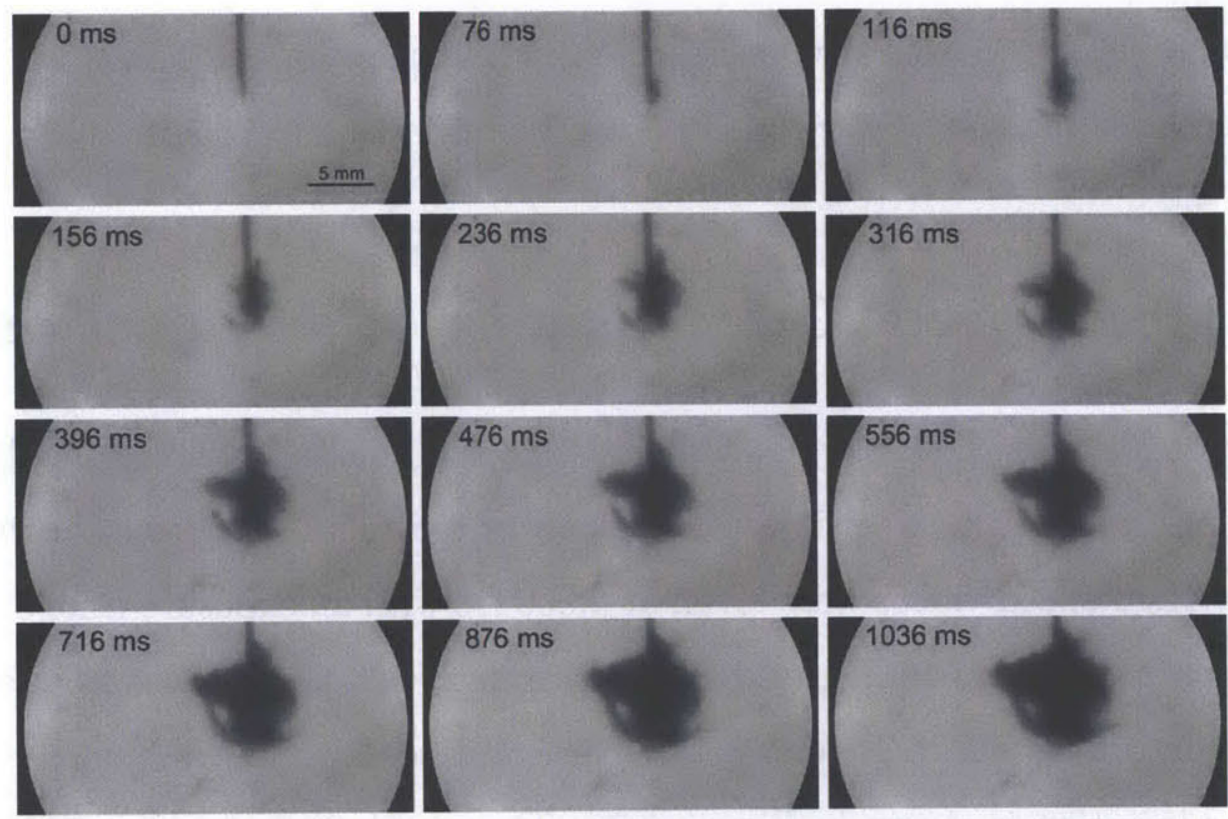

(b) Frames from video where the muscle fibers were oriented parallel to the sagittal plane

Figure 4-5: Frames from X-ray video taken of a $100 \mu \mathrm{L}$ intramuscular needle injection into post-mortem porcine skin. The injections were performed without retracting the needle. 


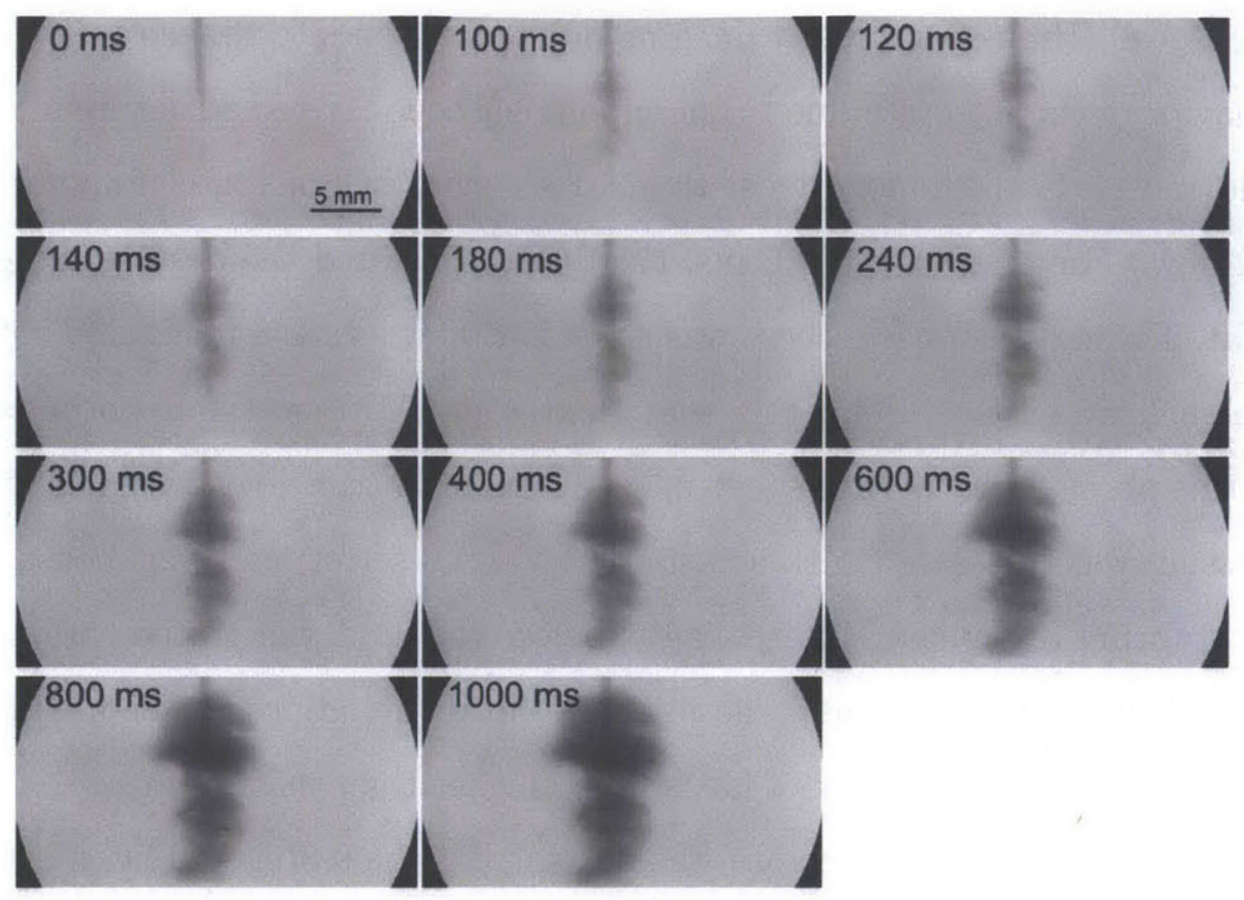

Figure 4-6: Frames from X-ray video taken of a $100 \mu \mathrm{L}$ intramuscular needle injection into post-mortem porcine skin. The injection was performed after retracting the needle approximately $2 \mathrm{~mm}$.

porcine skin will result in delivery of approximately $80 \%$ of the desired volume, with the residual $20 \%$ remaining on the surface of the skin [27]. Until now, it was unclear whether the residual volume left on the skin surface was from the beginning of the injection (fluid that had not been accelerated to the sufficient pressure to pierce the skin surface), or from backflow. Baxter and Mitragoti suggested that fluid that had been delivered at a flow rate too high to be absorbed into the tissue, resulting in fluid flowing back out through the penetration hole [40]. Figure 4-7b shows that the former is true: fluid will collect on the surface of the skin until the injectate has been accelerated to a pressure high enough to breach the skin. The skin was not breached until $t=4.5 \mathrm{~ms}$, when the piston reached a velocity of $0.47 \mathrm{~m} / \mathrm{s}$. From these results, we recommend that the piston be accelerated as fast as possible to minimize the amount of residual injectate left on the surface of the skin.

Furthermore, jet injections into clear gel analogs have suggested that a high initial jet speed is required to puncture the skin and establish penetration depth while a slower following speed delivers the remaining fluid volume without further tissue 
damage $[1,7-8]$. High-speed X-ray imaging of jet injection into tissue reveal the different behavior of the injectate due to the inhomogeneous layered structure of skin. For example, muscle is much more dense than fat and thus will be more of an impediment to fluid flow. For $4 \mathrm{~ms}<t \leq 7.5 \mathrm{~ms}$, the fluid penetrated the dermis and subcutaneous fat. During this phase, there was some backflow as some injectate continues to pool on the skin surface, indicating that Baxter and Mitragotri's hypothesis is valid during this phase. The average speed of hole formation (the downward velocity of the jet in tissue) was calculated with a custom MATLAB script, and was measured to be $0.75 \mathrm{~m} / \mathrm{s}$ during this phase. Interestingly, before the fluid penetrated the underlying muscle $(7.5 \mathrm{~ms}<t \leq 10.5 \mathrm{~ms})$, the injectate filled the adipose layer in the form of a bolus, since the permeability of fat is much higher than that of muscle. The speed of the radial spread of the injectate was measured to be $0.8 \mathrm{~m} / \mathrm{s}$. The injectate then breached the muscle layer $(10.5 \mathrm{~ms}<t \leq 15 \mathrm{~ms})$, at a speed of $1.14 \mathrm{~m} / \mathrm{s}$. We hypothesize that this breaching occurs after pressure has built up in the adipose layer, rendering it more resistant to fluid flow than the muscle. The breaching speed of the muscle is higher than the breaching speed of the dermis/subcutaneous fat layer because the muscle's resistance to puncture is much less than that of the tough dermis. The breaching continued until the piston velocity was lowered to the slower following speed of $0.25 \mathrm{~m} / \mathrm{s}$ (at $t=15 \mathrm{~ms}$ ). Once the piston velocity was lowered, the remaining injectate volume was delivered to the muscle $(t>15 \mathrm{~ms})$. The speed of the radial spread in muscle was $0.12 \mathrm{~m} / \mathrm{s}$.

In Section 3.3.2, it was noted that the injectate dispersion patterns for intramuscular jet injections appeared similar to the patterns for needle injections performed after retracting the needle a few millimeters. The high-speed X-ray imaging of needle and jet injections confirm that while the dispersion patterns may appear similar, the dynamics of the injectate distribution are very different between needle and jet injections. 


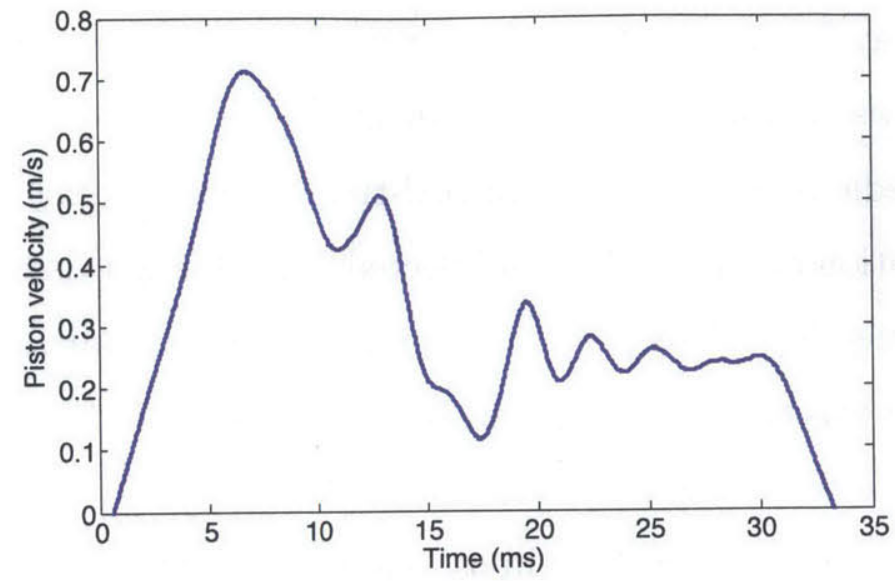

(a) Velocity profile of the piston (piston velocity is proportional to the ejected fluid velocity).
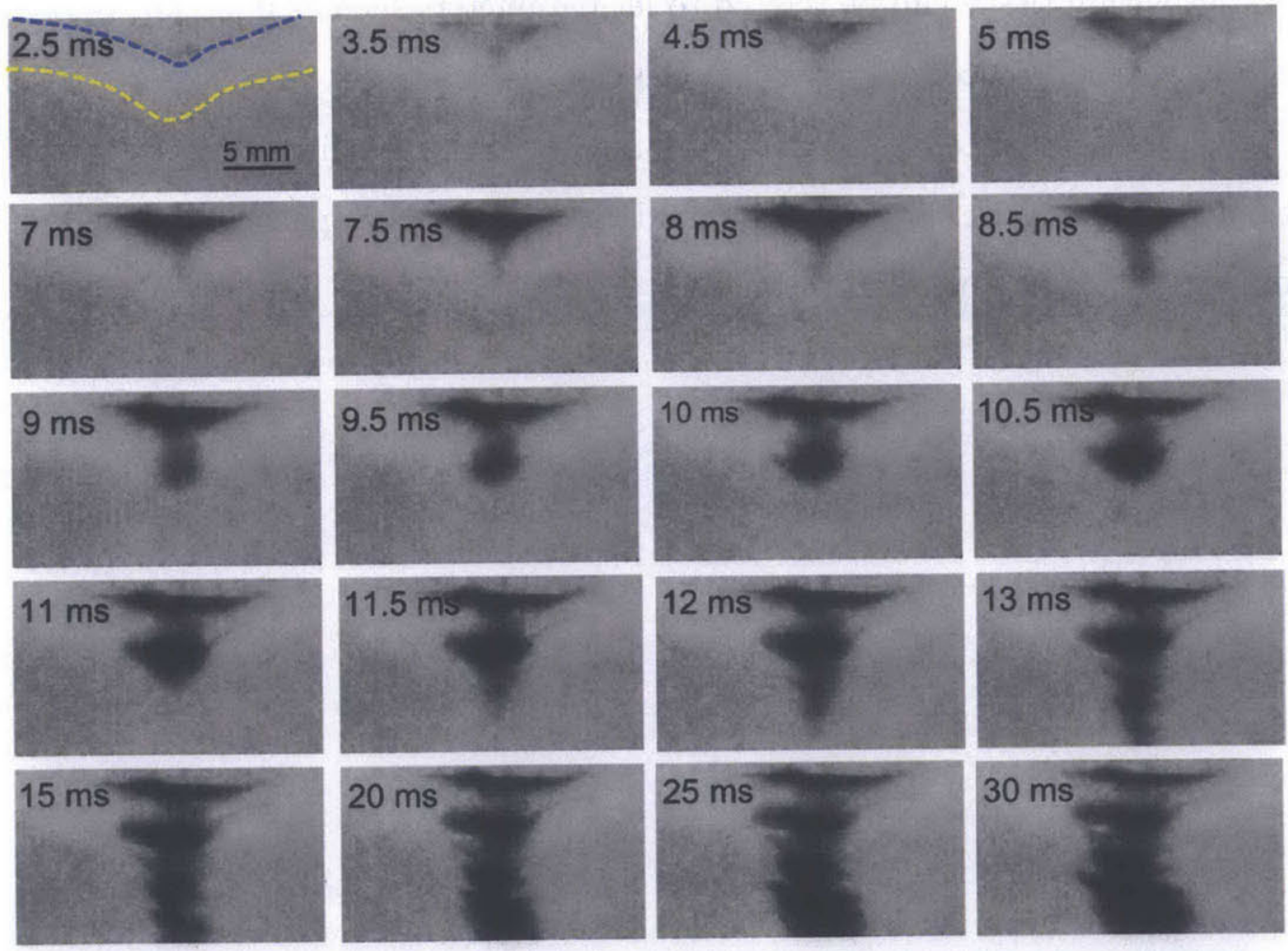

(b) Frames from high-speed X-ray video taken of a $100 \mu \mathrm{L}$ injection into post-mortem porcine tissue. The blue dashed line outlines the skin surface and the yellow dashed line shows the approximate location of the boundary between the subcutaneous fat and the muscle.

Figure 4-7: High-speed X-ray video was taken of a needle-free jet injection at a frame rate of 2,000 fps and an exposure time of $490 \mu \mathrm{s}$. 


\subsection{Summary}

A high-speed X-ray imaging system with a capture rate of up to 2,000 fps was built. This system allows us for the first time to image the flow of fluid through tissue injected by a needle-free jet injector in real-time. This imaging system provides a wealth of information about the flow of injected fluid through tissue. The differences in fluid flow between injections performed with and without partial needle retraction were presented. It was also shown that while jet injection into tissue is similar to that of jet injection into gel analogs, the injectate will preferentially follow paths of least resistance, dispersing through the adipose layer before breaching the muscle layer. Finally, for the first time, the jet velocities within tissue have been measured. The jet velocities within tissue can be related to jet parameters such as injection velocity and injection volume. Since $v_{\text {jet }}$ and $v_{\text {follow }}$ are the theoretical jet velocities at the instant the jet leaves the orifice, a more accurate measurement velocity is the speed of the jet in tissue. Future work can relate the fluid velocity and flow patterns to drug uptake, leading to improved injection techniques. 


\section{Chapter 5}

\section{Modeling of Interstitial Fluid Acquisition Method}

This chapter utilizes Comsol Multiphysics 4.3a to generate a first-order model of the needle-less ISF acquisition process. First, an analytical relationship between tissue swelling and tissue permeability is developed. This relationship is input into Comsol, and the poroelasticity and solute transport modules are used in conjunction to analyze the effects of different injection and extraction parameters on sample acquisition. The purpose of this model was to explain the trends that will be seen during experimentation presented in Chapter 6, and extrapolate for animals of different tissue types.

\subsection{Model setup and assumptions}

For a first-order model of the acquisition process, only the fluid flow and solute transport in the dermis was considered. The underlying layers (subcutaneous fat and muscle) were not considered in this model. 


\subsubsection{Darcy's Law}

Darcy's law gives the constitutive law for fluid flow through a porous medium [49]:

$$
Q=-\frac{k A \nabla P}{\mu},
$$

where $Q$ is the volumetric flow rate, $k$ is the permeability (also referred to as specific hydraulic conductivity, given in $\left.\left[\mathrm{m}^{2}\right]\right), A$ is the cross-sectional area perpendicular to flow, and $\nabla P$ is the pressure gradient. It is commonly used to describe groundwater flow through soil, and is valid only for laminar flow. Experimental data have shown that Darcy's Law is valid for Reynolds numbers, $R e<10$, where $R e$ for porous media flow is given by:

$$
R e=\frac{\rho v D_{p}}{\mu(1-\epsilon)},
$$

where $\rho$ is the density of the fluid, $v$ is the superficial velocity (velocity as if the particles were absent), $D_{p}$ is the average diameter of the particles, $\mu$ is the dynamic viscosity of the fluid, and $\epsilon$ is the fractional void volume $(\epsilon=1-\phi$, where $\phi$ is the fractional solid volume) [50]. In this model, we will look only at the flow occurring in the dermis. First, we assume that most flow is occurring in the reticular layer of the dermis, since it comprises $90 \%$ of the dermal thickness. Then we can approximate $D_{p} \approx 30 \mu \mathrm{m}$ (the thickness of the collagenous bundles in the reticular layer, described in Section 3.1.1). The mean fractional volume of collagen fibers in the dermis has been reported to be $66 \%$ in both the papillary and reticular layers [51,52], so $\epsilon=0.34$. The following values for the viscosity and density of water will be used: $\mu_{\text {water }}=1 \mathrm{mPa} \cdot \mathrm{s}$ and $\rho_{\text {water }}=1000 \mathrm{~kg} / \mathrm{m}^{3}$. Then, the critical fluid velocity at which Darcy's Law begins to degrade is $330 \mathrm{~mm} / \mathrm{s}$. It is worth noting that this velocity is the superficial fluid velocity, which is different than the pore velocity. The velocity of the fluid within the pores is actually much higher than the superficial velocity because the fluid needs to travel around physical barriers, and is given by: $v_{\text {pore }}=v / \epsilon$. The critical pore velocity at which Darcy's Law begins to degrade is thus $970 \mathrm{~mm} / \mathrm{s}$. Because it will be seen from experimentation that the bulk of the fluid is extracted from the tissue 
in a matter of a few seconds (Chapter 6), and the thickness of the dermis is typically 1-4 mm, we can assume that during the extraction process the velocity of the fluid is on the order of $1 \mathrm{~mm} / \mathrm{s}$ which is well below the critical velocity. Thus, Darcy's Law is valid for the extraction phase.

\subsubsection{Resistance to Dermal Interstitial Flow}

An important parameter of Darcy's Law is the material property $k$, the permeability of a porous medium. The resistance to hydraulic flow in the interstitium is caused by the combined interactive effects of three main elements: collagen fibrils, glycosaminoglycans (GAGs) and proteoglycan core proteins (PGPs). While the permeability of simple porous media models (e.g. packed bed of uniform spheres) can be calculated directly, the calculation of dermis permeability is less straightforward. For one, collagen fibrils provide physical resistance to flow, but the collagenous matrix more closely resembles a network of randomly oriented rods than a bed of uniform spheres. Additionally, as described in Section 3.1.1, GAGs (i.e. hyaluronate) bind to tissue fluid rendering the dermis as a gel-like material, providing further resistance to flow. PGPs also provide physical resistance to flow, similar to collagen fibrils. The tortuosity of the routes that fluid must take in the dermis also plays a large role. J. R. Levick explored the relative importance of each of these elements in his seminal paper on the hydraulic conductivity of the interstitium [51]. The following summarizes his derivation and applies his derivation to the calculation of the permeability of the dermis.

As described in Section 3.1.1, collagen in the dermis is organized into fibrous bundles $(0.3-3 \mu \mathrm{m}$ thick in the papillary dermis and 10-40 $\mu \mathrm{m}$ thick in the reticular dermis) [3], which are composed of closely packed thin collagen fibrils. The main types of collagen in the dermis are Type I and Type III collagen. In the papillary dermis, Type I collagen exists in proportions of $87 \%$ and Type III collagen exists in proportions of $13 \%$. In the reticular dermis, $91 \%$ of collagen is Type I while the remaining $9 \%$ is Type III. Type I collagen fibrils are $60-100 \mathrm{~nm}$ in diameter while the finer Type III collagen fibrils are $20-40 \mathrm{~nm}$ in diameter [53]. Since the reticular 
dermis accounts for $90 \%$ of the dermal thickness, and the majority of the collagen in the reticular dermis is Type I, it is assumed in the following derivation that the resistance to flow is from Type I collagen fibrils ( $\sim 80 \mathrm{~nm}$ diameter).

It is assumed that drag due to individual cells is negligible, and while the interstitium has a fixed negative charge, it is assumed that electrokinetic effects are negligible compared to hydraulic resistance $[54,55]$. Hydraulic conductivity $\kappa\left[\mathrm{m}^{4} \cdot \mathrm{N}^{-1} \cdot \mathrm{s}^{-1}\right]$ is defined as:

$$
\kappa=\frac{k}{\mu},
$$

and hydraulic resistivity (or drag) $R^{\prime}\left[\mathrm{N} \cdot \mathrm{s} \cdot \mathrm{m}^{-4}\right]$ is:

$$
R^{\prime}=\frac{1}{\kappa}
$$

where $\mu$ is the dynamic viscosity of the fluid $\left(1 \mathrm{mPa} \cdot \mathrm{s}\right.$ for water at $\left.20^{\circ} \mathrm{C}\right)$. Hydraulic conductivity refers to the flow per unit pressure drop across a unit area of material of unit thickness. In this derivation, specific hydraulic resistivity $R\left[\mathrm{~m}^{-2}\right]$ is the reciprocal of specific hydraulic conductivity, $k$, or permeability. Permeability, the conductivity to a fluid of unit viscosity, is purely a material property.

The Carman-Kozeny equation describes the factors affecting $k[56]$,

$$
k=\frac{\epsilon^{3}}{G S^{2}}=\frac{\epsilon \bar{r}^{2}}{G}
$$

where $G$ is the Kozeny factor (dependent on channel shape and tortuosity), $S$ is the wetted surface area, and $\bar{r}(=\epsilon / S)$ is the mean hydraulic radius of the flow channels. $G$ is dependent on $\epsilon$, and can be calculated using Happel and Brenner's relation for beds of neutral uniform cylindrical fibers [57]. For cylinders aligned parallel to flow,

$$
G_{p}=\frac{2 \epsilon^{3}}{\phi\left[2 \ln (1 / \phi)-3+4 \phi-\phi^{2}\right]}
$$

where $\phi=1-\epsilon$ is the fractional solid volume. For cylinders aligned perpendicular to 
flow,

$$
G_{r}=\frac{2 \epsilon^{3}}{\phi\left[\ln \left(\frac{1}{\phi}\right)-\left(\frac{1-\phi^{2}}{1+\phi^{2}}\right)\right]} .
$$

$G$ can then be calculated, assuming that the interstitium consists of uniform cylinders randomly oriented in three planes:

$$
G=\frac{2}{3} G_{r}+\frac{1}{3} G_{p}
$$

To determine the effect of collagen fibrils alone, the fractional solid volume ( $\phi=$ $1-\epsilon)$ of the fibrils must be calculated. Since collagen fibrils contain both water and collagen, the effective specific volume $\left(v_{c}^{\prime}\right)$, adjusted for water content must be considered. The fractional solid volume occupied by collagen fibrils $\left(\phi_{c}\right)$ is then,

$$
\phi_{c}=C_{c} v_{c}^{\prime}
$$

where $C_{c}$ is the collagen concentration. Assuming that the fibrils have a cylindrical geometry with mean effective fiber radius $r$, the fiber surface area can be calculated by,

$$
S=2 \pi r l=\frac{2 \phi}{r} .
$$

Substituting Equations 5.9 and 5.10 into Equation 5.5 gives the specific hydraulic resistivity due to collagen fibrils:

$$
R_{\text {collagen }}=\frac{1}{k_{\text {collagen }}}=\frac{4 G\left(C_{c} v_{c}^{\prime}\right)^{2}}{\left(1-C_{c} v_{c}^{\prime}\right)^{3} r^{2}}
$$

The values used to calculate $R_{\text {collagen }}$ are given in Table 5.1.2. The median values of $r_{c}=40 \mathrm{~nm}$ and $C_{c}=0.35 \mathrm{mg} / \mathrm{mm}^{3}$ were used $\left(C_{c}\right.$ is reported to be between 0.3 and $\left.0.4 \mathrm{mg} / \mathrm{mm}^{3}[58,59,60,61]\right) . R_{\text {collagen }}$ was calculated to be $1.31 \times 10^{17} \mathrm{~m}^{-2}$.

GAG and PGP have a low volume fraction when compared to collagen fibrils, but their contribution to flow resistance is significant. Their wide range of radii makes the derivation of hydraulic resistance less direct.

The solid volume fraction of GAG and PGP in the extra fibrillar space $\left(\phi_{E F}\right)$ can 
Table 5.1: Values used to calculate $R_{\text {collagen }}$

\begin{tabular}{|l|c|l|}
\hline Symbol & Value & Source \\
\hline \hline$\phi_{C}$ & 0.661 & {$[51,52]$} \\
$\epsilon_{C}$ & 0.339 & $\epsilon=1-\phi$ \\
$G_{p}$ & 3.36 & Equation 5.6 \\
$G_{r}$ & 5.325 & Equation 5.7 \\
$G$ & 4.67 & Equation 5.8 \\
$v_{c}^{\prime}$ & $1.89 \mathrm{~mm}^{3} / \mathrm{g}$ & Equation 5.9 \\
$r_{c}$ & $40 \mathrm{~nm}$ & {$[3]$} \\
$C_{c}$ & $350 \mathrm{mg} / \mathrm{cm}^{3}$ & {$[58,59,60,61]$} \\
\hline
\end{tabular}

be calculated as follows:

$$
\phi_{E F}=\frac{\phi_{G}+\phi_{P}}{1-\phi_{c}}
$$

where $\phi_{G}$ and $\phi_{P}$ are the solid volume fractions of GAG and PGP, respectively.

The hydraulic conductivity of a matrix containing two fiber species with different radii is given by the relation derived by Ethier [62]:

$$
\kappa_{P G}=\left(\frac{l_{P}}{\kappa_{P}}+\frac{l_{G}}{\kappa_{G}}\right)^{-1}
$$

where $\kappa_{P G}$ is the hydraulic conductivity due to GAG and PGP, $l_{P}$ and $l_{G}$ are the fractional lengths of PGP and GAG per unit volume, and $\kappa_{G}$ and $\kappa_{P}$ are the hydraulic conductivities of GAG and PGP, respectively. Since by definition $l_{1}=L_{1} /\left(L_{1}+L_{2}\right)$, where $L$ is the total fiber length, and $L=\phi /\left(\pi r^{2}\right)$,

$$
l_{G}=\frac{\frac{\phi_{G}}{r_{G}^{2}}}{\frac{\phi_{G}}{r_{G}^{2}}+\frac{\phi_{P}}{r_{P}^{2}}},
$$

and

$$
l_{P}=1-l_{G}
$$

$\kappa_{G}$ was experimentally determined by Preston et al. [63] to be:

$$
\kappa_{G}\left[\mathrm{~m}^{4} /(\mathrm{N} \cdot \mathrm{s})\right]=1.91 \times 10^{-17} \cdot\left(C_{G}\right)^{-1.485}
$$


where $C_{G}$ is the concentration of GAG (in $\mathrm{mg} / \mathrm{mm}^{3}$ ). $\quad C_{G}$ is $0.0034 \mathrm{mg} / \mathrm{mm}^{3}$ $[58,59,60,61]$ and thus $\kappa_{G}$ is $8.85 \times 10^{-14} \mathrm{~m}^{4} /(\mathrm{N} \cdot \mathrm{s}) . \kappa_{P}$ can be calculated from Equation 5.5, 5.9 and 5.10:

$$
\kappa_{P}=\frac{\left(1-\phi_{P}\right)^{3} r_{P}^{2}}{4 \cdot \mu \cdot G \cdot \phi_{P}^{2}}
$$

Table 5.2: Values used to calculate $\kappa_{P}$

\begin{tabular}{|l|c|l|}
\hline Symbol & Value & Source \\
\hline \hline$C_{p}$ & $0.35 \mathrm{mg} / \mathrm{mm}^{3}$ & {$[58,59,60,61]$} \\
$v_{p}^{\prime}$ & $1000 \mathrm{~mm}^{3} / \mathrm{g}$ & {$[51]$} \\
$\phi_{P}$ & 0.0085 & Equation 5.9 \\
$\epsilon_{P}$ & 0.9915 & $\epsilon=1-\phi$ \\
$G_{p}$ & 34.9 & Equation 5.6 \\
$G_{r}$ & 60.9 & Equation 5.7 \\
$G$ & 52.2 & Equation 5.8 \\
$r$ & $1 \mathrm{~nm}$ & {$[51]$} \\
\hline
\end{tabular}

The values used in the calculation of $\kappa_{P}$ are shown in Table 5.1.2. $v_{p}^{\prime}$ was estimated from the usual partial specific volume, $\bar{v}$, for protein $(\sim 0.7)$. The effective specific volumes of hydrophilic proteins are generally $\sim 30 \%$ greater than $\bar{v} . \kappa_{P}$ was calculated to be $6.46 \times 10^{-14} \mathrm{~m}^{4} /(\mathrm{N} \cdot \mathrm{s})$.

Using Equations 5.14 and $5.15, l_{G}$ was calculated to be 0.538 and $l_{P}$ was calculated to be $0.462 . \kappa_{P G}$ was calculated from Equation 5.13 to be $7.38 \times 10^{-14} \mathrm{~m}^{4} /(\mathrm{N} \cdot \mathrm{s})$.

Factoring in tortuosity $(\zeta)$ and accounting for the space occupied by the collagen fibrils gives the specific hydraulic resistivity due to GAG and PGP $\left(R_{P G}\right)$ :

$$
R_{P G}=\frac{1}{\mu \cdot \kappa_{P G} \cdot \zeta\left(1-\phi_{\text {collagen }}\right)} .
$$

$\zeta$ was estimated to have a value of 0.7 [64]. Thus, $R_{P G}$, was calculated to be 5.7 $\times 10^{16} \mathrm{~m}^{-2}$. It is important to note that even though $\phi_{G}$ and $\phi_{P}$ are much lower than $\phi_{C}$, the drag due GAG and PGP is not insignificant when compared to the drag due to collagen $\left(R_{\text {collagen }}=1.31 \times 10^{17} \mathrm{~m}^{-2}\right)$.

The total hydraulic resistance $\left(R_{I S}\right)$ due to collagen fibrils, GAG, and PGP is 
therefore:

$$
R_{I S}=R_{\text {collagen }}+R_{P G}
$$

The total hydraulic resistance $\left(R_{I S}\right)$ was calculated to be $1.87 \times 10^{17} \mathrm{~m}^{-2}$, corresponding to a permeability, $k_{I S}$, of $5.36 \times 10^{-18} \mathrm{~m}^{2}$. As a reference point, the permeability across a capillary wall is $10^{-17} \mathrm{~m}^{-2}$ [65]. For comparison, the permeability of rat dermis has been experimentally determined (ex vivo) to be $2-6 \times 10^{-18}$ $\mathrm{m}^{2}[66]$.

\subsubsection{Change in Tissue Permeability due to Swelling}

During the injection process, the tissue swells near the injection site, resulting in an increase in the local porosity. The derivation in Section 5.1.2 assumes that the porosity is uniform and remains constant, which is clearly not the case during an injection. It is thus important to consider the effect of tissue swelling on tissue permeability in the interstitium. Tissue permeability is dependent on $\phi_{c}, \phi_{P G P}$, and $C_{G}$ which all change with tissue swelling.

The calculated permeabilities for different porosity values are shown in Figure 5-1. The permeability can change by several orders of magnitude for even a small change in porosity. The permeability approaches zero as the porosity decreases since the material behaves more like a solid, while the permeability approaches infinity as the porosity increases since the porous media behaves more like an open material.

The relationship between volumetric strain, which describes the tissue swelling, and $\phi_{c}$ and $\phi_{P G P}$ can be obtained by considering a single pore 'element.'

Consider a single, box-like pore 'element,' where the collagen fibrils comprise the edges of the box (Figure 5-2). The volumetric strain, $e$, is the sum of the strains in the principal directions:

$$
e=\epsilon_{x}+\epsilon_{y}+\epsilon_{z}=\frac{\delta x}{x_{0}}+\frac{\delta y}{y_{0}}+\frac{\delta z}{z_{0}} \approx \frac{\Delta V}{V_{0}}
$$

where $\Delta V$ is the change in volume and $V_{0}$ is the original volume of the element. 


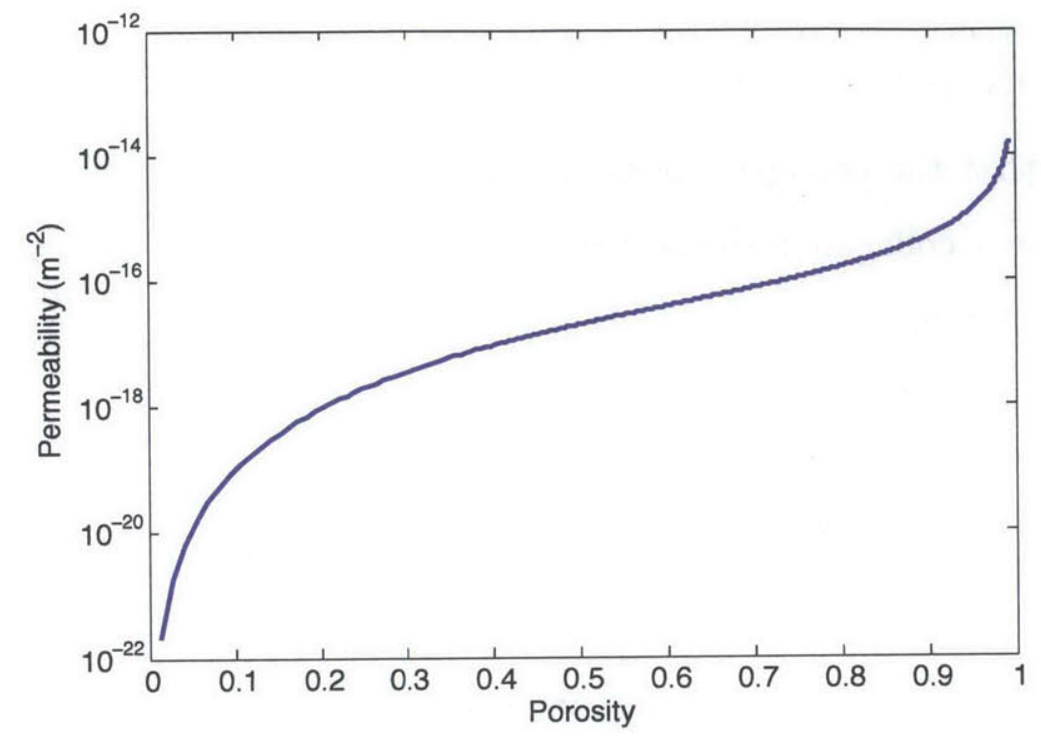

Figure 5-1: The permeability changes by several orders of magnitude with a change in porosity.

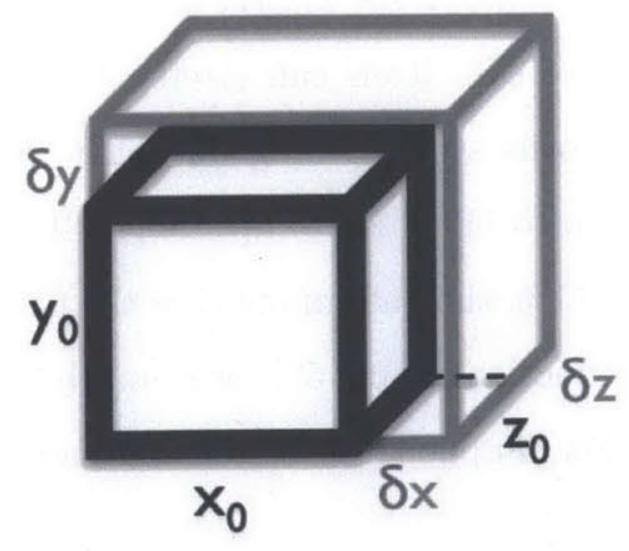

Figure 5-2: Box-like pore 'element' used to model the effect of swelling on dermis permeability. 
The total volume of the element is: $V=V_{0}+\Delta V$. Substituting in Equation 5.21 gives:

$$
V=V_{0}(1+e)
$$

Assuming that the collagen fibrils are incompressible $(\nu=0.5)$, we can assume that the volume of collagen in the pore element remains constant. Thus, the fractional volume of collagen in swelled tissue, $\phi_{C, s}$, is:

$$
\phi_{C, s}=\frac{\text { Collagen volume }}{\text { Total Volume, } V}=\frac{\text { Collagen volume }}{V_{0}(1+e)}=\phi_{C, 0}\left(\frac{1}{1+e}\right) \text {, }
$$

where $\phi_{C, 0}$ is the fractional volume of collagen for unswelled tissue.

Substituting Equation 5.22 into Equation 5.11 and simplifying gives the resistance to interstitial flow due to collagen in swelled tissue, $R_{\text {collagen,s }}$ :

$$
R_{\text {collagen }, s}=\frac{4 G\left(\phi_{c, s}\right) \phi_{c, 0}^{2}(1+e)}{r_{c}^{2}\left(\epsilon_{c, 0}+e\right)^{3}}
$$

where $\epsilon_{c, 0}$ is the porosity of the unswelled tissue. Note that the Kozeny factor, $G$, is also a function of $\phi_{C, s}$. Equation 5.24 assumes that the radius of the fibrils remain relatively unchanged. In a collagenous matrix such as skin, the collagen fibrils are randomly coiled. While the skin itself can stretch up to $115 \%$ [67], much of this stretching is due to the fibrils straightening out. The fibrils themselves can only undergo an elongation of up to $11 \%$ before rupture [4]. If we consider that the length of a fibril only changes by $11 \%$, then assuming that the fibers are incompressible, the radius should undergo a reduction of only $5 \%$, or $2 \mathrm{~nm}$, thus validating the assumption of constant fibril radius. For simplicity, it is assumed there there is no rupture of the collagen fibers.

A similar argument can be made for the contribution of proteoglycans to interstitial flow resistance. Again, we have:

$$
\phi_{P G P, s}=\frac{\text { PGP volume }}{\text { Total Volume, } V}=\frac{\text { PGP volume }}{V_{0}(1+e)}=\phi_{P G P, 0}\left(\frac{1}{1+e}\right)
$$


where $\phi_{P G P, s}$ is the fractional volume of proteoglycans in swelled tissue and $\phi_{P G P, 0}$ is the fractional volume of proteoglycans in unswelled tissue.

Since the resistance to interstitial flow due to GAGs is concentration-dependent, and the mass of GAGs in the interstitium remains constant, we have:

$$
C_{G, s}=\frac{\operatorname{mass}_{G}}{\text { Vol }_{\text {tot }}}=\frac{\operatorname{mass}_{G}}{V_{0}(1+e)}=C_{G 0}\left(\frac{1}{1+e}\right)
$$

where $C_{G, s}$ is the concentration of GAGs in the swelled tissue, and $C_{G 0}$ is the concentration of GAGs in the unswelled tissue (units $=\left[\mathrm{kg} / \mathrm{m}^{3}\right]$ ). Then, the contribution of GAGs to dermal permeability in swelled tissue is:

$$
k_{G, s}=1.91 \times 10^{-20} \cdot\left(C_{G}\right)^{-1.485}=1.91 \times 10^{-20} \cdot\left(\frac{C_{G 0}}{1+e}\right)^{-1.485}
$$

The manipulations described in Section 5.1.2 to factor in tortuosity, adjust for the space occupied by the collagen fibrils, factor in the different radii of PGP and GAG, and combine $k_{P G P, s}, k_{G A G, s}$, and $k_{\text {collagen,s }}$ can then be performed to get:

$$
k_{I S}=\left[\frac{4 G\left(\phi_{c}\right) \phi_{c, 0}^{2}(1+e)}{r_{c, 0}^{2}\left(\epsilon_{c, 0}+e\right)^{3}}+\frac{\left[\frac{0.538}{1.91 \times 10^{-20}\left(\frac{C_{G, 0}}{1+e}\right)^{-1.485}}+\frac{1.848 \cdot G\left(\phi_{P G P}\right) \phi_{P G P, 0}^{2}(1+e)}{r_{P G P, 0}^{2}\left(\epsilon_{P G P, 0}+e\right)^{3}}\right]}{\zeta\left[1-\phi_{c, 0}\left(\frac{1}{1+e}\right)\right]}\right]^{-1}
$$

Equation 5.27 was used to calculate the dermal permeability, $k_{I S}$, as a function of volumetric strain (Figure 5-3). As expected, the permeability increases by several factors as the volumetric strain is increased and the tissue swells. The permeability also decreases nearly to zero when the volumetric strain is negative and the tissue is compressed. As the tissue is compressed, the tissue no longer resembles a porous matrix and behaves more like a solid. 


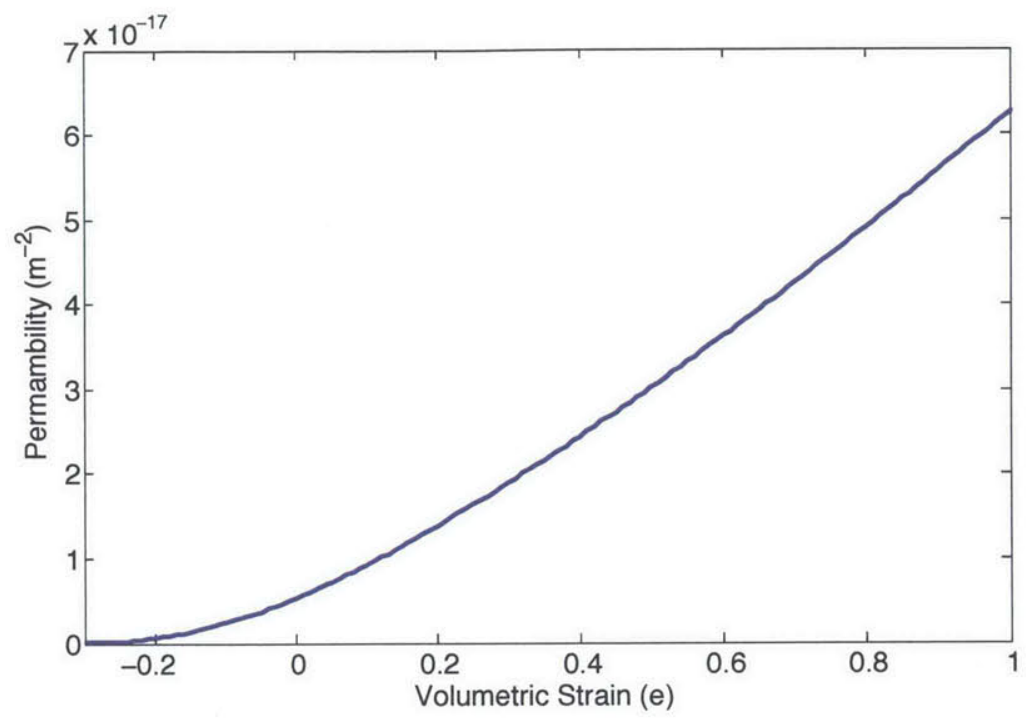

Figure 5-3: Calculated dermal permeability, $k_{I S}$, as a function of volumetric strain.

\subsubsection{Dermis model}

The dermis was modeled in Comsol Multiphysics 4.3a using the Poroelasticity module, which couples porous media flow with elastic deformations of the media. It is assumed that the drained matrix is a porous matrix of collagen with permeability defined by Equation 5.27 and water as the fluid.

The Poroelasticity module requires the user to define the structural material properties of the porous matrix, such as the Young's modulus, E, and the shear modulus, G. As described in Section 3.1.1, the mechanical behavior of the dermis is nonlinear (' $\mathrm{J}$ '-shaped). For simplicity, it is assumed that the drained collagen matrix is a linear, elastic, isotropic material.

\section{Solute Transport}

The dominating mechanism for solute transport for ISF extraction is convection, and diffusion can be ignored. This assumption is validated by considering the Peclet number, $P e$, which compares the convective rate to the diffusive transport rate:

$$
P e=\frac{l_{\text {char }} \cdot v}{D}
$$


where $l_{\text {char }}$ is the characteristic length, $v$ is the fluid velocity, and $D$ is the diffusion coefficient. The value of $l_{\text {char }}$ is on the order of $1 \mathrm{~mm}$ (the thickness of the dermis), $v$ is approximately $1 \mathrm{~mm} / \mathrm{s}$, and $D$ is on the order of $10^{-10} \mathrm{~m}^{2} / \mathrm{s}$ for most solutes in water. This gives $P e=10^{4}$, and diffusion can be ignored.

The poroelasticity module was coupled with the solute transport module to model the behavior of solutes contained in the interstitial fluid. There are several different phenomena which contribute to the mass transport of solutes in a porous medium. The collagen fibers act as obstacles to fluid flow (Figure 5-4). When fluid flows through pores, there is additional mixing within the pores due to local variations in pore fluid velocities. This mixing is known as dispersion, and is an important parameter in the consideration of subsurface fluid flow. Additionally, the spreading in the direction of fluid flow (longitudinal dispersion, $\alpha_{l}$ ) is not equal to spreading perpendicular to fluid flow (transverse dispersion, $\alpha_{t r}$ ). The longitudinal dispersion tends to be about an order of magnitude higher than the transverse dispersion.

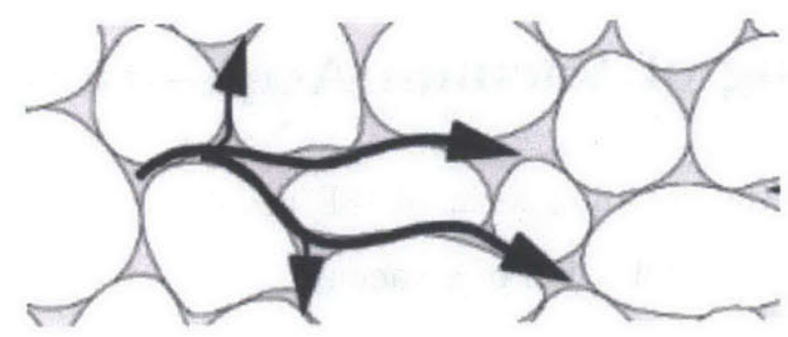

Figure 5-4: When fluid flows through porous media, the fluid must flow around solid particles, resulting in local variations in pore fluid velocities and additional mixing.

To determine the theoretical values of dispersivity in the dermis, it is useful to look at an analogous topic which has been more thoroughly researched: mass transport in soils. Diffusion and dispersion in porous rocks is of interest to the oil industry, and a great deal of research on fluid flow through porous beds has already been done. Raimondi et al. showed that $\alpha_{l}$ can be given by the following expression:

$$
\alpha_{l}=c \sigma d_{p}
$$

where $c$ is a constant, $\sigma$ is a measure of the inhomogeneity of the soil pack, and $d_{p}$ 
is the diameter of the average particle [68]. A number of researchers have shown that the mixing-cell theory leads to a value of $c=0.5[69,70,71]$. The value of $\sigma$ is usually determined experimentally, but has smaller values for random packs of large beads and larger values for poorly packed beads and smaller beads (larger beads pack better than smaller beads) [72]. A perfectly packed bed of uniform beads will have the theoretical minimal values of $\sigma=1$.

Tranverse dispersivity, $\alpha_{t r}$, will have a value that is approximately an order of magnitude smaller than $\alpha_{l}$. Perkins et al. stated that $\alpha_{t r}$ can be found by the following expression [72]:

$$
\alpha_{t r}=0.0157 \sigma d_{p}
$$

The results from Section 3.4 were used to estimate $d_{p}$, and $\sigma$ was estimated from the literature. $\alpha_{l}$ was modeled to be approximately $0.001 \mathrm{~m}$ and $\alpha_{t r}$ was approximately $0.00003 \mathrm{~m}$.

\subsection{Modeling of Vacuum Acquisition Methods}

This section models the common form of ISF acquisition: removing the epidermis and collecting ISF with application of a vacuum.

\subsubsection{Model setup}

The poroelasticity module along with the transport of chemical species module were used. A time-dependent study, which calculated the deformation of the dermis, velocity field of the fluid, and the velocity and transport of the mass species was applied.

The geometry and boundary conditions used for the finite element analysis is shown in Figure 5-5. An axisymmetric geometry with side $e$ as the centerline was used. The boundary conditions for the solid mechanics interface, the solute transport module, and the fluid dynamics interface are also shown. It is assumed that the stratum corneum is removed to form a hole (side $a$ ) with a radius of $r_{\text {hole }}=0.25$ $\mathrm{mm}$ through which the fluid is acquired. The orifice was set to be immobile (fixed) 
and fluid was allowed to flow through this boundary (open boundary). A pressure profile was set on this boundary to simulate the application of a vacuum. Side $b$ is the surface of the skin, and was set to be immobile (fixed) and impermeable (no solute flux and no fluid flow). The tissue is assumed to be an infinite size such that there are no edge effects from the boundary conditions at sides $c$ and $d$. Sides $c$ and $d$ are therefore arbitrary boundaries, and the conditions on either side of sides $c$ and $d$ are equal. Sides $c$ and $d$ are free to move, and both solutes and fluid are free to flow through the boundary.

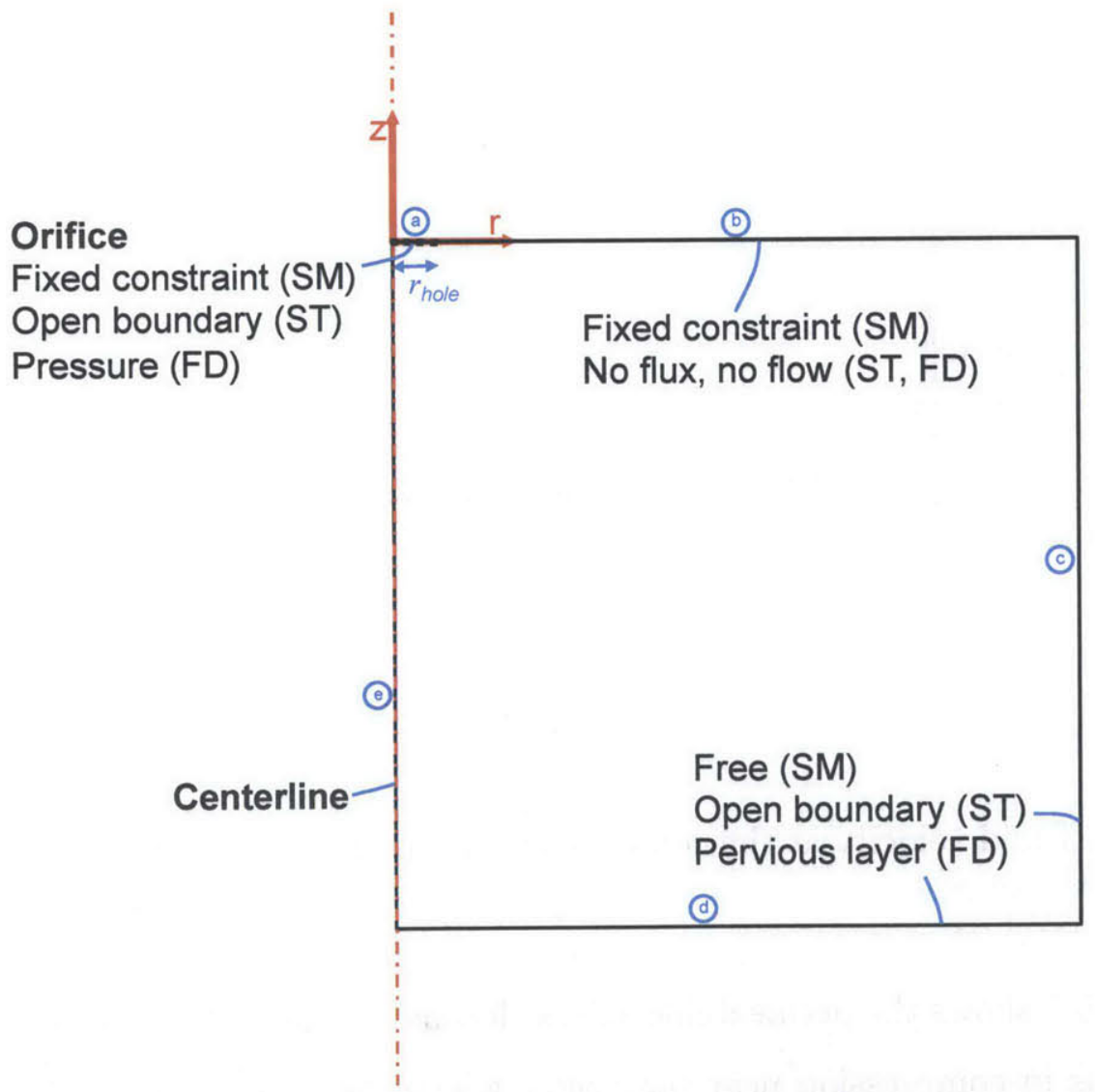

Figure 5-5: The geometry and boundary conditions used to simulate a vacuum-only acquisition. An axisymmetric geometry with side $e$ as the centerline was used. SM stands for the solid mechanics interface, ST stands for the solute transport module, and FD stands for the fluid dynamics interface. 


\subsubsection{Results}

The magnitude of the velocity of the fluid flowing out of the center of the orifice is shown in Figure 5-6. The velocity has a peak value of $1.5 \mu \mathrm{m} / \mathrm{s}$ when the vacuum is first applied, but the velocity quickly drops to a steady-state value of $0.45 \mu \mathrm{m} / \mathrm{s}$. Since the velocity of the fluid is on the order of $1 \mu \mathrm{m} / \mathrm{s}$, it is clear why such collection methods must apply vacuums for tens of minutes. According to the simulation, the total volume of fluid collected through the orifice in 5 minutes was $0.20 \mu \mathrm{L}$. To collect more fluid, one must either apply the vacuum over a larger area, or apply the vacuum for a longer period of time.

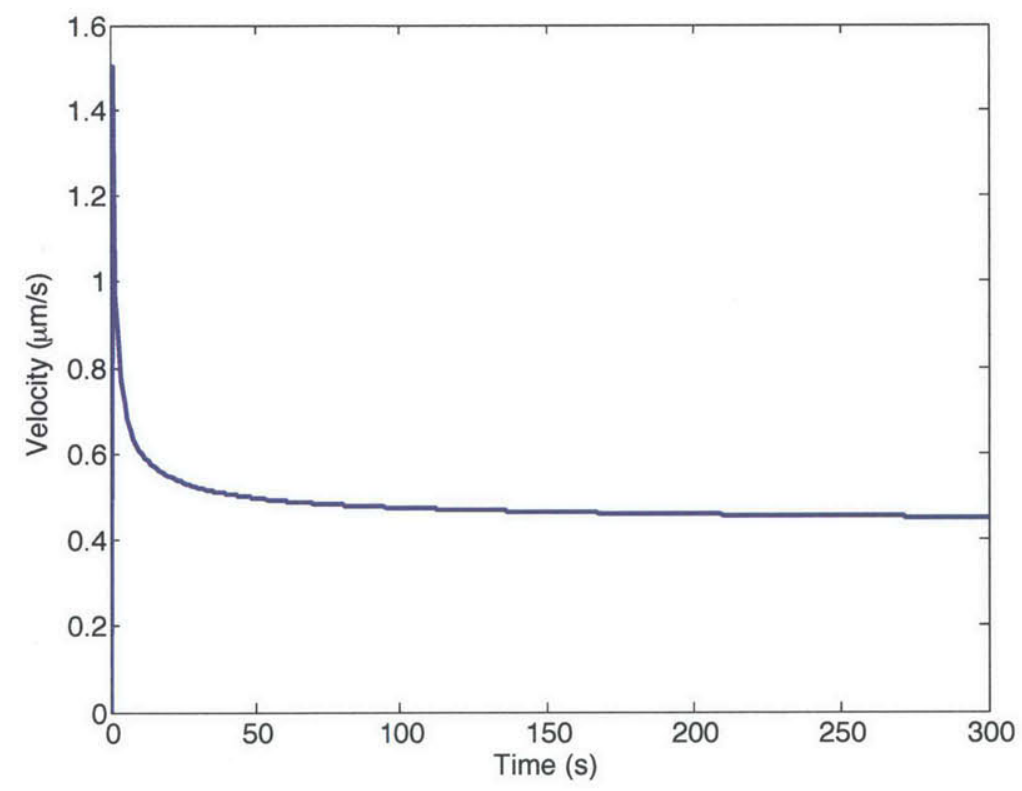

Figure 5-6: Fluid velocity at the center of the orifice when a vacuum of $-66.1 \mathrm{kPa}$ was applied at the orifice.

Figure 5-7 shows the permeability of the dermis at time $t=3 \mathrm{~s}$. Note that because the tissue is in compression near the orifice where the vacuum is applied, there is a decrease in permeability. This further impedes the fluid flow out of the orifice.

Figure 5-8 shows the pressure distribution in the tissue (represented by colors) as well as the fluid flow within the tissue. The fluid flow within the tissue is represented by the arrows: the velocity magnitude is represented by the size of the arrow and the direction of fluid flow is depicted by the direction the arrow is pointing. It is 


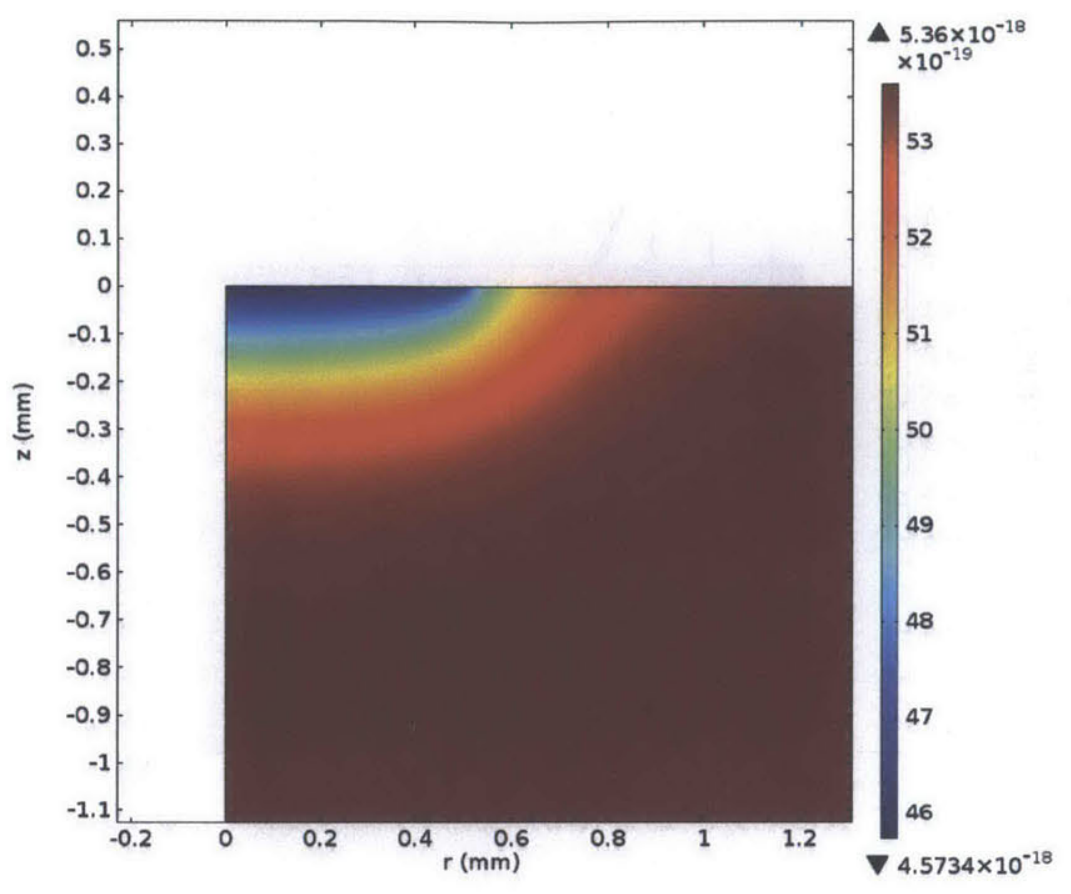

Figure 5-7: Tissue permeability during a simple vacuum acquisition method. Color bar represents tissue permeability in $\left[\mathrm{m}^{2}\right]$.

also important to note that most of the fluid flow is occurring at depths down to $300 \mu \mathrm{m}$ below the surface of the skin, indicating that most of the analytes will be collected from a small localized area just below the skin surface.

\subsection{Modeling of JI ISF Acquisition Method}

To utilize the jet injector for interstitial fluid acquisition, the skin must first be breached. This is achieved by injecting first with a set amount of fluid. The injectate is allowed to mix with the interstitial fluid in the tissue, and then a vacuum is created in the ampoule to collect the injectate and ISF mixture. This section explores that acquisition strategy and models the effect of different injection and extraction parameters on the acquisition process. 


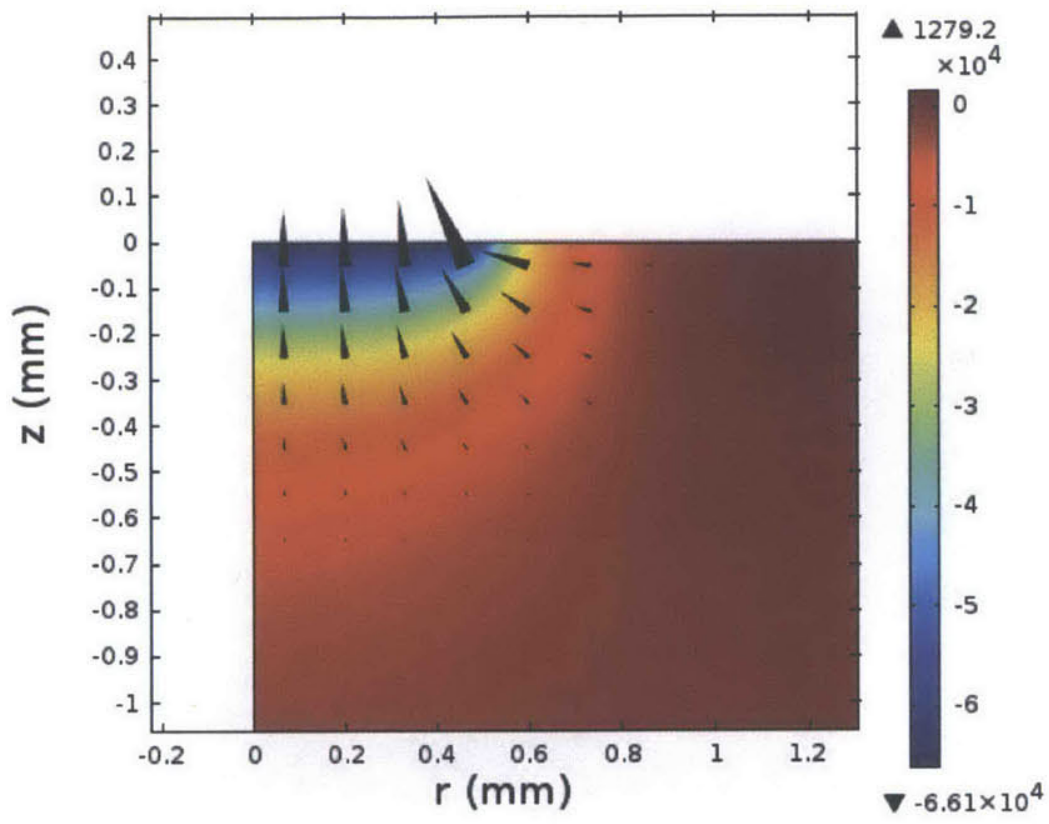

Figure 5-8: Pressure distribution in the tissue during a vacuum acquisition (color bar represents pressure in $[\mathrm{Pa}]$ ). The arrows depict fluid velocities (direction and magnitude).

\subsubsection{Modeling Injection}

Jet injection is a complex process that involves turbulent fluid dynamics, tissue tearing, and fracture flow. However, finite element modeling packages are, at this point in time, limited in their abilities to model the fracture mechanics of a nonlinear, anisotropic material such as biological tissue while also considering the turbulent fluid behavior that one sees during jet injections. A much simplified model of the injection process was created in Comsol, which modeled a slow (laminar flow) injection, with the penetration hole already formed (did not account for fracture mechanics).

\section{Geometry and Boundary Conditions}

The geometry and boundary conditions used to simulate the injection prior to extraction are shown in Figure 5-9. The boundary conditions are similar to the boundary conditions shown in Figure 5-5, but the geometry is slightly different, as it is assumed that the needle creates a hole in the tissue that has a radius of $r_{\text {hole }}$ and a depth of $l_{\text {hole }}$. For the following simulations, $r_{\text {hole }}=0.25 \mathrm{~mm}$ and $l_{\text {hole }}=1 \mathrm{~mm}$. Similar to 
Figure 5-5, the geometry is axisymmetric with side $f$ as the centerline. The orifice (side $a$ ) was set to be immobile (fixed) and fluid was allowed to flow through this boundary (open boundary). A pressure profile was set on this boundary to simulate the injection and the subsequent application of a vacuum. Side $b$ was immobile and impermeable since it was assumed that the walls of the needle will prevent any further deformation and fluid flow. Side $c$ is the surface of the skin, and was set to be immobile and impermeable. Sides $d$ and $e$ are arbitrary boundaries: free to move, and both solutes and fluid were free to flow through the boundary.

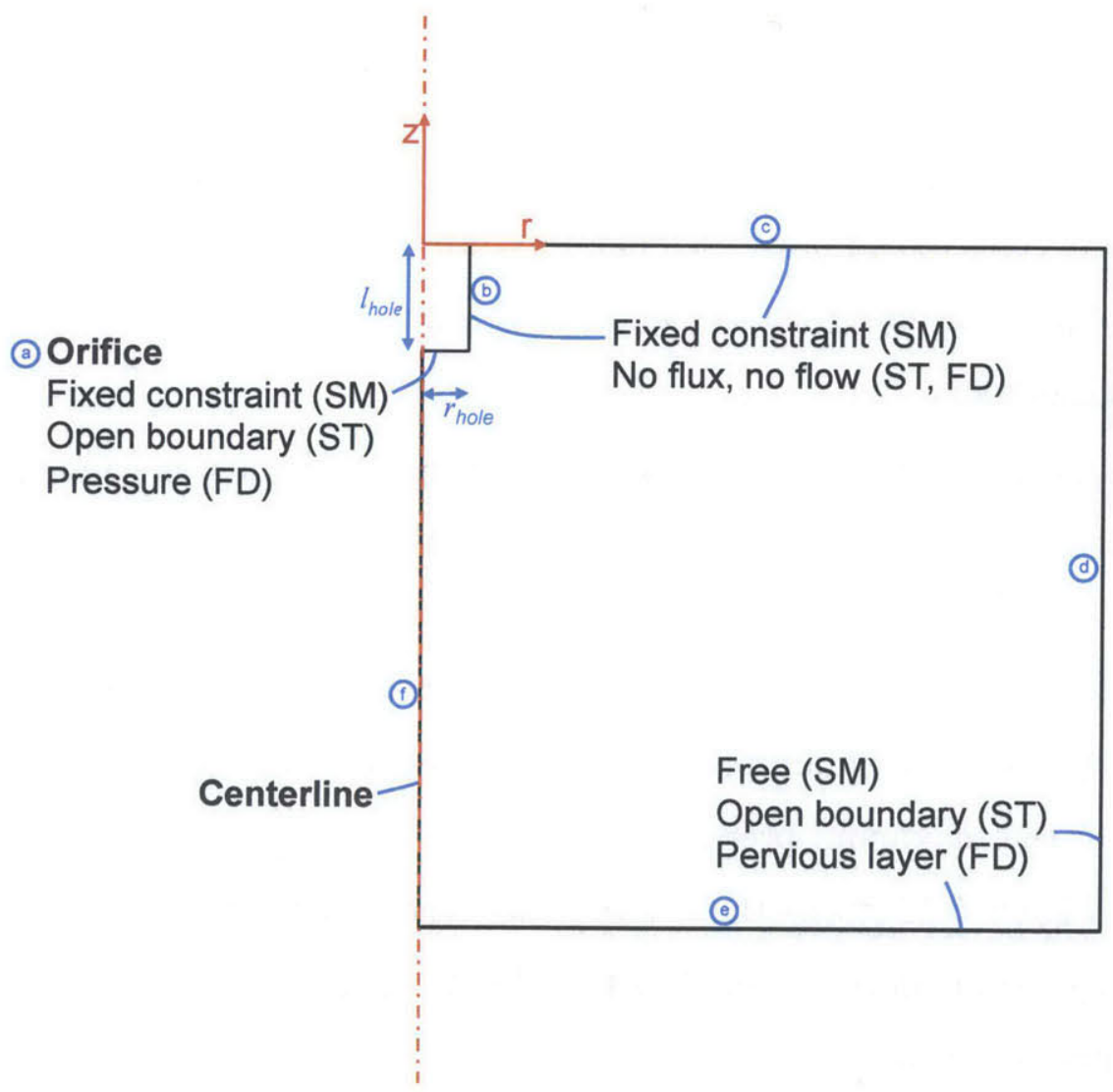

Figure 5-9: The geometry and boundary conditions used to simulate an injection prior to extraction. An axisymmetric geometry with side $f$ as the centerline was used. SM stands for the solid mechanics interface, ST stands for the solute transport module, and FD stands for the fluid dynamics interface. 


\subsubsection{Simulations of the Effect of Injection Parameters}

\section{Effect of Injection Prior to Extraction}

Figure 5-8 shows the pressure distribution of the tissue at the beginning of an extraction, after a volume of $25 \mu \mathrm{L}$ (with a pressure of $29 \mathrm{MPa}$ for $1.8 \mathrm{~s}$ ) had been injected into the tissue. The pressure is represented by the colors and the arrows depict fluid velocities (direction and magnitude). Figure 5-10 shows that injecting the tissue with fluid prior to extraction results in an instantaneous burst of pressure in the tissue (the magnitude of the pressure depends on injection parameters such as injection pressure and volume). This causes a large pressure differential between the injected tissue and the orifice (the pressure difference is on the order of $\mathrm{MPa}$ as opposed to $\mathrm{kPa}$ with no injection), resulting in a greater amount of fluid flow out of the orifice. For this particular set of injection parameters, $2.9 \mu \mathrm{L}$ of total fluid (injectate and ISF mixture) was collected in only $4 \mathrm{~s}$. The mixture contained $0.33 \mu \mathrm{L}$ of ISF, showing that ISF acquisition using the jet injector is a much faster process than other ISF acquisition methods.

The magnitude of the pressure burst caused in the tissue due to injection decays in a few seconds, as the injectate disperses into the surrounding tissue. Figure 5-11 shows the maximum pressure in the tissue as a function of time after injection, for an injection of $25 \mu \mathrm{L}$ of fluid (injection pressure of $29 \mathrm{MPa}$ and injection time of $1.8 \mathrm{~s}$ ). The location of the maximum pressure in the tissue also varies as a function of time; the depth of the maximum pressure increases as the injectate disperses deeper into the tissue. Figure 5-12 shows the depth (below the orifice) of the maximum pressure

in the tissue for an injection of $25 \mu \mathrm{L}$ of fluid (injection pressure of $29 \mathrm{MPa}$ and injection time of $1.8 \mathrm{~s}$ ).

\section{Injection velocity}

The effect of injection velocity on extracted volumes and extracted concentrations for injection volumes of $25 \mu \mathrm{L}$ are shown in Figure 5-13. The injection volume was maintained constant while varying the injection velocity. It should be noted that the 


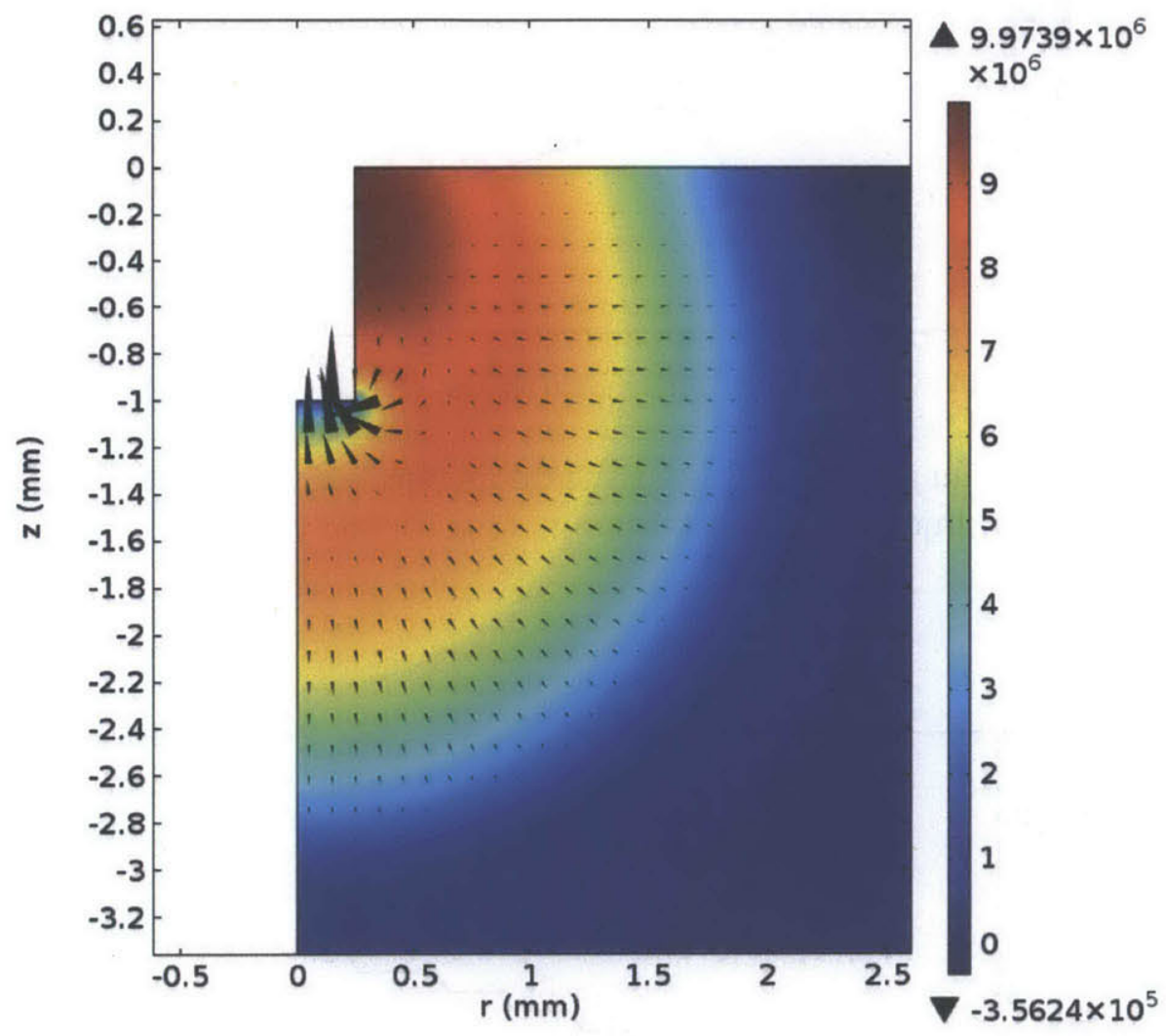

Figure 5-10: Pressure distribution in the tissue during an acquisition, after being injected with $25 \mu \mathrm{L}$ of fluid (color bar represents pressure in $[\mathrm{Pa}]$ ). The arrows depict fluid velocities (direction and magnitude). 


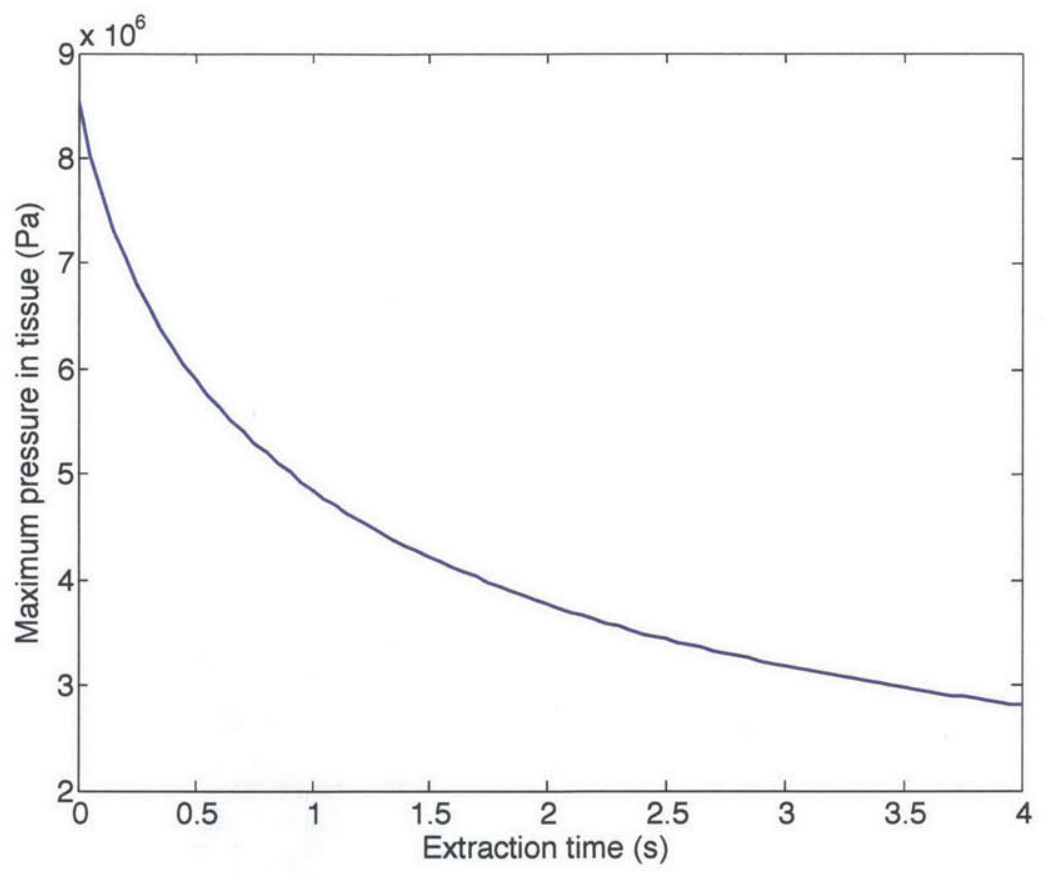

Figure 5-11: Maximum pressure in the tissue for an injected volume of $25 \mu \mathrm{L}$ (injection pressure of $29 \mathrm{MPa}$, injection time of $1.8 \mathrm{~s}$ ).

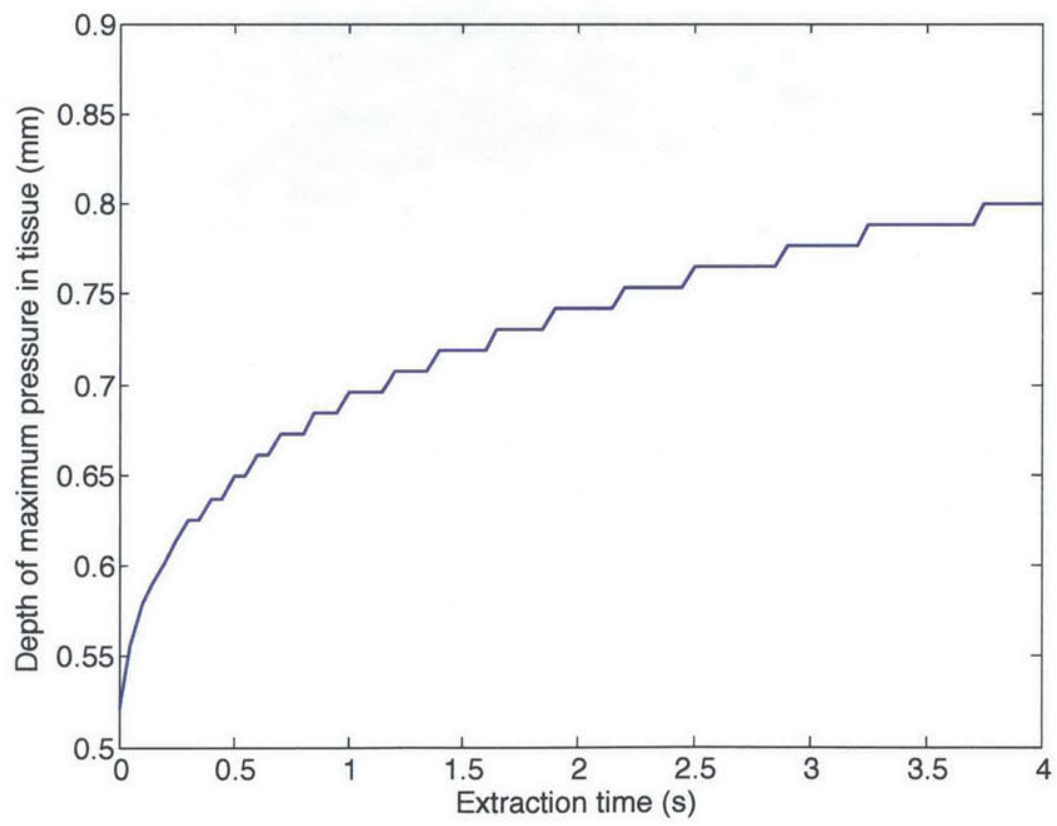

Figure 5-12: Depth of the maximum pressure in the tissue for an injected volume of $25 \mu \mathrm{L}$ (injection pressure of $29 \mathrm{MPa}$, injection time of $1.8 \mathrm{~s}$ ). 
injection velocity is the velocity of the fluid as it enters the tissue, not the jet velocity as it leaves the ampoule orifice (dissimilar from $v_{j e t}$ ). The constant injection volume was achieved by varying the injection pressure and injection time. The same trends were observed for injected volumes of $25 \mu \mathrm{L}$, and $100 \mu \mathrm{L}$ : as the injection velocity is increased, the extracted volume increases slightly. This is because increasing the injection velocity increases the magnitude of the pressure burst in the tissue. Additionally, as the injection velocity increases, the extracted concentration also increases slightly, due to greater mixing from the increased fluid velocity.

\section{Injection Volume}

The length of injection time was varied to investigate the effect of injection volume on the extraction process. The injection pressure $(30 \mathrm{MPa})$ was kept the same across all trials, resulting in the same injection velocity profile for all trials. The results are shown in Figure 5-14 and illustrate that as the injected volume increases, the extracted volume also increases. However, as the injected volume increases, the extracted concentration decreases.

The concentration of the fluid at the center of the orifice as a function of extraction time (or time after injection) is shown in Figure 5-15. As the injection volume is increased, there is greater dilution from the injectate, resulting in lower concentrations of the fluid near the orifice. This results in decreased extracted concentrations.

\subsubsection{Simulations of the Effect of Extraction Parameters}

\section{Pull-back timing}

The length of time between injection and extraction $\left(t_{\text {wait }}\right)$ was varied to investigate the effect of pull-back timing on the extraction process. The injection profile was kept the same across all trials. All trials simulated a $50 \mu \mathrm{L}$ injection with an injection pressure of $35 \mathrm{MPa}$, held for $2.2 \mathrm{~s}$. Wait times of $100 \mathrm{~ms}, 200 \mathrm{~ms}, 300 \mathrm{~ms}, 400 \mathrm{~ms}, 500$ $\mathrm{ms}, 1 \mathrm{~s}$, and $2 \mathrm{~s}$ were simulated. After the set $t_{\text {wait }}$, a vacuum pressure of $-66.1 \mathrm{kPa}$ was applied at the orifice. The resulting extracted volumes and extracted concentrations 


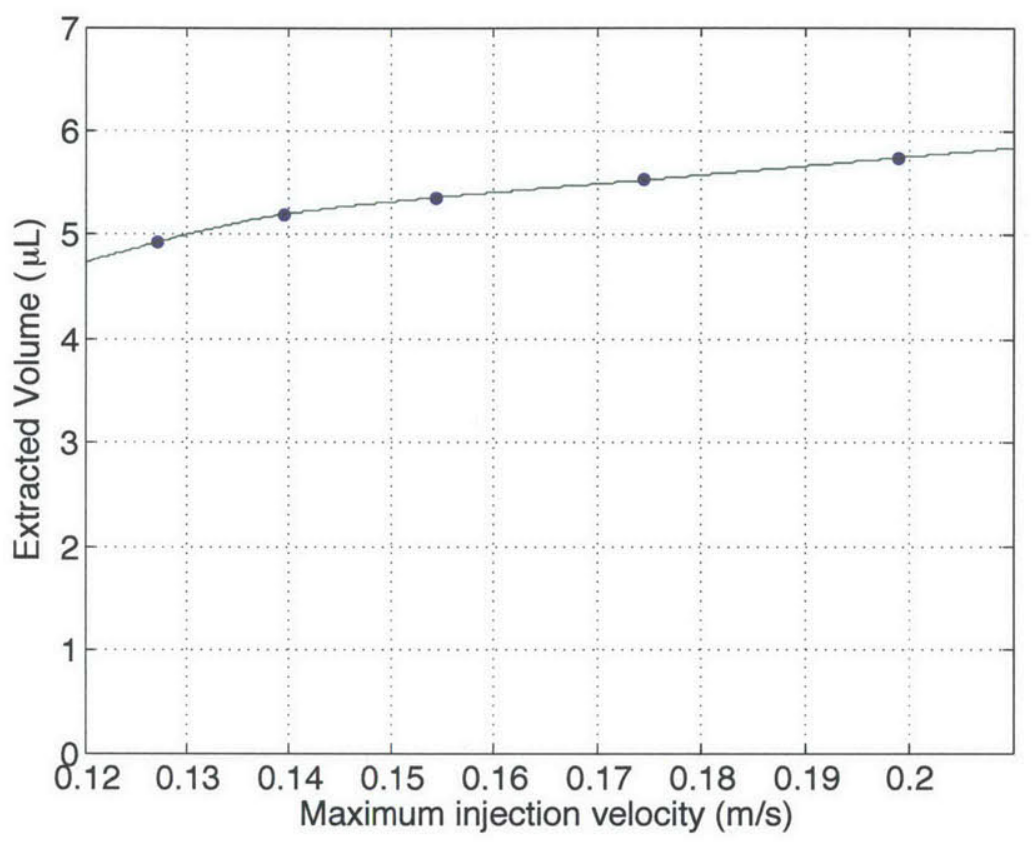

(a) Effect of injection velocity on extracted volume

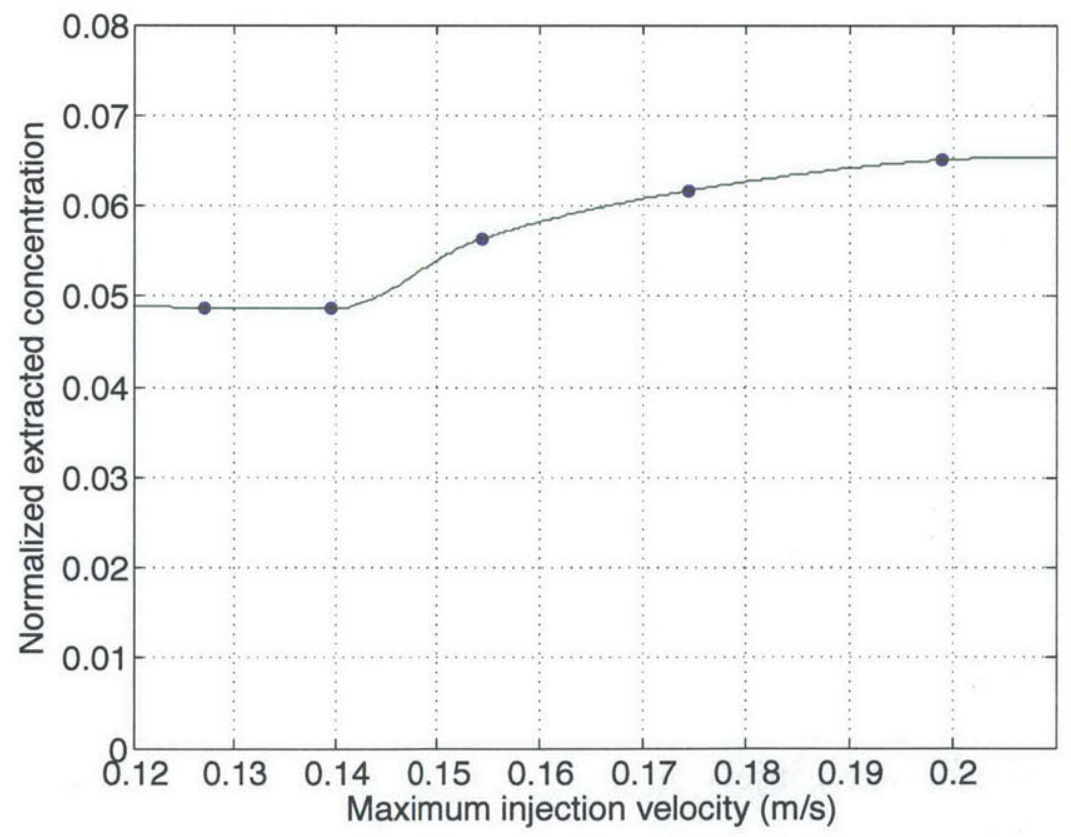

(b) Effect of injection velocity on extracted concentration (normalized)

Figure 5-13: Results showing the effect of injection velocity on extracted volume and concentration, for an injected volume of $50 \mu \mathrm{L}$. 


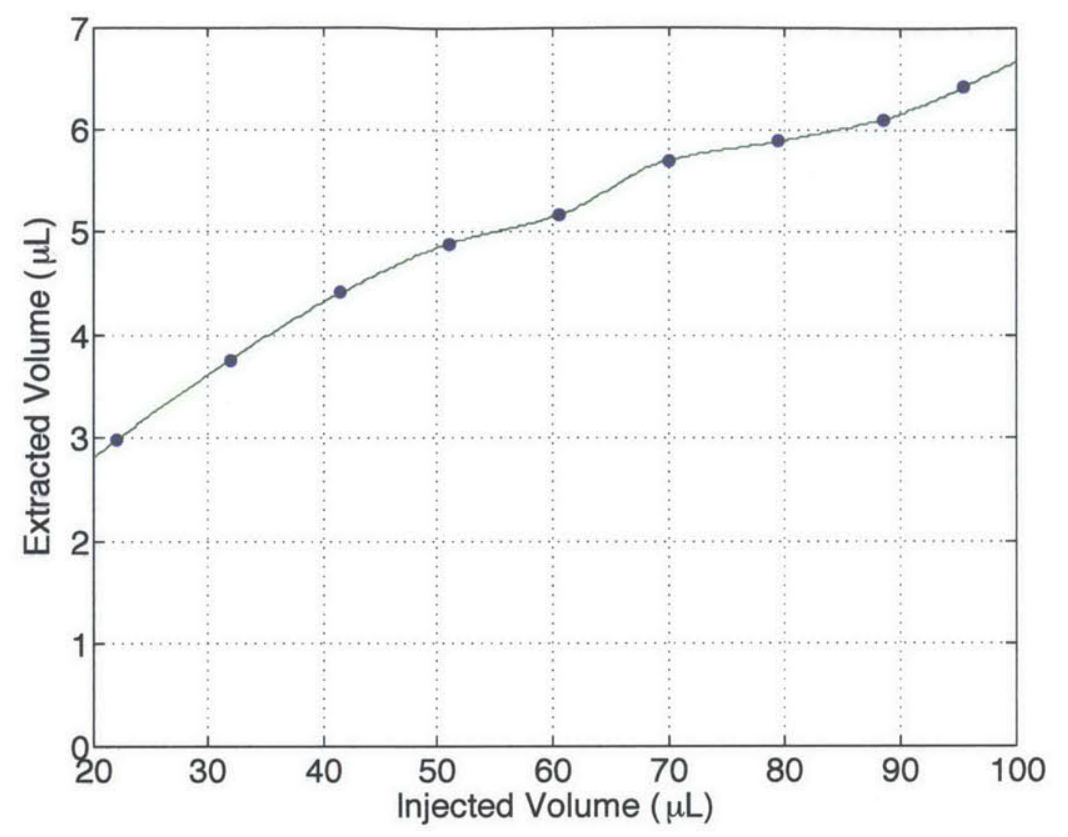

(a) Effect of injected volume on extracted volume

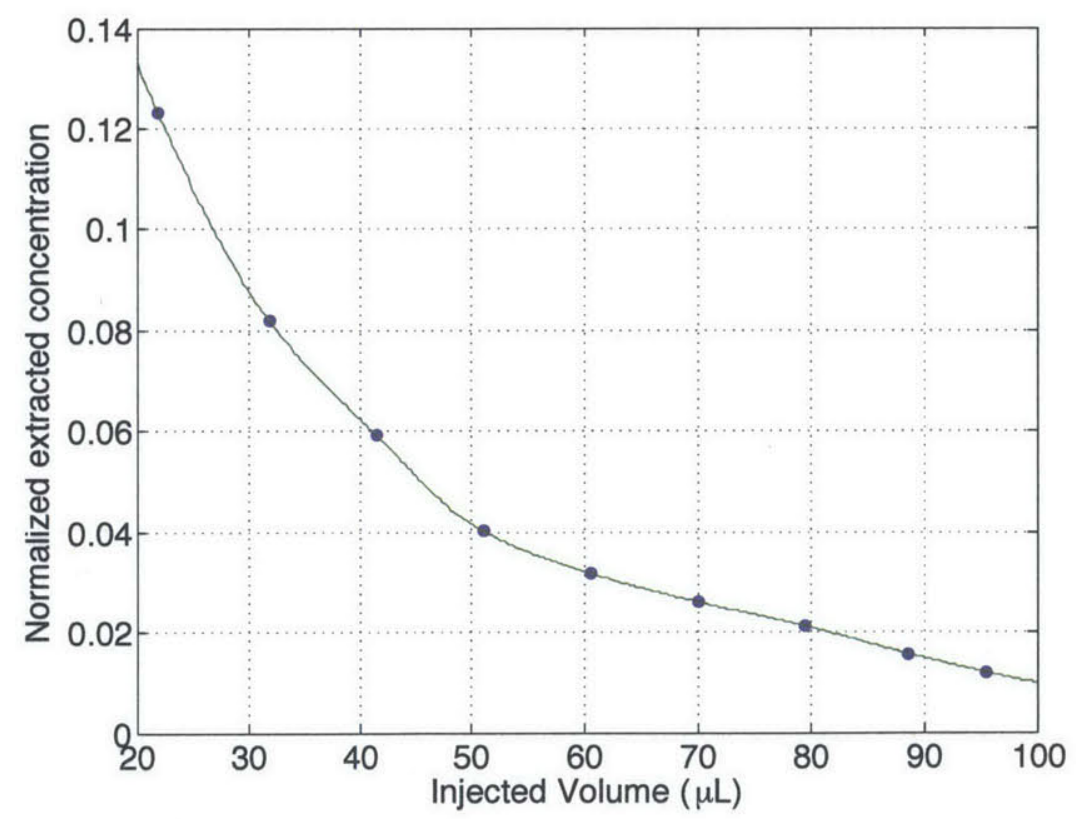

(b) Effect of injected volume on extracted concentration (normalized)

Figure 5-14: Results showing the effect of injection volume ( $\mathrm{Vol}$ ) on extracted volume and concentration. 


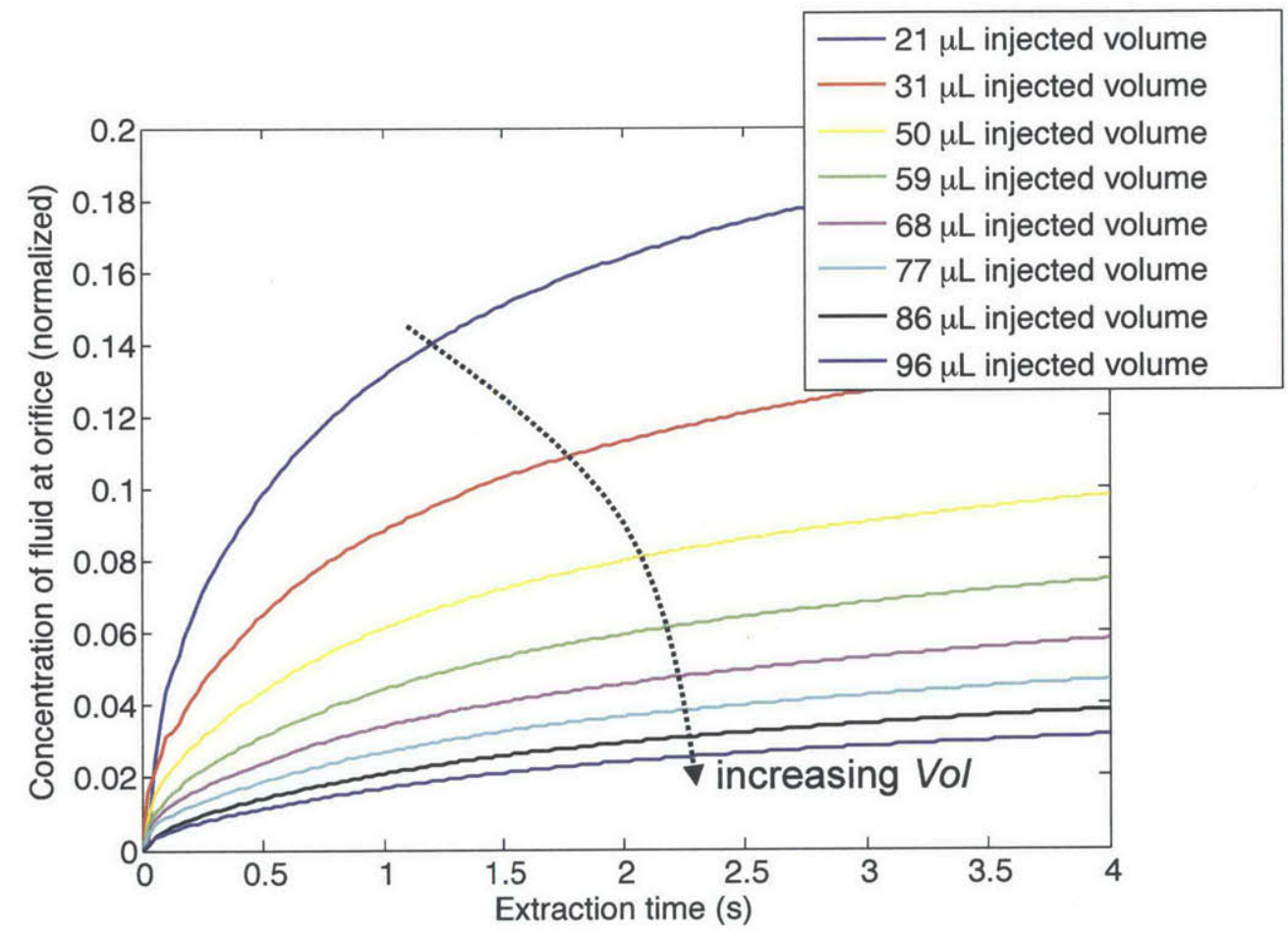

Figure 5-15: As the injected volume increases, the concentration of fluid at the orifice decreases. 
are shown in Figure 5-16.

Figure 5-16 shows that as the wait time is increased, the extracted volume decreases. Additionally, as the wait time is increased, the extracted concentration increases. This is because as the wait time is increased, the pressure burst in the tissue caused by injection dissipates, resulting in less fluid flow out of the puncture hole. However, there is also greater mixing between the injectate and ISF, resulting in higher extracted concentrations with longer wait times.

\section{Extraction speed}

Piston retraction speeds, or extraction speeds of $5 \mathrm{~mm} / \mathrm{s}, 10 \mathrm{~mm} / \mathrm{s}, 15 \mathrm{~mm} / \mathrm{s}, 20$ $\mathrm{mm} / \mathrm{s}, 25 \mathrm{~mm} / \mathrm{s}$, and $50 \mathrm{~mm} / \mathrm{s}$ were simulated for a $50 \mu \mathrm{L}$ injection performed with an injection pressure of $39.3 \mathrm{MPa}$ for $1.8 \mathrm{~s}$, and the results are shown in Figure 5-17. The simulations show that the extracted concentrations and volumes change little with a change in extraction speed.

However, we should note the changes in permeability in the tissue during the extraction (Figure 5-18). For high extraction speeds, the vacuum quickly causes tissue compression at the orifice, decreasing tissue permeability. Flow out of the puncture hole is therefore impeded much sooner than at lower extraction speeds. However, for slower extraction speeds, the pressure in the tissue caused by injection has dissipated to a greater extent over the course of the extraction, as more of the fluid has dispersed into the surrounding tissue rather than flowing back up through the puncture hole. There is therefore a trade-off between higher and lower extraction speeds: at higher extraction speeds there is a larger pressure differential between the tissue and the puncture hole as less of the injectate has dispersed into the surrounding tissue, but the higher extraction speed also causes the tissue at the orifice to compress much sooner, impeding fluid flow. 


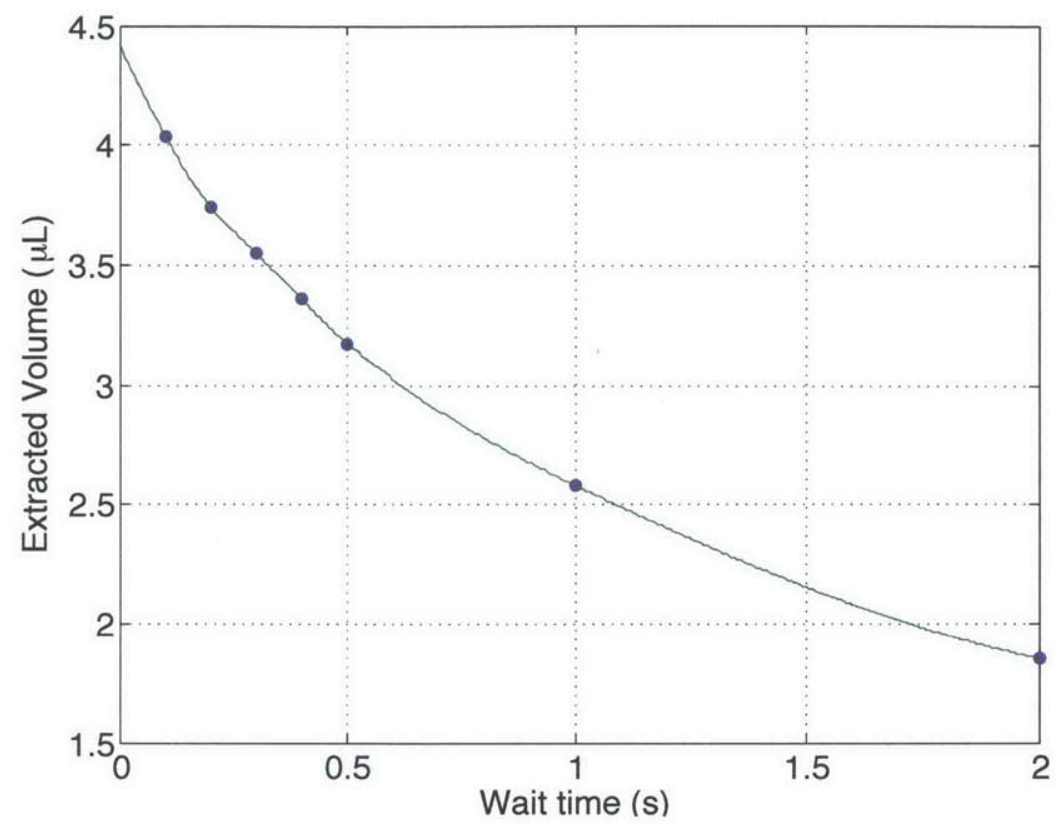

(a) Effect of wait time on extracted volume

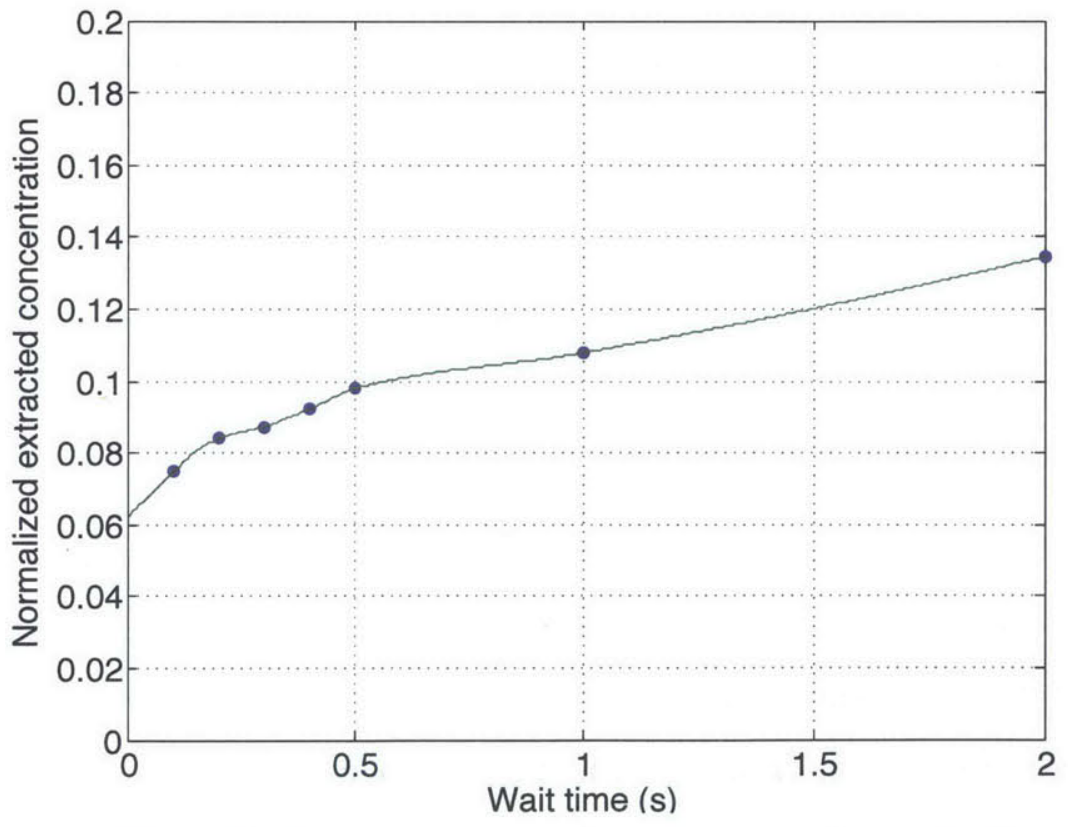

(b) Effect of wait time on extracted concentration (normalized)

Figure 5-16: Results showing the effect of wait time $\left(t_{\text {wait }}\right)$ on extracted volume and concentration. 


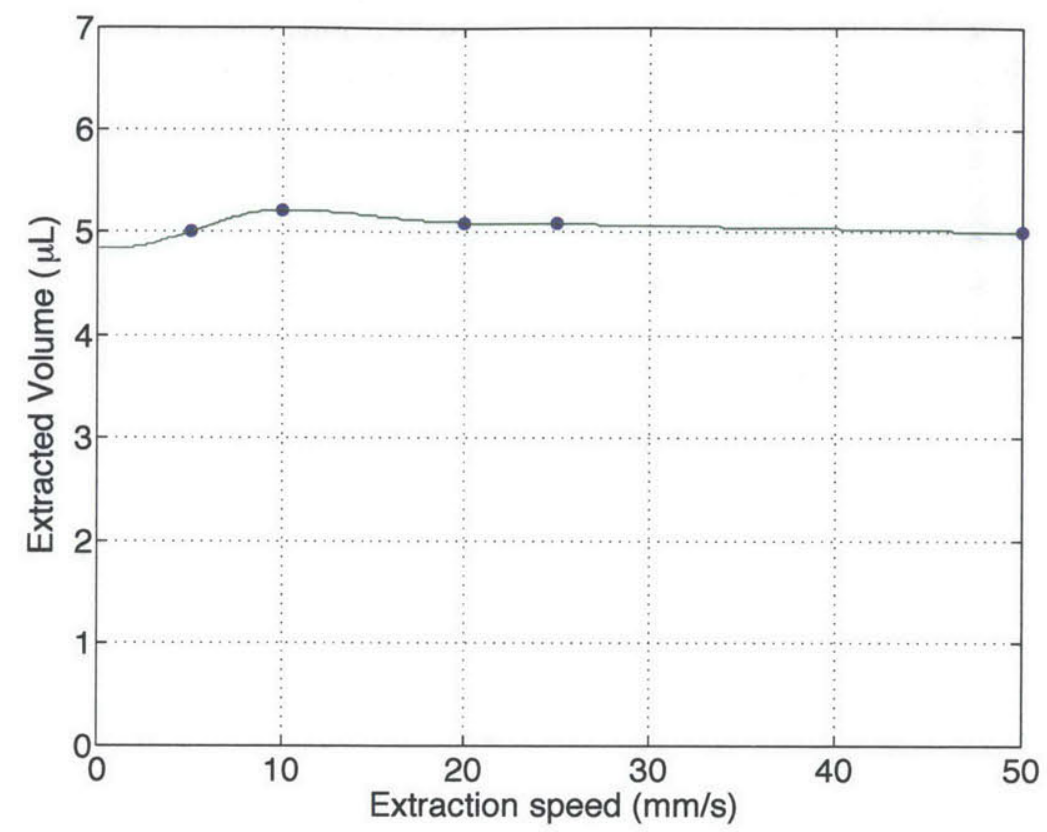

(a) Effect of extraction speed on extracted volumes.

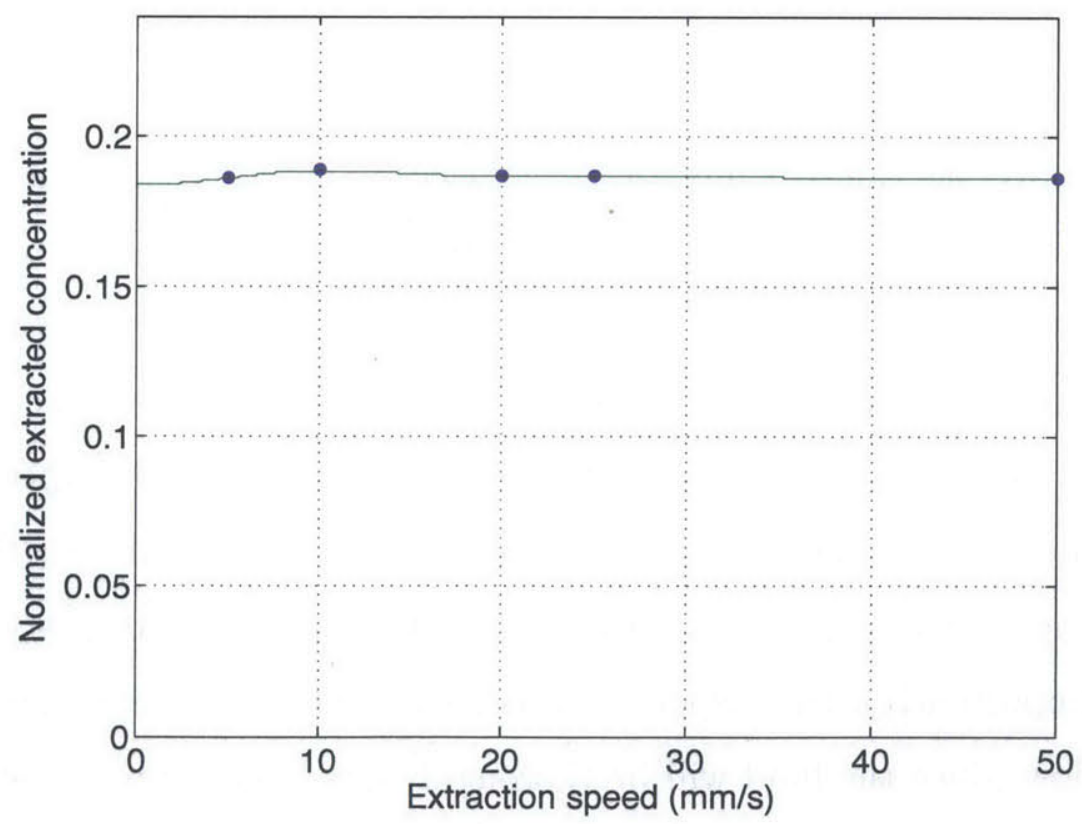

(b) Effect of extraction speed on extracted concentrations.

Figure 5-17: The effect of extraction speed ( $\left.v_{\text {piston }}\right)$ on extracted volume and concentration. 


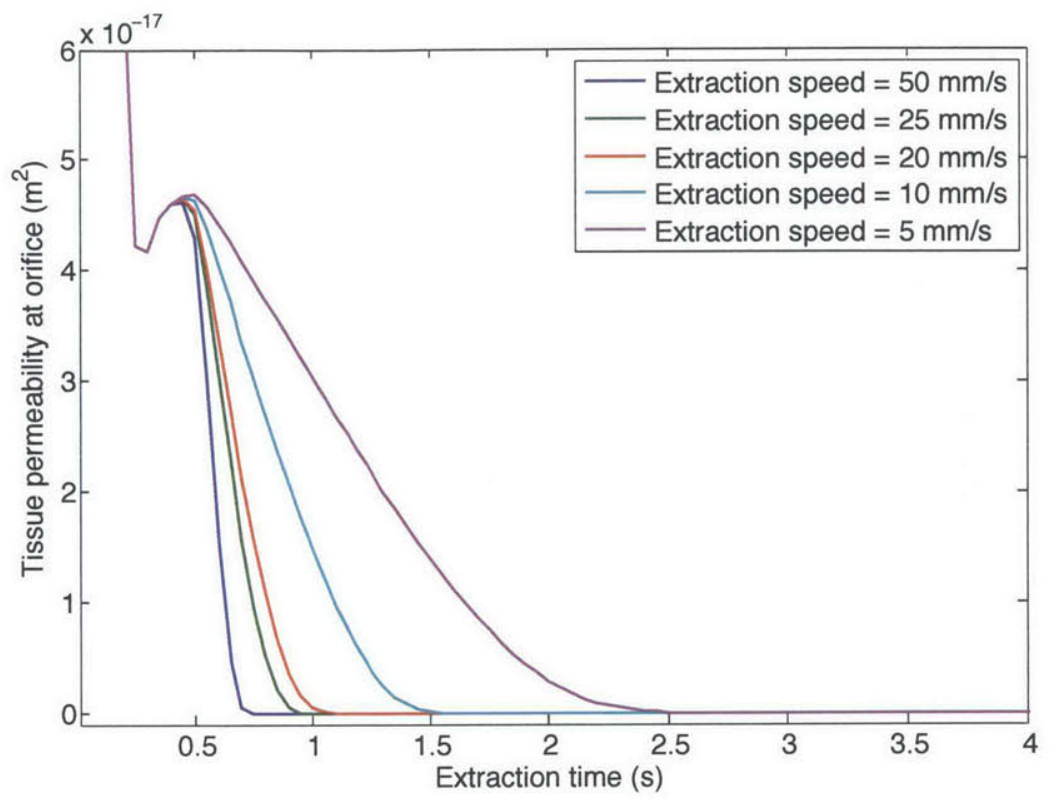

Figure 5-18: Tissue permeability at the orifice as a function of time and extraction speed.

\subsection{Discussion}

While the above-mentioned simulations modeled a simple needle injection prior to extraction, they offer important insights on how the injection and extraction parameters affect the resultant pressure and solute distributions in the tissue, and ultimately show how those parameters affect the extraction process. However, we can expect a few things to be different with needle-free injection. For one, it is expected that the injected fluid have turbulent behavior. This has several implications: first, Darcy's Law is no longer valid, and the Burke and Plummer equation becomes valid. Second (and more importantly), the dispersion coefficient will be different than in the case of laminar flow, since the fluid will be thoroughly mixed in the pore before it leaves the pore (diffusion is completely negligible). The dispersion coefficient is commonly described in terms of the Peclet number $(P e)$, and is given by:

$$
\alpha=\frac{d_{p}}{P e} .
$$


$P e$ is assumed to have different values for the longitudinal and transverse directions of flow. Theoretical studies have shown that the longitudinal Peclet number, $P e_{l}$, approaches a value of 2 while the transverse Peclet number, $P e_{t r}$, approaches a value of 11. Thus, in terms of solute transport, we can expect there to be greater mixing in the case of fluid extraction using a jet injector. With greater mixing, we can expect $t_{\text {wait }}$ to have less of a profound effect on extracted concentrations, as the injectate will mix with ISF on a much faster timescale than with a needle.

The model also does not account for the fracture mechanics of tissue. We can expect the breakage of collagen fibers to also have an effect on the extraction process. Air leakage (seal establishment) between the ampoule and tissue also was not considered in the model, and may be an important factor during experimentation.

It is also important to consider the possibility of the presence of gas bubbles in the tissue. It is possible that gas may be introduced during the injection phase. Orlob and Radhakrishna found that in an inhomogenous medium, there will be less dispersion if gas bubbles were entrapped in the larger pores, but no effect on dispersion if the gas bubbles were small (occupied less than $5 \%$ of the total pore volume) [73]. 


\section{Chapter 6}

\section{Extraction studies}

The purpose of the studies presented in this chapter is to prove the feasibility of the ISF acquisition method using the jet injector. Experiments were first performed on post-mortem porcine skin (which is most similar to human skin) to determine the effect of different parameters on the extraction process and make a comparison with the results generated by the model in Chapter 5. The lessons learned from both the model and the experimentation on post-mortem porcine skin were then applied to generate the injection and extraction parameters for a different animal model, the rat. Feasibility tests were then conducted on live rats to prove the capability of the jet injector in extracting ISF samples from live animals.

\subsection{Experiments on Post-Mortem Tissue: Porcine}

\subsubsection{Experimental Methods}

Post-mortem porcine tissue was obtained and prepared by the methods described in Section 3.2.3. Undiluted ISF samples were obtained by excising a portion of the dermis from the sample batch and centrifuging the fluid out of the tissue (Figure 6-1a). Surface contamination samples were also collected to ensure that the analytes detected in the extracted fluid samples were from within the tissue and not from the skin surface. Surface contamination samples were collected by placing $20 \mu \mathrm{L}$ of sterile 
saline on the surface of the skin of each tissue sample (Figure 6-1b).

For each extraction, the JI applied a contact force of $0.8 \mathrm{~N}$ onto the skin. The JI injected the tissue with a preset waveform (Figure 3-1), waited for a set period of time $\left(t_{\text {wait }}\right)$, and then extracted a mixture of the injectate and ISF (Figure 6-1c) with a preset extraction waveform (Figure 6-2). Injection and extraction parameters were tested to determine their effects on extracted volumes and concentrations. Protein and glucose concentrations of each collected sample were quantified using a NanoOrange ${ }^{\circledR}$ Protein Quantitation Kit (Life Science Technologies ${ }^{\top M}$ ) and an Amplex ${ }^{\circledR}$ Red Glucose/Glucose Oxidase Assay Kit (Life Science Technologies ${ }^{\top \mathrm{TM}}$ ). Acquired ISF sample concentrations were compared to surface contamination samples, and an extraction was deemed to be successful if the analyte concentration of the extracted sample was greater than the analyte concentration of the surface contamination sample. For comparison between trials, analyte concentrations were normalized by the respective protein and glucose concentrations in the undiluted ISF samples. The volumes of the extracted samples were also measured. Data sets were compared using the two tail $t$-test for unequal variances.
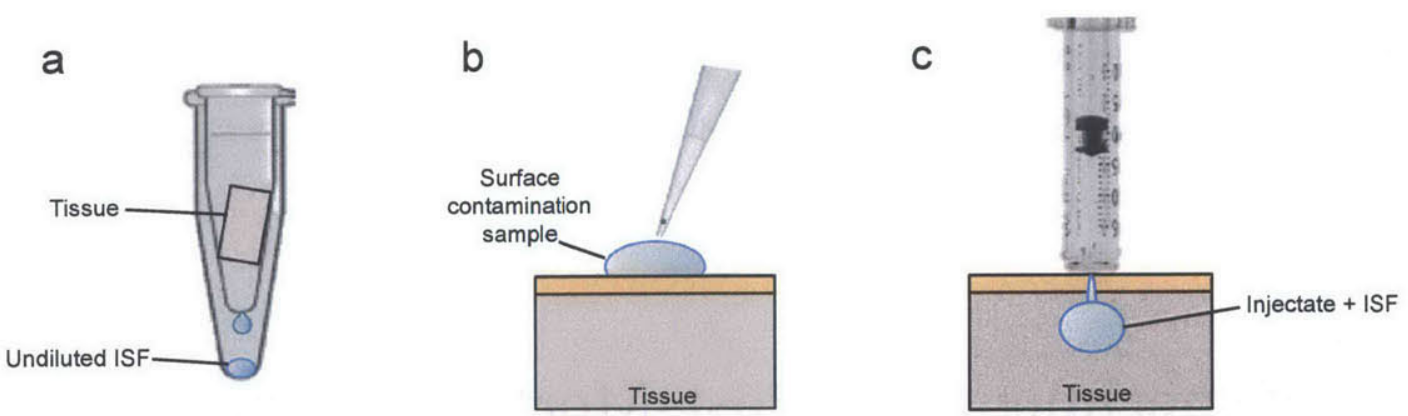

Figure 6-1: (a) Undiluted ISF obtained by centrifuging fluid out of a tissue sample; (b) Surface contamination sample collected by placing $20 \mu \mathrm{L}$ of sterile saline on the skin surface; (c) Extracted sample was a mixture of the injectate and ISF.

\subsubsection{Dependence on Injection Parameters}

For Trials A-D, the injection parameters $v_{j e t}$ and $V o l$ were varied while $t_{j e t}$ and $v_{\text {follow }}$ were unchanged $\left(t_{\text {jet }}=2 \mathrm{~ms}\right.$ and $\left.v_{\text {follow }}=50 \mathrm{~m} / \mathrm{s}\right)$. The extraction parameters were kept constant between trials; the piston was retracted using the single-phase 


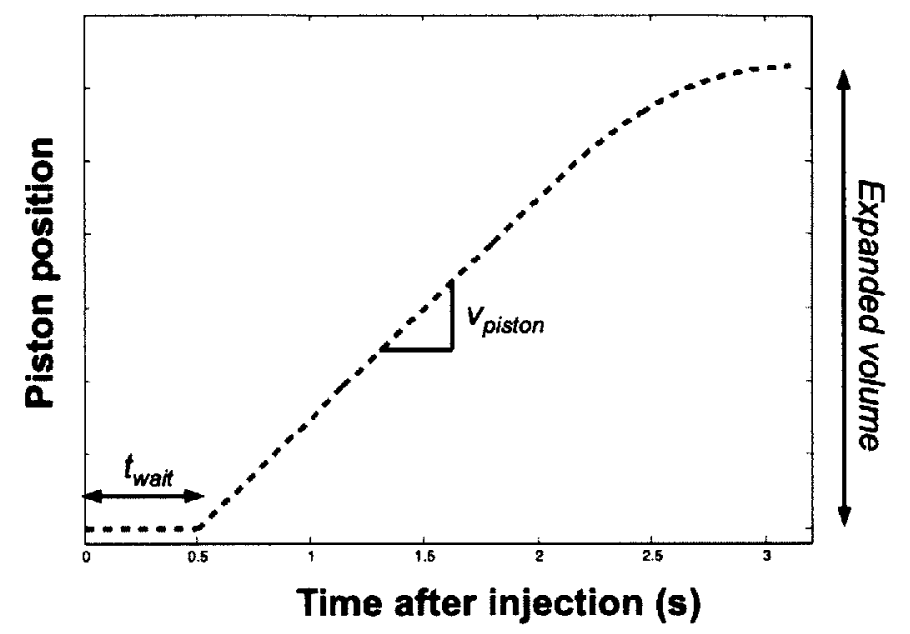

(a) Single-phase extraction waveform.

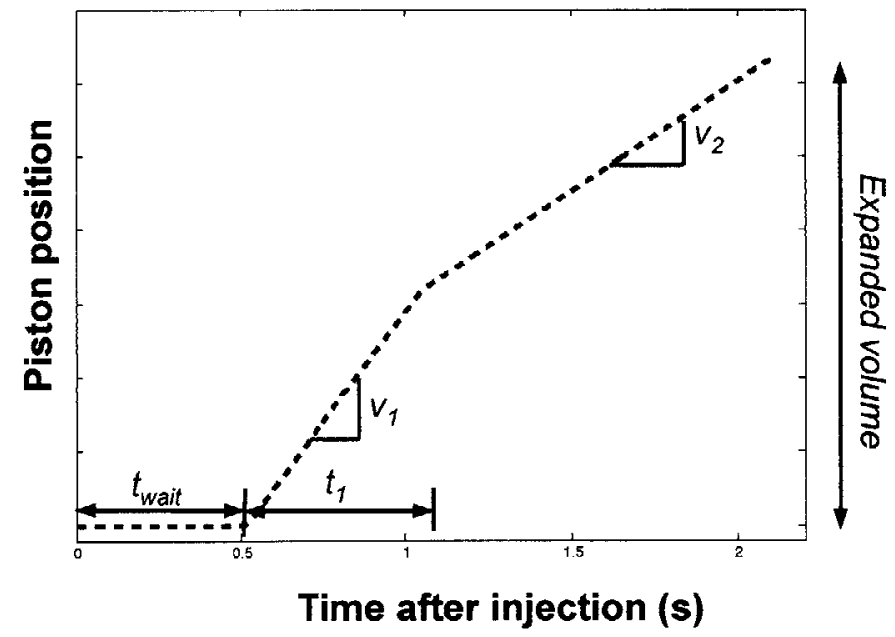

(b) Two-phase extraction waveform.

Figure 6-2: Extraction waveforms tested. 
waveform shown in Figure 6-2a with $v_{\text {piston }}=10 \mathrm{~mm} / \mathrm{s}$, to a travel length of $21 \mathrm{~mm}$ (corresponding to a volume expansion of $210 \mathrm{~mm}^{3}$ ), with a wait time of $400 \mathrm{~ms}$. The extracted volumes and concentrations were measured and the results are shown in Figure 6-3. A list of the injection parameters tested is shown in Table 6.1.

Table 6.1: Injection parameters

\begin{tabular}{|c|c|c|}
\hline Trial & $v_{\text {jet }}(\mathrm{m} / \mathrm{s})$ & Vol $(\mu \mathrm{L})$ \\
\hline \hline $\mathrm{A}$ & 150 & 50 \\
$\mathrm{~B}$ & 175 & 50 \\
$\mathrm{C}$ & 150 & 100 \\
$\mathrm{D}$ & 175 & 100 \\
\hline
\end{tabular}

Injecting with $50 \mu \mathrm{L}$ (Trials $\mathrm{A}$ and B) rather than $100 \mu \mathrm{L}$ (Trials C and D) yielded higher extracted protein and glucose concentrations due to less dilution from the injectate. This agrees with the model in Chapter 5, which saw higher extracted concentrations and lower extracted volumes from lower injection volumes. Injecting with $50 \mu \mathrm{L}$ also resulted in much lower variability in the extracted volumes.

There was no significant difference in extracted volumes and concentrations between Trials A $\left(v_{j e t}=150 \mathrm{~m} / \mathrm{s}\right)$ and B $\left(v_{j e t}=175 \mathrm{~m} / \mathrm{s}\right)$. This agrees with the FEM model, which saw only slight changes in extracted volumes and concentrations for different injection velocities. However, it has been previously reported that the magnitude of $v_{j e t}$ will establish the injection depth. In Chapter 3 , it was observed that the injectate stayed within the dermis and shallow subcutaneous layer for $50 \mu \mathrm{L}$ injections performed with $v_{j e t}=150 \mathrm{~m} / \mathrm{s}$, while the injectate reached deeper into the subcutaneous layer and even to the muscle when $v_{j e t}=175 \mathrm{~m} / \mathrm{s}$. A $v_{j e t}$ of $150 \mathrm{~m} / \mathrm{s}$ was deemed to be more appropriate than $v_{j e t}=175 \mathrm{~m} / \mathrm{s}$, since the target acquisition site is the dermis. It can thus be expected that the injection parameters of Trial A $\left(v_{j e t}=150 \mathrm{~m} / \mathrm{s}, t_{j e t}=2 \mathrm{~ms}, v_{\text {follow }}=50 \mathrm{~m} / \mathrm{s}\right.$, and $\left.\mathrm{Vol}=50 \mu \mathrm{L}\right)$ will be appropriate for human skin. 
a

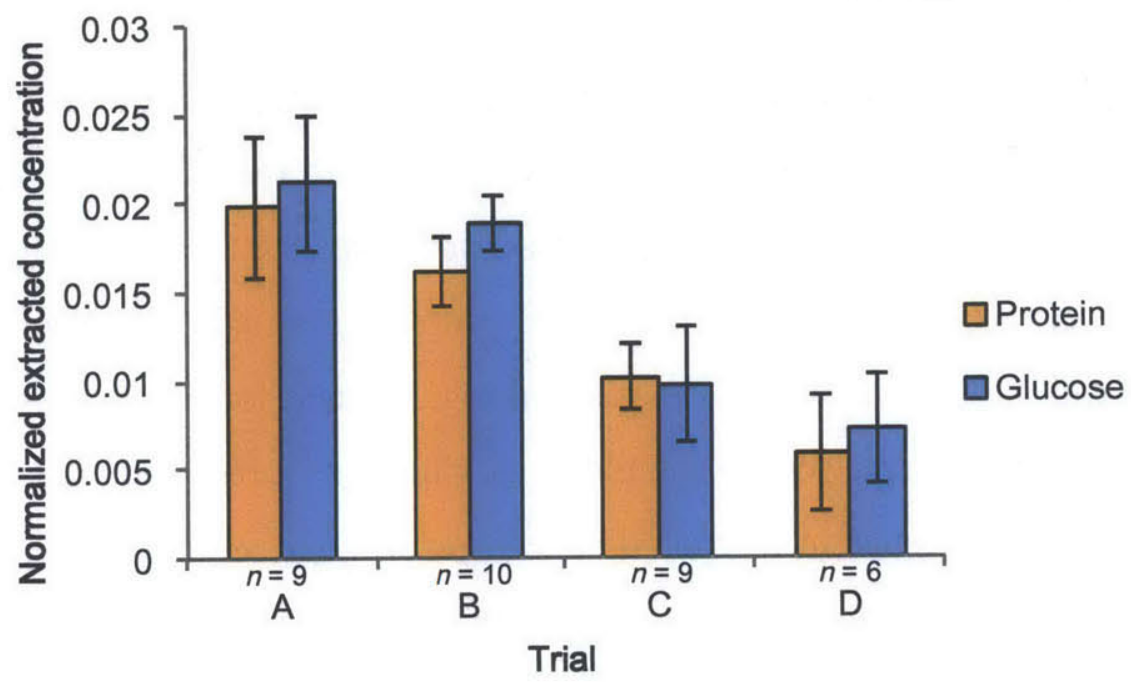

b

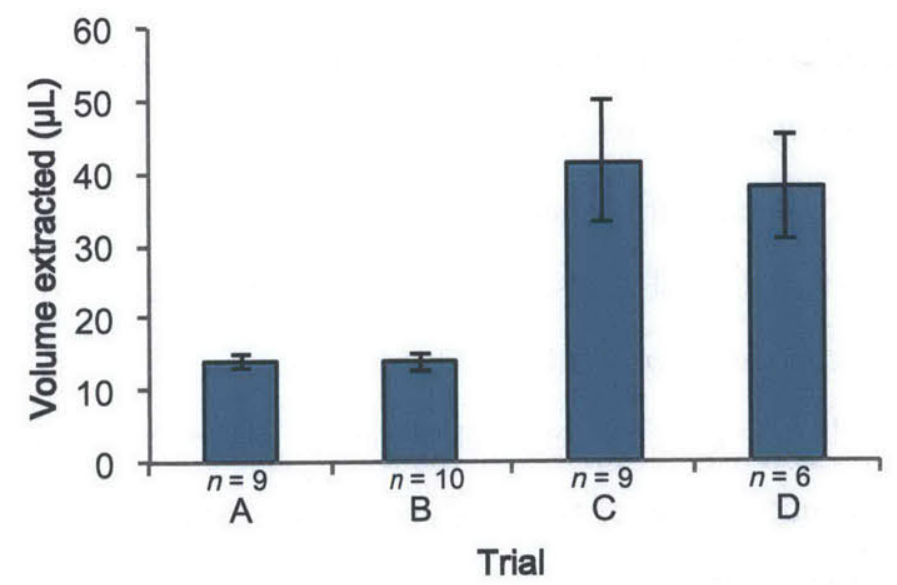

Figure 6-3: Results of trials testing different injection parameters. (a) Extracted protein and glucose concentrations (normalized by concentration in undiluted ISF) of samples. (b) Extracted volumes. Error bars in both plots represent standard errors. 


\subsubsection{Dependence on Extraction Parameters}

The injection parameters of Trial A $\left(v_{\text {jet }}=150 \mathrm{~m} / \mathrm{s}, t_{j e t}=2 \mathrm{~ms}, v_{\text {follow }}=50 \mathrm{~m} / \mathrm{s}\right.$, and $V o l=50 \mu \mathrm{L}$ ) were used for all subsequent trials.

\section{Single-phase extraction}

The single-phase extraction waveform shown in Figure 6-2a was initially chosen due to its simplicity. Different values of $t_{\text {wait }}$ and $v_{\text {piston }}$ were tested (Table 6.2). Results are shown in Figure 6-4. For the single-phase waveform, extracting with $v_{\text {piston }}=10$ $\mathrm{mm} / \mathrm{s}$ (Trial A) recovered significantly more protein $(p=0.05)$ than when $v_{p i s t o n}=$ $5 \mathrm{~mm} / \mathrm{s}$ (Trial E). There was no statistical significance for the recovered glucose concentrations or volumes. While the model in Chapter 5 predicted that there would be no significant difference in extracted concentrations and volumes with a change in extraction speed, the model did not account for air leakage between the ampoule and the skin surface. It is possible that the difference in extracted protein concentrations for the different $v_{\text {piston }}$ values may be from a seal being formed between the ampoule and skin surface faster for $v_{\text {piston }}=10 \mathrm{~mm} / \mathrm{s}$.

There was also no statistical difference in the extracted protein and glucose concentrations for a shorter $t_{\text {wait }}$ (Trial A vs. Trial F). A shorter $t_{\text {wait }}$, however, resulted in higher overall volume recovery $(p=0.03)$, which agrees with the model which predicts higher extracted volumes for shorter wait times. A shorter $t_{\text {wait }}$ is advantageous because although the concentrations of the analytes in the extracted sample remained unchanged between the two trials, the overall amount of analytes was greater for Trial $\mathrm{F}$ due to the larger recovered volumes. A short $t_{\text {wait }}$ is favorable for applications such as proteomic characterization, where the presence of certain biomarkers is of interest rather than the actual concentration of such analytes.

\section{Two-phase extraction}

The two-phase extraction waveform shown in Figure $6-2 \mathrm{~b}$ was then tested. It was hypothesized that this waveform shape would allow for better recovery since the initial 
Table 6.2: Single-phase extraction parameters

\begin{tabular}{|c|c|c|}
\hline Trial & $v_{\text {piston }}(\mathrm{mm} / \mathrm{s})$ & Wait time $(\mathrm{ms})$ \\
\hline \hline $\mathrm{A}$ & 10 & 400 \\
$\mathrm{E}$ & 5 & 400 \\
$\mathrm{~F}$ & 10 & 80 \\
\hline
\end{tabular}

a

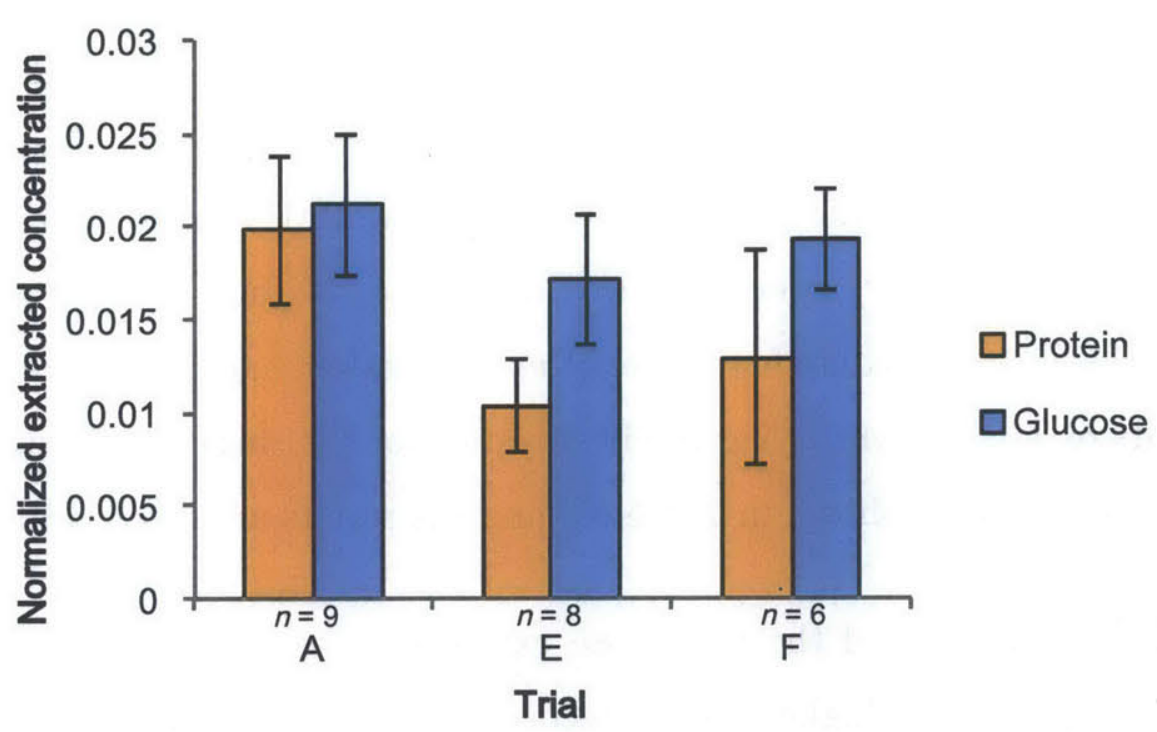

b

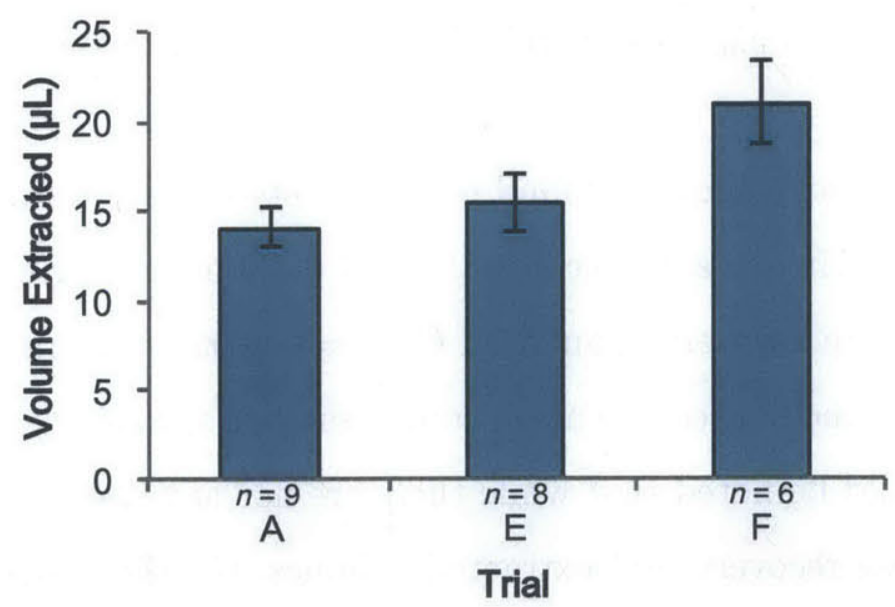

Figure 6-4: Results of trials testing different single-phase extraction parameters. (a) Extracted protein and glucose concentrations (normalized by concentration in undiluted ISF) of samples. (b) Extracted volumes. Error bars in both plots represent standard errors. 
Table 6.3: Extraction waveform parameters

\begin{tabular}{|c|c|c|}
\hline Trial & Extraction waveform type & Parameters \\
\hline \hline $\mathrm{F}$ & Single-phase & $v_{\text {piston }}=10 \mathrm{~mm} / \mathrm{s}$ \\
\hline $\mathrm{G}$ & Two-phase & $v_{1}=10 \mathrm{~mm} / \mathrm{s}$ \\
& & $t_{1}=1000 \mathrm{~ms}$ \\
& & $v_{2}=5 \mathrm{~mm} / \mathrm{s}$ \\
\hline $\mathrm{H}$ & Two-phase & $v_{1}=20 \mathrm{~mm} / \mathrm{s}$ \\
& & $t_{1}=500 \mathrm{~ms}$ \\
& & $v_{2}=5 \mathrm{~mm} / \mathrm{s}$ \\
\hline
\end{tabular}

high speed $\left(v_{1}\right)$ would quickly establish a seal between the tissue and the ampoule, and the slower secondary speed $\left(v_{2}\right)$ would slowly draw fluid into the ampoule with minimal clogging. Two different values of $v_{1}$ were tested, and results are compared to the results of Trial F, which had the best results for the single phase waveform. The parameters tested are shown in Table 6.3 and the results are shown in Figure 6-5.

Trial G, which tested the two-phase extraction waveform with $v_{1}=10 \mathrm{~mm} / \mathrm{s}$, resulted in significantly higher protein recovery than when extracting with the simple two-phase waveform with $v_{\text {piston }}=10 \mathrm{~mm} / \mathrm{s}$. We believe that this is because retracting the piston at $10 \mathrm{~mm} / \mathrm{s}$ serves to establish a seal quickly, and retracting at a slower secondary speed of $5 \mathrm{~mm} / \mathrm{s}$ allows for fluid to be collected into the ampoule with less tissue clogging. Retracting at a higher initial speed of $20 \mathrm{~mm} / \mathrm{s}$ (Trial $\mathrm{H}$ ) resulted in significantly less protein recovery than Trial $\mathrm{G}(p=0.0002)$, which may be because $20 \mathrm{~mm} / \mathrm{s}$ was too fast and caused too much compression of the tissue, resulting in less ISF recovery. It should be noted that while there are not any statistically significant trends seen in glucose recovery and extracted volumes, the differences between the trials for these metrics may have been too slight to be reflected in the data.

These post-mortem tissue experiments showed that ISF can be extracted in a much shorter period of time (the extraction time of Trial $G$ was $3.1 \mathrm{~s}$ ) than conventional ISF extraction methods (10-20 minutes). 

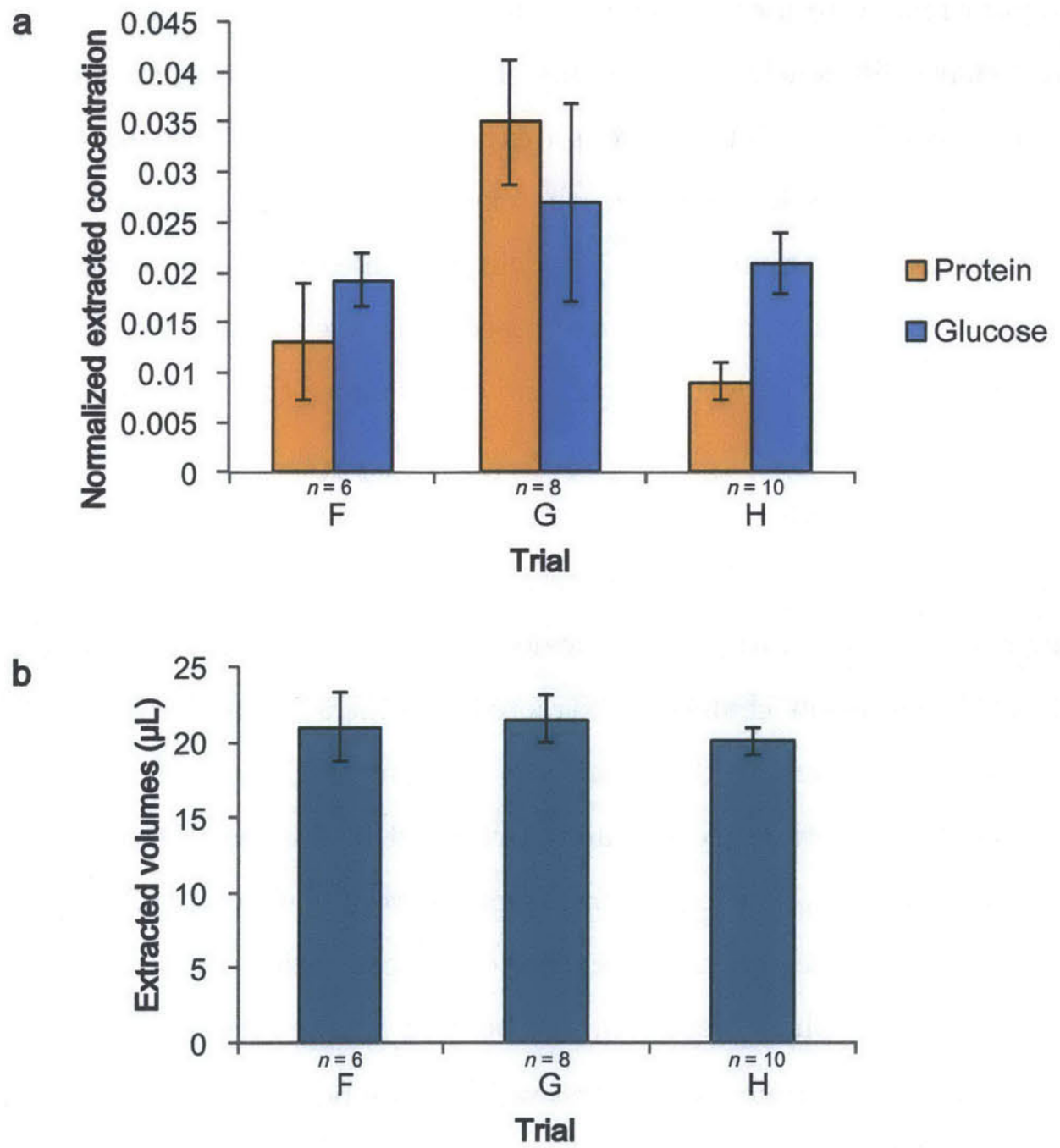

Figure 6-5: Results of trials testing extraction waveform shapes. (a) Extracted protein and glucose concentrations (normalized by concentration in undiluted ISF) of samples. (b) Extracted volumes. Error bars in both plots represent standard errors. 


\subsection{Lessons learned from modeling and experimen- tation}

Because the ISF acquisition method was shown to be feasible on post-mortem porcine skin, preparations were made to conduct a live animal study on rats. Since rat tissue may behave differently than porcine skin, the correct injection and extraction parameters needed to be found through experimentation on post-mortem rat skin.

The trends and lessons learned from the experimentation and modeling were used to prepare for the live animal study. Modeling revealed that as injection volume increased, the extracted concentration decreased while the extracted volume increased. Experimentation agreed with the model, and also revealed that high injection volumes resulted in high variability in extracted volumes. Thus, it is desirable to find the injection volume that yields consistent extracted volumes that are large enough for ease of handling, yet small enough for concentrations to be detectable by standard assay. Modeling and experimentation also revealed that increasing the injection velocity resulted in an insignificant change in extracted concentration and volume. We found that it is more important to determine the $v_{j e t}$ that targets the dermis. Increasing the time between injection and extraction resulted in decreased extracted volumes, and thus a minimal $t_{\text {wait }}$ is needed. Experimental results showed that the two-phase extraction waveform yielded higher extracted concentrations than the single-phase waveform, so the two-phase waveform will be used for the rat studies.

Injection depth experiments were first validated on post-mortem rat skin, and then the optimal two-phase extraction waveform parameters were determined. A handheld model was then constructed for use in the animal facility.

\subsubsection{Experiments with benchtop device on post-mortem rat skin}

The effect of injection parameters on the injection depth in rat skin has been studied extensively by Dr. Cathy Hogan, a research scientist in the BioInstrumentation 
Lab, and thus there was no need to conduct a comprehensive injection depth study. Instead, the injection parameters known to deliver to the dermis were validated to ensure that they yielded the same results with the new injection software. The injection parameters typically used to deliver to live rats with the legacy software was $v_{j e t}=130-140 \mathrm{~m} / \mathrm{s}, t_{j e t}=10 \mathrm{~ms}, v_{\text {follow }}=50 \mathrm{~m} / \mathrm{s}$, and $V o l=40-60 \mu \mathrm{L}$. Since the updated software changed the definition of $t_{j e t}$ slightly, $t_{j e t}=10 \mathrm{~ms}$ on the legacy software translated to $t_{j e t}=3 \mathrm{~ms}$ with $a c c=70 \mathrm{~m} / \mathrm{s}^{2}$ on the new software. The injection parameters of $v_{j e t}=130 \mathrm{~m} / \mathrm{s}, t_{j e t}=3 \mathrm{~ms}, v_{\text {follow }}=50 \mathrm{~m} / \mathrm{s}$ and $V o l=50$ $\mu \mathrm{L}$ were first tested on post-mortem rat skin. The injection depth was found to be $1.9 \pm 0.12 \mathrm{~mm}$ (standard error with $n=5$ ), and it was observed that the injectate did not reach the muscle. However, a small amount of splashback was also observed for some of the injections, which was undesirable since it meant that an inconsistent volume was being delivered across injections. Vol was lowered to a value of $40 \mu \mathrm{L}$, which yielded injection depths of $1.65 \pm 0.18 \mathrm{~mm}$ (standard error for $n=6$ ). Little to no splashback was observed, and the injectate did not reach the muscle. Thus, the injection parameters of $v_{j e t}=130 \mathrm{~m} / \mathrm{s}, t_{j e t}=3 \mathrm{~ms}, v_{\text {follow }}=50 \mathrm{~m} / \mathrm{s}$ and $V o l=40$ $\mu \mathrm{L}$ were used to validate needle-free extraction in post-mortem rat skin.

The extraction parameters were then tested on post-mortem rat skin. The twophase waveform was tested, since it was shown to have resulted in better extractions in the post-mortem porcine skin. The results, shown in Figure 6-6, show that there is an optimal value of $v_{1}$. Extracting with $v_{1}=20 \mathrm{~mm} / \mathrm{s}$ resulted in significantly higher extracted glucose concentrations than when extracting with $v_{1}=10 \mathrm{~mm} / \mathrm{s}(p$ $\left.=1.2 \times 10^{-8}\right)$ or $v_{1}=30 \mathrm{~mm} / \mathrm{s}(p=0.014)$. There was no significant difference in the extracted volumes across trials.

\subsubsection{Experiments with Handheld Device}

A handheld version of the JI was constructed for use in the animal lab (Figure 6-7). The handheld version did not include a force gauge to measure contact force, but a future version of the device may include a force gauge on the ampoule. The contact force was estimated by the user after practicing on post-mortem samples. 
a

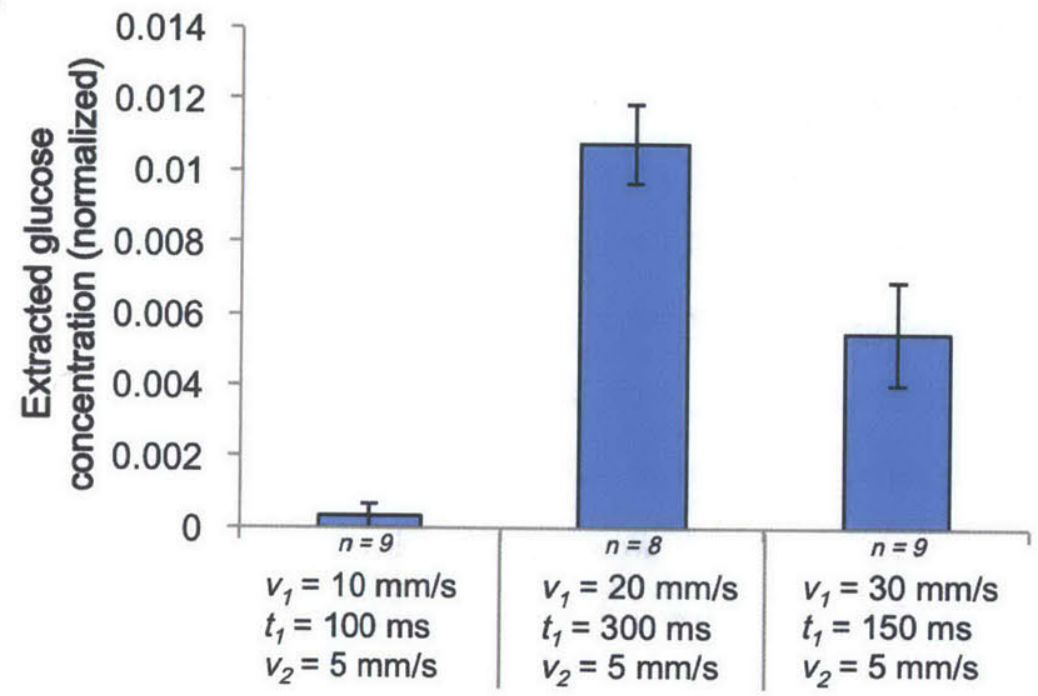

b

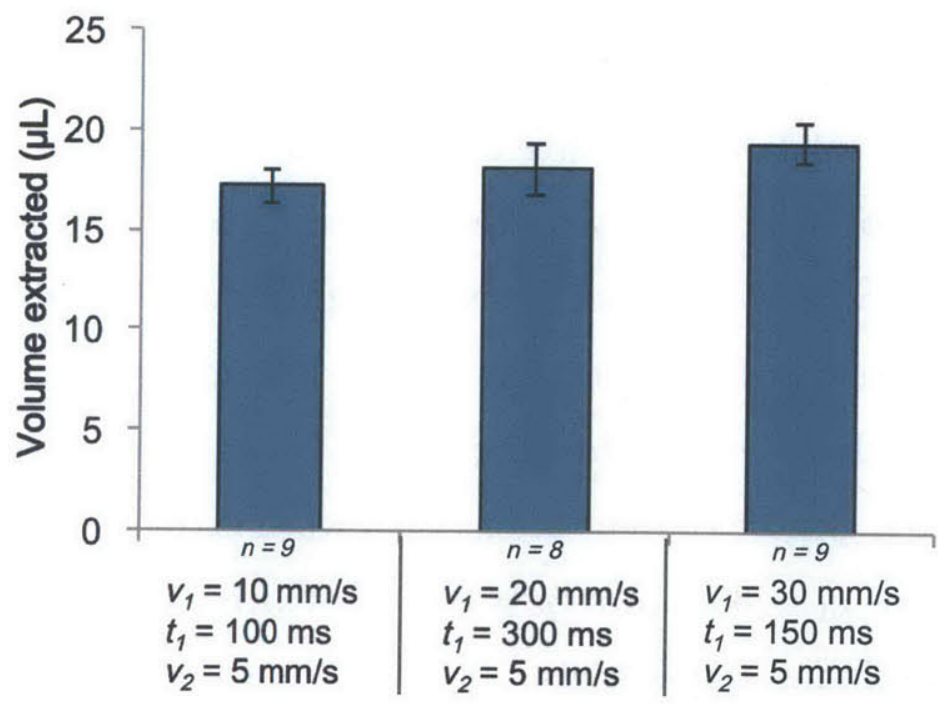

Figure 6-6: Results of experiments testing different values of $v_{1}$ on post-mortem rat skin. (a) Extracted glucose concentrations (normalized by concentration in undiluted ISF). (b) Extracted volumes. Error bars in both plots represent standard errors. 


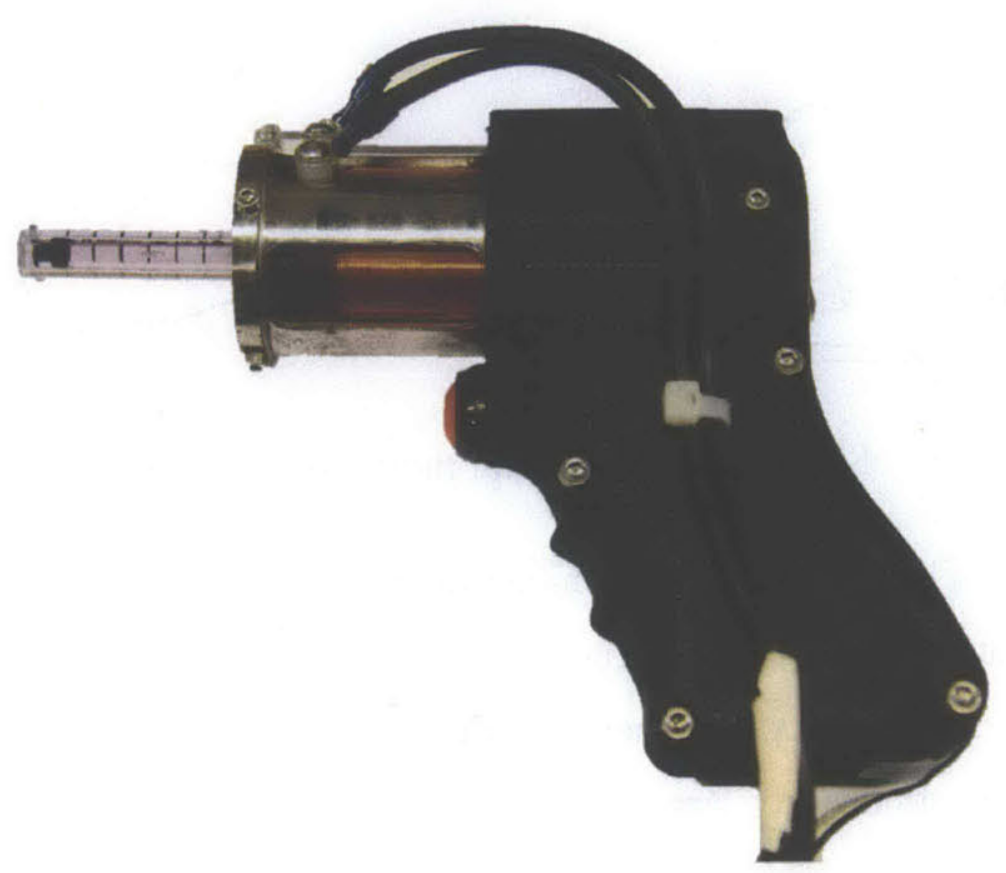

Figure 6-7: Handheld version of JI for live animal testing.

\subsection{Live animal studies: rat}

Five male rats were used to demonstrate the feasibility of the interstitial fluid acquisition method. Experiments were performed in the late afternoon. For each rat, blood glucose levels prior to interstitial fluid acquisition were measured by bleeding the tail vein and measuring the glucose level with a OneTouch ${ }^{\circledR}$ Ultra ${ }^{\circledR}$ Blue test strip and a OneTouch ${ }^{\circledR}$ Ultra ${ }^{\circledR} 2$ blood glucose monitoring system. Three sites were tested: the back of the neck, the left thigh, and the abdomen (Figure 6-8). Prior to fluid acquisition, each site was shaved and disinfected. A surface contamination sample was collected from each site by placing $20 \mu \mathrm{L}$ of sterile physiological saline on the acquisition site. Interstitial fluid was collected from each site with the following injection and extraction parameters: $v_{\text {jet }}=130 \mathrm{~m} / \mathrm{s}, t_{\text {jet }}=3 \mathrm{~ms}, v_{\text {follow }}=50 \mathrm{~m} / \mathrm{s}$, $t_{\text {wait }}=70 \mathrm{~ms}, v_{1}=20 \mathrm{~mm} / \mathrm{s}, t_{1}=300 \mathrm{~ms}$, and $v_{2}=5 \mathrm{~mm} / \mathrm{s}$. The volume of each extracted sample was measured and the glucose concentrations were measured using a BioVision Glucose Colorimetric/Fluorometric Assay Kit.

Since the injections were designed to target the dermis, blisters appeared on the skin after ISF extraction. Typical blister patterns for each extraction site are shown 

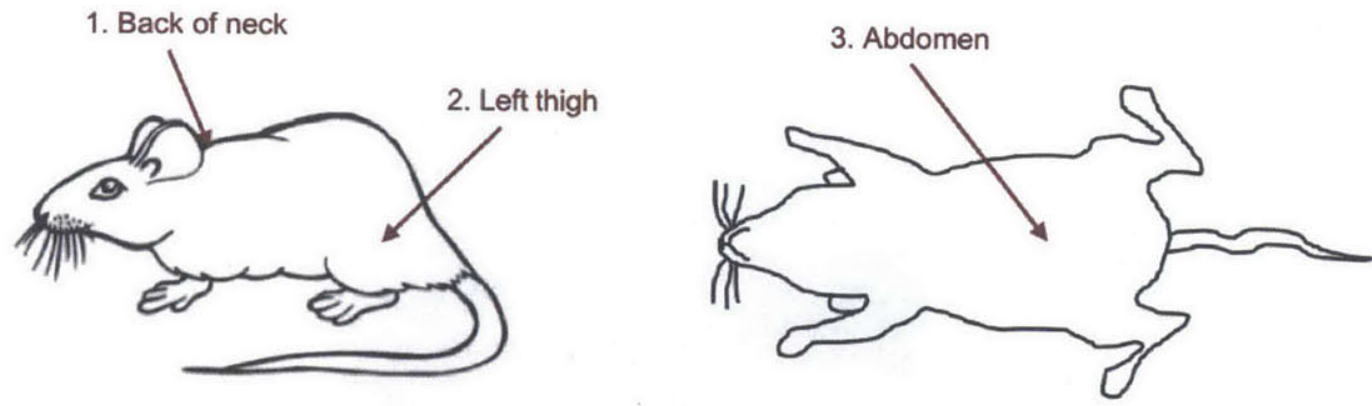

Figure 6-8: Interstitial fluid acquisition sites.

in Figure 6-9. The blisters were often elliptical in shape, which is in accordance with the anisotropic nature of skin. The lengths of the major and minor axes for each blister were measured and are shown in Figure 6-10. The blisters that appeared on the neck were significantly larger than the blisters that appeared on the thigh and abdomen ( $p=0.03$ and $p=0.05$, respectively). The back of the neck has a thicker layer of subcutaneous fat than in the thigh and abdomen, which may have contributed to the injected fluid spreading further in the lateral direction. A smaller blister may indicate that some injectate had been delivered into the muscle.

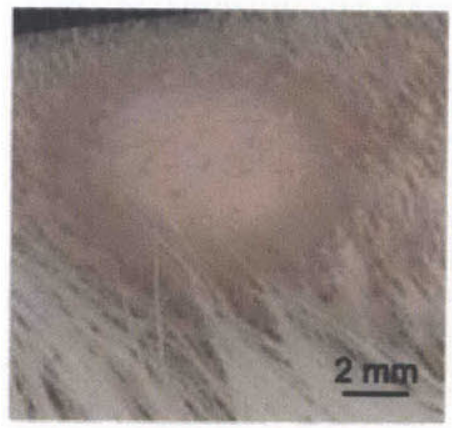

(a) Back of neck

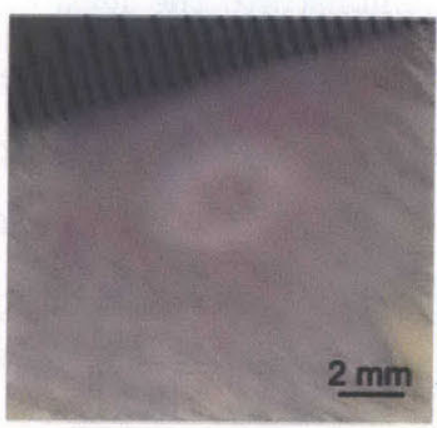

(b) Left thigh.

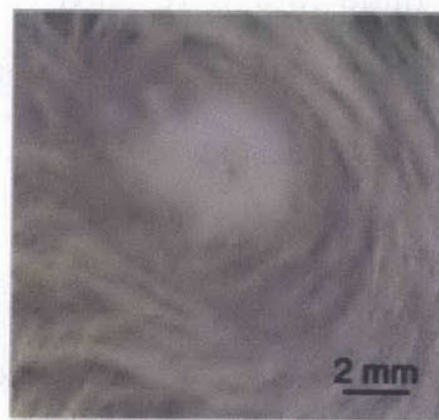

(c) Abdomen

Figure 6-9: Appearance of rat skin after interstitial fluid acquisition.

Samples extracted from the abdomen yielded the most consistent results, shown in Table 6.4. With the exception of Rat 3, who seemed to have a reaction to the isoflurane, the samples were diluted by a factor of 111-125. The dilution factor was calculated by comparing the glucose concentration in the extracted sample to the blood glucose level. It should be noted that while the dilution factors were fairly consistent, the extracted volumes were inconsistent and ranged from 8-30 $\mu \mathrm{L}$. 


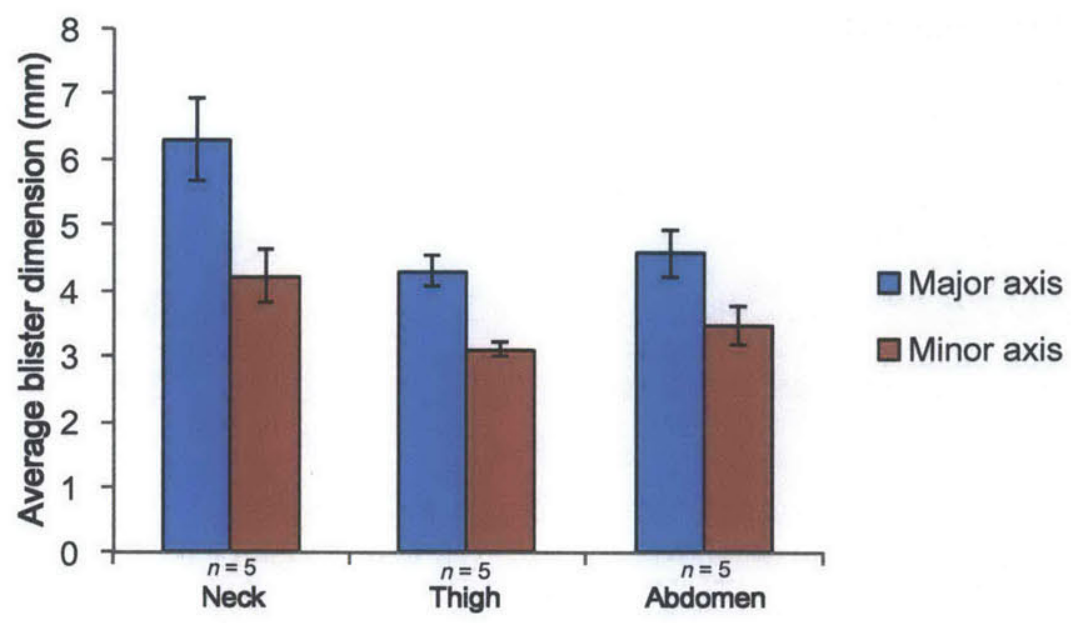

Figure 6-10: Average dimensions of the blisters formed due to ISF acquisition.

This can be attributed several reasons, such as the lack of a contact force sensor on the handheld device or variation among the rats. It is believed that the addition of a contact force sensor could greatly improve repeatability of extracted volumes. Measuring the mechanical properties of the skin prior to extraction and adjusting the injection and extraction parameters accordingly may also improve repeatability.

Table 6.4: Results from live rat study (samples extracted from the abdomen)

\begin{tabular}{|c|c|}
\hline Rat & Dilution factor \\
\hline \hline 1 & 125 \\
2 & 112 \\
3 & 201 \\
4 & 123 \\
5 & 111 \\
\hline
\end{tabular}

\subsection{Summary}

The effect of different injection and extraction parameters on the extraction process in post-mortem porcine skin was explored and compared to the model developed in Chapter 5. The lessons learned from the post-mortem skin experiments were used to apply the extraction process to rat skin. A live animal study on rats proved the 
feasibility of ISF extraction using the jet injector. 


\section{Chapter 7}

\section{Conclusions}

This thesis explored the effect of the jet injection process on tissue. The effect of contact force on delivery depth was investigated, and the role of $v_{\text {follow }}$ for injectate delivery was quantified with a microCT imaging study. Histology revealed that tissue damage caused by a jet injection was found to be remarkably different from the tissue damage caused by a needle injection. A new tool for the real-time visualization of jet injection into tissue - a high-speed X-ray imaging system - was built and characterized. For the first time, the jet dynamics in tissue were observed in real-time and quantified. The high-speed X-ray system allowed for the measurement of injectate speed within tissue, and revealed the dynamics of jet penetration. This information can be used to further optimize jet injections.

The second part of this thesis focused on developing the needle-less method of interstitial fluid sample acquisition using the jet injector. The jet injector software was overhauled and rewritten as a deterministic state machine for enhanced stability. The software was also designed to be modular such that different controllers/software schemes could easily be added for different applications. For this thesis, an extraction module was added. A finite element model of the extraction process was created in Comsol to elucidate the effect of different injection and extraction parameters on extracted concentrations and extracted volumes. Results from the injection experiments were used to set up the model. Experiments exploring the effect of injection and extraction parameters on ISF extraction from post-mortem porcine skin were 
performed and compared to the results from the model. Finally, the lessons learned from experimentation and modeling were used to determine the parameters for the live animal study. The feasibility of the extraction process was demonstrated on live rats.

\subsection{Suggestions for Future Work}

\subsubsection{Effect of jet injection on tissue}

The development of the high-speed X-ray imaging system opens the door for a whole host of new experiments. The jet dynamics in tissue of different injection profiles, such as a pulsing jet, can be explored. The dynamics of jet injection into live animals can also be investigated and compared to experiments on post-mortem tissue. The real-time images of jet injection into tissue can be compared to microCT volume reconstructions of the injectate dispersion pattern, generating a more complete understanding of the effect of different jet parameters on tissue. Furthermore, measurements of fluid velocity in tissue can be made and compared to the theoretical exit jet velocities.

\subsubsection{ISF acqusition}

\section{Jet injector device}

The jet injector device used in this thesis to demonstrate feasibility of the ISF acquisition process was a platform device to test the suitability of the jet injector for many different applications. Since it has been shown that the jet injector can be used for ISF acquisition, the device should be customized for this particular application. First, the device needs to be made portable. Since ISF acquisition only requires injection into the dermis, the size of the motor and power supply can be reduced (the

original motor was designed to reach the depths of the muscle). The ampoule should be redesigned to include a contact force sensor, as well as analytics to determine the components of the extracted ISF sample without having to remove the sample from 
the ampoule to perform separate assays.

\section{Future experiments}

Further work needs to be done to improve the repeatability of the acquisition process. It is believed that controlling the contact force may improve this. Tailoring the injection and extraction parameters for each individual may also greatly improve repeatability. This requires a module to measure the skin mechanics prior to extraction as well as experiments to investigate the effect of different skin mechanical properties on acquisition.

The different experimental techniques used in this thesis to understand the effect of jet injections on tissue (microCT imaging, high-speed X-ray imaging, histology) can be utilized to investigate the effect of the extraction process on tissue and assess whether further tissue damage is occurring due to extraction.

\section{Other applications}

This thesis focused on the development of the jet injector for dermal ISF acquisition. The jet injector can also be developed for the extraction of other fluids, such as tumor ISF, spinal fluid, and blood. The JI can also be developed to extract intact cells or even small tissue samples for the purpose of minimally invasive biopsies. The injection and extraction parameters required to collect these fluids need to be discovered through further modeling and experimentation. 


\section{Bibliography}

[1] "Ionlife water ionizer FAQs." http://www.ionizers.org/faq.html, Accessed 24 Oct 2013.

[2] "Necrotizing fasciitis." http://microbewiki.kenyon.edu/index.php/ Necrotizing_Fasciitis_(flesh\%_eating_bacteria_), Accessed 24 Oct 2013.

[3] I. A. Brown, "Scanning electron microscopy of human dermal fibrous tissue," Journal of Anatomy, vol. 113, pp. 159-168, November 1972.

[4] C. H. Daly, "Biomechanical properties of dermis," Journal of Investigative Dermatology, vol. 79, pp. 17-20, 071982.

[5] K. Comley and N. A. Fleck, "A micromechanical model for the Young's modulus of adipose tissue," International Journal of Solids and Structures, vol. 47, no. 21, pp. 2982-2990, 2010.

[6] "Geometric unsharpness calculations." www.ndt-ed.org/GeneralResources/ Formula/RTFormula/Unsharpness/GeometricUnsharpness.htm, Accessed 24 Oct 2013 .

[7] J. P. Bantle and W. Thomas, "Glucose measurement in patients with diabetes mellitus with dermal interstitial fluid," Journal of Laboratory and Clinical Medicine, vol. 130, no. 4, pp. 436 - 441, 1997.

[8] A. Machnik, A. Dahlmann, C. Kopp, J. Goss, H. Wagner, N. van Rooijen, K.-U. Eckardt, D. N. Müller, J.-K. Park, F. C. Luft, et al., "Mononuclear phagocyte system depletion blocks interstitial tonicity-responsive enhancer binding protein/vascular endothelial growth factor c expression and induces salt-sensitive hypertension in rats," Hypertension, vol. 55, no. 3, pp. 755-761, 2010.

[9] A.-M. Worm, "Exchange of macromolecules between plasma and skin interstitium in extensive skin disease," Journal of Investigative Dermatology, vol. 76, pp. 489-492, 061981.

[10] J. E. Celis, P. Gromov, T. Cabezón, J. M. A. Moreira, N. Ambartsumian, K. Sandelin, F. Rank, and I. Gromova, "Proteomic characterization of the interstitial fluid perfusing the breast tumor microenvironment," Molecular and Cellular Proteomics, vol. 3, no. 4, pp. 327-344, 2004. 
[11] R. K. Jain, "Transport of molecules in the tumor interstitium: A review," Cancer Research, vol. 47, no. 12, pp. 3039-3051, 1987.

[12] H. O. Fadnes, R. K. Reed, and K. Aukland, "Interstitial fluid pressure in rats measured with a modified wick technique," Microvascular Research, vol. 14, no. 1, pp. 27-36, 1977.

[13] U. Kiistala, "Suction blister device for separation of viable epidermis from dermis," Journal of Investigative Dermatology, vol. 50, no. 2, pp. 129-137, 1968.

[14] S. Mitragotri, "Current status and future prospects of needle-free liquid jet injectors," Nature Reviews Drug Discovery, pp. 543-548, 062006.

[15] P. D. Brinda, "Interstitial fluid sampler." Patent. U.S. 5682233, Oct 1997.

[16] T. P. Henning, R. G. Hiltibran, D. D. Cunningham, E. B. Shain, B. J. Tarkowski, and D. F. Young, "Method and apparatus for obtaining interstitial fluid for diagnostic tests." Patent. U.S. 6155992, Dec 2000.

[17] E. V. Mukerjee, S. D. Collins, R. R. Isseroff, and R. L. Smith, "Microneedle array for transdermal biological fluid extraction and in situ analysis," Sensors and Actuators A: Physical, vol. 114, no. 2-3, 2004.

[18] P. M. Wang, M. Cornwell, and M. R. Prausnitz, "Minimally invasive extraction of dermal interstitial fluid for glucose monitoring using microneedles," Diabetes Technology and Therapeutics, vol. 7, pp. 131-141, February 2005.

[19] W. Martanto, J. S. Moore, T. Couse, and M. R. Prausnitz, "Mechanism of fluid infusion during microneedle insertion and retraction," Journal of Controlled Release, vol. 112, pp. 357-361, 52006.

[20] A. Tezel, A. Sens, and S. Mitragotri, "A theoretical analysis of low-frequency sonophoresis: Dependence of transdermal transport pathways on frequency and energy density," Pharmaceutical Research, vol. 19, pp. 1841-1846, 2002.

[21] S. Mitragotri, M. Coleman, J. Kost, and R. Langer, "Transdermal extraction of analytes using low-frequency ultrasound," Pharmaceutical Research, vol. 17, pp. $466-470,2000$.

[22] J. Kost, S. Mitragotri, R. A. Gabbay, M. Pishko, and R. Langer, "Transdermal monitoring of glucose and other analytes using ultrasound," Nature Medicine, vol. 6 , no. 3 , pp. $347-350,2000$.

[23] S. Mitragotri, M. Coleman, J. Kost, and R. Langer, "Analysis of ultrasonically extracted interstitial fluid as a predictor of blood glucose levels," Journal of Applied Physiology, vol. 89, no. 3, pp. 961-966, 2000.

[24] "Dexcom G4 Platinum." http://www.dexcom.com/dexcom-g4-platinum, Accessed 24 Oct. 2013. 
[25] "Continuous glucose monitoring." http://www.medtronicdiabetes.com/ treatment-and-products/continuous-glucose-monitoring, Accessed 24 Oct 2013.

[26] E. Cengiz and W. V. Tamborlane, "A tale of two compartments: interstitial versus blood glucose monitoring," Diabetes Technology and Therapeutics, vol. 11, no. Supplement 1, pp. S-11-S-16, 2009.

[27] A. Taberner, N. C. Hogan, and I. W. Hunter, "Needle-free jet injection using realtime controlled linear Lorentz-force actuators," Medical Engineering 6 Physics, vol. 34, no. 9, pp. 1228-1235, 2012.

[28] J. E. White, J. H. Chang, N. C. Hogan, and I. W. Hunter, "Development of a Lorentz-force actuated intravitreal jet injector," Engineering in Medicine and Biology Society (EMBC), 2012 Annual International Conference of the IEEE, pp. 984-987, Aug. 28 2012-Sept. 12012.

[29] B. D. Hemond, D. M. Wendell, N. C. Hogan, A. J. Taberner, and I. W. Hunter, "A Lorentz-force actuated autoloading needle-free injector," Engineering in Medicine and Biology Society, 2006. EMBS '06. 28th Annual International Conference of the IEEE, pp. 679 682, Aug. 30 2006-Sept. 32006.

[30] D. M. Wendell, B. D. Hemond, N. C. Hogan, A. J. Taberner, and I. W. Hunter, "The effect of jet parameters on jet injection," in Engineering in Medicine and Biology Society. 28th Annual International Conference of the IEEE., 2006.

[31] J. Schramm-Baxter, J. Katrencik, and S. Mitragotri, "Jet injection into polyacrylamide gels: investigation of jet injection mechanics," Journal of Biomechanics, vol. 37 , no. 8, pp. 1181-1188, 2004.

[32] B. D. Hemond, A. Taberner, C. Hogan, B. Crane, and I. W. Hunter, "Development and performance of a controllable autoloading needle-free jet injector," Journal of Medical Devices, vol. 5, 2011.

[33] J. C. Stachowiak, T. H. Li, A. Arora, S. Mitragotri, and D. A. Fletcher, "Dynamic control of needle-free jet injection," Journal of Controlled Release, vol. 135, no. 2, pp. 104-112, 2009.

[34] R. M. Lavker, P. Zheng, and G. Dong, "Aged skin: a study by light, transmission electron, and scanning electron microscopy," Journal of investigative dermatology, vol. 88, pp. 44-51, 1987.

[35] D. Holland, R. Booy, F. D. Looze, P. Eizenberg, J. McDonald, J. Karrasch, M. McKeirnan, H. Salem, G. Mills, J. Reid, F. Weber, and M. Saville, "Intradermal influenza vaccine administered using a new microinjection system produces superior immunogenicity in elderly adults: a randomized controlled trial," Journal of Infectious Diseases, vol. 198, pp. 650-658, 2008. 
[36] E. A. Henderson, T. J. Louie, K. Ramotar, D. Ledgerwood, K. M. Hope, and A. Kennedy, "Comparison of higher-dose intradermal hepatitis $\mathrm{B}$ vaccination to standard intramuscular vaccination of healthcare workers," Infection Control and Hospital Epidemiology, vol. 21, no. 4, pp. 264-269, 2000.

[37] G. Kersten and H. Hirschberg, "Needle-free vaccine delivery," Expert Opinion on Drug Delivery, vol. 4, pp. 459-474, 2013/04/18 2007.

[38] J. C. Stachowiak, M. G. von Muhlen, T. H. Li, L. Jalilian, S. H. Parekh, and D. A. Fletcher, "Piezoelectric control of needle-free transdermal drug delivery," Journal of Controlled Release, vol. 124, no. 1-2, pp. 88-97, 2007.

[39] J. Schramm-Baxter and S. Mitragotri, "Needle-free jet injections: dependence of jet penetration and dispersion in the skin on jet power," Journal of Controlled Release, vol. 97, no. 3, pp. 527-535, 2004.

[40] J. Baxter and S. Mitragotri, "Jet-induced skin puncture and its impact on needlefree jet injections: Experimental studies and a predictive model," Journal of Controlled Release, vol. 106, no. 3, pp. 361-373, 2005.

[41] O. A. Shergold and N. A. Fleck, "Mechanisms of deep penetration of soft solids, with application to the injection and wounding of skin," Proceedings of the Royal Society of London. Series A: Mathematical, Physical and Engineering Sciences, vol. 460, no. 2050, pp. 3037-3058, 2004.

[42] J. Ankersen, A. Birkbeck, R. Thomson, and P. Vanezis, "Puncture resistance and tensile strength of skin simulants," Proceedings of the Institution of Mechanical Engineers, Part H: Journal of Engineering in Medicine, vol. 213, no. 6, pp. 493501, 1999.

[43] F. Figge and D. Barnett, "Anatomic evaluation of a jet injection instrument designed to minimize pain and inconvenience of parenteral therapy.," American practitioner and digest of treatment, vol. 3, no. 4, p. 197, 1948.

[44] K. Deisseroth and K. Chung, "Structural and molecular interrogation of intact biological systems," Nature, vol. 497, no. 7449, pp. 332-337, 2013.

[45] M.-T. Ke, S. Fujimoto, and T. Imai, "Seedb: a simple and morphology-preserving optical clearing agent for neuronal circuit reconstruction," Nature neuroscience, vol. 16, no. 8, pp. 1154-1161, 2013.

[46] E. L. Brainerd, D. B. Baier, S. M. Gatesy, T. L. Hedrick, K. A. Metzger, S. L. Gilbert, and J. J. Crisco, "X-ray reconstruction of moving morphology (XROMM): precision, accuracy and applications in comparative biomechanics research," Journal of Experimental Zoology Part A: Ecological Genetics and Physiology, vol. 313A, no. 5, pp. 262-279, 2010.

[47] "X-ray reconstruction of moving morphology: Hardware.." http://www.xromm. org/hardware, Accessed 24 Oct 2013. 
[48] J. Brayanov, "Design and development of a 3D X-ray microscope," Master's thesis, Massachusetts Institute of Technology, 2006.

[49] H. Darcy, Les fontaines publiques de la ville de Dijon. 1856.

[50] M. Rhodes, Introduction to Particle Technology. Wiley, 1989.

[51] J. R. Levick, "Flow through interstitium and other fibrous matrices," Experimental Physiology, vol. 72, no. 4, pp. 409-437, 1987.

[52] F. H. Silver, J. W. Freeman, and D. DeVore, "Viscoelastic properties of human skin and processed dermis," Skin Research and Technology, vol. 7, no. 1, pp. 18$23,2001$.

[53] L. T. Smith, K. A. Holbrook, and P. H. Byers, "Structure of the dermal matrix during development and in the adult," Journal of Investigative Dermatology, vol. 79, pp. 93-104, 071982.

[54] A. Maroudas, "Fluid transport in cartilage," Ann. Rheum. Dis, vol. 34, no. Suppl 2, p. 77, 1975.

[55] A. J. Grodzinsky, "Electromechanical and physicochemical properties of connective tissue.," Critical reviews in biomedical engineering, vol. 9, no. 2, p. 133, 1983.

[56] P. C. Carman, "Fluid Flow Through Granular Beds," Transactions - Institution of Chemical Engineeres, vol. 15, pp. 150-166, 1937.

[57] J. Happel and H. Brenner, Low Reynolds number hydrodynamics: with special applications to particulate media. Hingham, MA, U.S.A.: Kluwer Boston, 1983.

[58] M. Grabowska, "Collagen content of normal connective tissue, of tissue surrounding a tumour and of growing rat sarcoma," Nature, vol. 183, no. 4669, pp. 1186-1187, 1959.

[59] R. H. Pearce and T. C. Laurent, "Exclusion of dextrans by meshworks of collagenous fibres," Biochemical Journal, vol. 163, no. 3, pp. 617-625, 1977.

[60] C. A. Wiederhielm, "Dynamics of capillary fluid exchange: A nonlinear computer simulation," Microvascular Research, vol. 18, pp. 48-82, 71979.

[61] J. Bert and R. Pearce, "The interstitium and microvascular exchange," Handbook of Physiology, vol. 4, pp. 521-547, 1984.

[62] C. R. Ethier, "Hydrodynamics of flow through gels with applications to the eye," Master's thesis, Massachusetts Institute of Technology, 1983.

[63] B. N. Preston, M. Davies, and A. G. Ogston, "The composition and physicochemical properties of hyaluronic acids prepared from ox synovial fluid and from a case of mesothelioma," Biochemical Journal, vol. 96, pp. 449-474, 1965. 
[64] A. Maroudas, "Distribution and diffusion of solutes in articular cartilage," Biophysical Journal, vol. 10, no. 5, pp. 365-379, 1970.

[65] C. C. Michel, "The malpighi lecture. vascular permeability-the consequence of Malpighi's hypothesis," International Journal of Microcirculation: Clinical and Experimental, vol. 4, no. 3, pp. 265-284, 1985.

[66] J. L. Bert and R. K. Reed, "Flow conductivity of rat dermis is determined by hydration," Biorheology, vol. 32, no. 1, pp. 17-27, 1995.

[67] C. Edwards and R. Marks, "Evaluation of biomechanical properties of human skin.," Clinics in Dermatology, vol. 13, pp. 375-380, Jul-Aug 1995.

[68] P. Raimondi, G. H. F. Gardner, and C. B. Petrick, "Effect of pore structure and molecular diffusion on the mixing of miscible liquids flowing in porous media," preprint, vol. 43, pp. 6-9, 1959.

[69] R. Aris and N. R. Amundson, "Some remarks on longitudinal mixing or diffusion in fixed beds," AIChE Journal, vol. 3, no. 2, pp. 280-282, 1957.

[70] J. J. Carberry, "Axial dispersion and void-cell mixing efficiency in fluid flow in fixed beds," AIChE Journal, vol. 4, no. 1, pp. 13M-22M, 1958.

[71] J. Prausnitz, "Longitudinal dispersion in a packed bed," AIChE Journal, vol. 4, no. 1, pp. 14M-22M, 1958.

[72] T. K. Perkins and O. C. Johnston, "A review of diffusion and dispersion in porous media," SPE Journal, vol. 3, no. 1, pp. 70-84, 1963.

[73] G. T. Orlob and G. Radhakrishna, "The effects of entrapped gases on the hydraulic characteristics of porous media," Transactions, American Geophysical Union, vol. 39, pp. 648-659, 1958. 